\title{
The Italian National Food Consumption Survey INRAN-SCAI 2005-06: main results in terms of food consumption
}

\author{
Catherine Leclercq*, Davide Arcella, Raffaela Piccinelli, Stefania Sette, Cinzia Le Donne \\ and Aida Turrini on behalf of the INRAN-SCAI 2005-06 Study Groupt \\ INRAN, National Research Institute for Food and Nutrition, Via Ardeatina 546, I-00178 Rome, Italy
}

Submitted 25 January 2008: Accepted 18 December 2008: First published online 12 March 2009

\begin{abstract}
Objective: The current paper aims to present the main results of the Italian National Food Consumption Survey INRAN-SCAI 2005-06.

Design: A cross-sectional study was performed. Households were randomly selected after geographical stratification of the national territory. Food consumption was assessed on three consecutive days through individual estimated dietary records.

Setting: Italy.

Subjects: The final study sample comprised 3323 subjects (1501 males and 1822 females) aged $0 \cdot 1$ to $97 \cdot 7$ years belonging to 1329 households: fifty-two infants (0-2.9 years), 193 children (3-9.9 years), 247 teenagers (10-17.9 years), 2313 adults (18-64.9 years) and 518 elderly (65 years and above).

Results: Participation rate was $33 \%$. The mean ratio of estimated energy intake to estimated BMR was 1.41 in adults. Indicators of mean and high individual consumption are presented for fifteen large categories and fifty-one subcategories of foods and beverages, in the total population and in consumers, by age and sex categories. The overall consumption of fruit and vegetables was $418 \mathrm{~g} / \mathrm{d}$. The consumption of red meat was approximately $700 \mathrm{~g} /$ week, expressed as raw weight. Some specific aspects of the Italian food consumption pattern were confirmed: a large contribution from bread, pasta and pizza to cereals, from olive oil to fats and from wine to alcoholic beverages.

Conclusions: The database obtained from the survey will be the key reference for Italian food consumption during the coming years and will be utilized for a variety of purposes including the assessment of nutrient intakes and risk analysis.
\end{abstract}

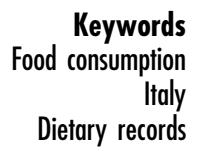

Food consumption patterns are changing rapidly in the Italian population. Important factors of change are the evolution of lifestyle, the availability of a large variety of new intensively advertised food products and the progressive ageing of the population. A steady increase of meals consumed away from home and of convenience foods has been observed ${ }^{(1)}$. The traditional Mediterranean diet, rich in plant foods, is being modified ${ }^{(2)}$.

The availability of data collected at individual level in various sections of the population is crucial to characterize food consumption patterns. These data are needed to perform a number of research and surveillance activities in the area of consumer science, nutrition and food safety. Nationwide Italian food consumption surveys had

† INRAN-SCAI 2005-06 Study Group: Davide Arcella, Noemi Bevilacqua, Marisa Capriotti, Giovina Catasta, Laura D'Addezio, Guglielmo Di Lena, Marika Ferrari, Catherine Leclercq, Cinzia Le Donne, Simona Martines, Lorenza Mistura, Antonella Pettinelli, Raffaela Piccinelli, Romana Roccaldo, Stefania Sette, Fulvia Spadoni, Elisabetta Toti and Aida Turrini. been performed in 1980-84 $4^{(3)}$ and $1994-96^{(4)}$. The Italian Ministry of Agriculture funded the third national food consumption survey, named INRAN-SCAI 2005-06, to update current dietary information.

The current paper aims to present the main results of the Italian National Food Consumption Survey INRANSCAI 2005-06.

\section{Methods}

A cross-sectional survey was conducted on a random sample of the Italian population.

\section{Sample}

A target sample of 1300 households was considered with the aim of characterizing average food consumption in the four main geographical areas of Italy (North-West, North-East, Centre and South and Islands). The Census 


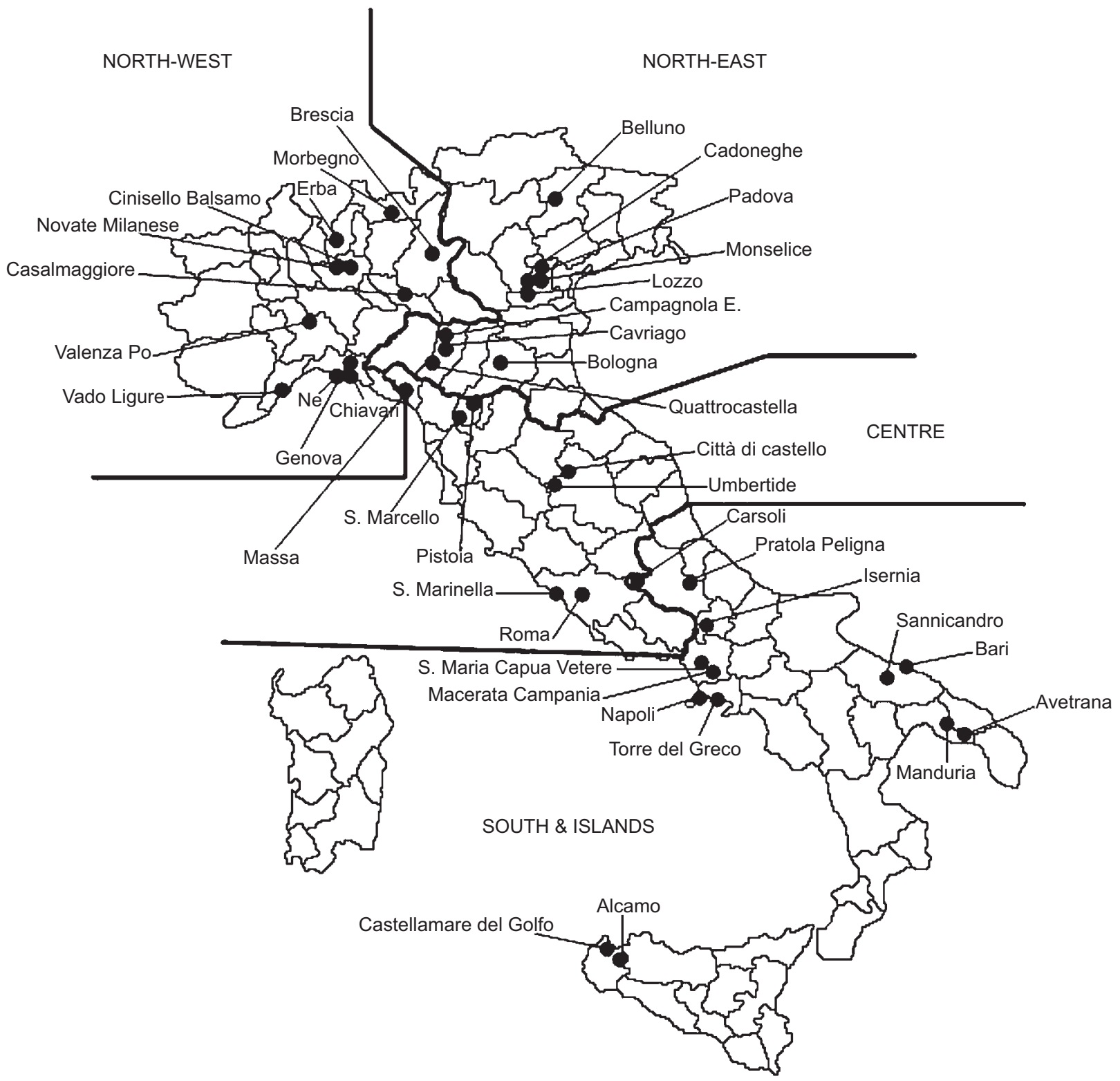

Fig. 1 Location of the forty municipalities and the twenty-three provinces randomly selected to represent the four main geographical areas of Italy: Italian National Food Consumption Survey INRAN-SCAI 2005-06

performed in 2001 by the Italian National Institute of Statistics (ISTAT) ${ }^{(5)}$ was used for the multistage stratification of the sample into: (i) four geographical area strata; (ii) three provinces population size strata (low, medium and large); (iii) two municipalities population size strata (large-medium and small); and (iv) four household composition strata (one member less than 65 years of age, one member aged 65 years and above, two or three members, four or more members). A total of forty municipalities belonging to twenty-three provinces were involved in the survey (Fig. 1).

In each municipality, households were randomly selected from the telephone guide TELECOM (2005 edition) and were phoned up several times during the daytime and in the evening until contact was established. Each contacted household was invited to participate until the municipality's target number of households was reached for the category in terms of household composition. Each individual who had his/her main meals in the household on a regular basis during the period of the survey was considered as a member of the household, even if he/she was not a relative of other members. Criteria for inclusion of the household were that all members would participate in the case of households with up to three members and that no more than one member would refuse to participate in the case of households with more members. Information on the motivation for refusal was collected. The sampled households were distributed in 
the four seasons (excluding Christmas and Easter periods). The survey calendar was organized to capture an adequate proportion of weekdays and weekend days at group level.

\section{Food survey}

The food survey was conducted from October 2005 to December 2006 by a team of thirty trained field workers. Food consumption was self-recorded by subjects for three consecutive days on hard-copy diaries structured by meal. All foods, beverages, food supplements and medicines ingested were to be registered. The survey protocol is described in detail in publications related to previous food surveys performed by the National Research Institute for Food and Nutrition (INRAN) with the same methodology ${ }^{(6,7)}$. For children below 8 years of age and for any subject who was not able to do so, the diaries were filled in by the person who took care of him/her. Each field worker individually met each participant three times during the survey period. For every eating occasion, subjects were asked to carefully record: time, place of consumption, detailed description of foods (or beverages), quantity consumed and brand (for manufactured foods). Portion sizes were reported by subjects with the help of a picture booklet. The booklet was based on a selection of photo series from the original EPIC-SOFT picture book $^{(8)}$, with foods and dishes of different standard portion sizes (small, average or large) relevant for the Italian diet. The booklet included photos of household measures (glasses, spoons, cans, etc.) and instructions to quantify the portions used by children.

For each of the three days, subjects were asked if they were following a particular diet and if the consumption they had reported differed from their usual consumption. Field workers subsequently registered their judgement on the reliability of the information recorded in each single day.

Height and body weight were self-reported.

\section{Ancillary databases}

Four databases were used to transform the data reported by subjects into the weight of single raw ingredients and into the amounts of nutrients consumed: (i) the 'Food descriptors database': (ii) the 'Household unit of measurements database'; (iii) the 'Standard recipes database'; and (iv) the 'Food composition database'. In the 'Household unit of measurement database', the portions estimated by subjects with the help of the picture booklet are linked to the specific weight of each food item. This database contains a total of 9450 entries (weight of standard portions of specific dishes or units of measurement) for 2460 foods, i.e. on average approximately four entries per food.

\section{Data coding, data entry and data processing}

The data management system INRAN-DIARIO3.1 developed by INRAN ${ }^{(9)}$ and used in previous surveys ${ }^{(6,7)}$ was used for data coding, data entry and data processing. The food description reported on the diary was entered as such by the field worker in an open format text field. The quantity consumed was entered together with the unit of measurement (e.g. grams, glasses, spoons) and a food descriptor was selected from the relevant database.

The data entry procedure included a consistency check between units of measurements and food descriptors. A central procedure (MASTER) applied quality control routines.

The predicted energy expenditure (EE) was calculated for each subject based on the reported body weight and the equations reported by the Scientific Committee for Food $^{(10)}$. For infants and children below 10 years of age, equations are available to assess directly predicted EE. For subjects above 10 years of age, the estimated BMR $\left(\mathrm{BMR}_{\mathrm{est}}\right)$ is first calculated and then multiplied by the estimated physical activity level (PAL). In adults and the elderly, the values considered were those of sedentary subjects with 'desirable physical activities'.

Thus, the hard copies of the diaries were checked to identify possible data entry errors when no eating occasion had been entered for one of the meals or when the total energy from food and beverages reported in a diary was more than $120 \%$ or less than $70 \%$ of the predicted EE of the subject.

Once the digit errors and codification errors were corrected, the average food consumption and the average energy intake (EI) during the survey period were calculated for each individual.

During the data processing, mixed dishes were disaggregated into their ingredients. Exceptions were some industrial processed products which contain a major ingredient from one category. Thus, fish fingers are reported in the category 'Fish, seafood and their products' even though they contain breading.

The classification of food items at ingredient level into fifteen large food categories and fifty-one subcategories was based largely on the classification developed by the European Food Safety Authority ${ }^{(11)}$. Any subject who consumed at least one item within the food category on at least one eating occasion during the survey was classified as a consumer of the category.

The water used in the reconstitution of dehydrated products was included in the subcategories 'Tap water' or 'Bottled water' together with water consumed as such. The Appendix provides a list of the major food items classified in each subcategory together with a list of the most frequent minor ingredients of composite foods belonging to other categories.

Subjects were classified according to age category: infants ( $0-2.9$ years), children (3-9.9 years), teenagers (10-17.9 years), adults (18-64.9 years) and elderly (65 years and above).

Statistical analyses were carried out using the SAS for Windows statistical software package release $8 \cdot 01$ (SAS Institute Inc., Cary, NC, USA). 


\section{Results}

Among all households that had been randomly extracted, $14 \%$ could not be contacted (they had moved or were absent) whereas $2 \%$ were contacted but not invited to participate because the target number of households for their category had been reached in the municipality. Among households invited to participate, the participation rate was $33 \%$. The most frequently reported motivation for refusal was lack of time (49\%).

A total of 3328 individuals belonging to 1329 households participated in the food survey. Among these, five subjects were excluded because their food diaries were considered unreliable by the field worker due to a low level of collaboration and repeated omissions in the recording of eating occasions. The final study sample therefore comprised 3323 subjects. Males were aged $0 \cdot 1$ to $92 \cdot 9$ years and females were aged $0 \cdot 1$ to $97 \cdot 7$ years. Physical characteristics of the study sample by age and sex are described in Table 1.

Overall, the analysed records represent 9969 days. Weekdays (Monday to Friday) represented $78 \%$ of all survey days, i.e. slightly more than $5 / 7$. Survey days were proportionally distributed among seasons: $25 \%$ in autumn, $25 \%$ in winter, $26 \%$ in spring and $24 \%$ in summer.

Overall 199 subjects (141 females and fifty-eight males) declared to be on a specific diet during the survey, most often a slimming diet (fifty-nine females and seventeen males). Other diets were related to health conditions or other reasons (e.g. vegetarian diet). Moreover, 208 subjects declared that their food consumption on at least one of the survey days had differed from usual, leading to either increased (e.g. feast) or reduced (e.g. subjects feeling unwell) food consumption.

Mean EI in the study sample (3323 subjects) is reported in Table 2 by age and sex. In the same table, EI and the ratio of EI to predicted $\mathrm{EE}$ are reported in a selected sample of 2890 subjects, after exclusion of females who were either pregnant ( $n$ 19) or lactating ( $n$ 10) and of all subjects who had reported a food consumption pattern different from usual or who were on any kind of diet.

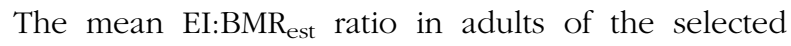
sample was 1.41 (1.36 in males and 1.46 in females; data not in table).

Results in terms of food consumption are presented in Table 3 for the total sample and in Tables $4-11$ by age and sex categories.

The percentage contribution in weight to the total amount of foods and beverages was calculated for each large food category by age and sex and the significance of differences was tested $(P<0 \cdot 05$; Kruskal-Wallis test $)$. Data are illustrated in Fig. 2. For most food categories, the percentage contribution varied significantly among age classes. Statistically significant differences between males and females were observed only in adults and the elderly and only for some food categories. For example, the contribution in weight of 'Alcoholic beverages and substitutes' was higher in males than in females $(7 \cdot 4 \% \mathrm{v}$. $2 \cdot 9 \%$ in adults and $8 \cdot 2 \% v \cdot 3 \cdot 0 \%$ in the elderly).

Among 'Cereals, cereal products and substitutes', the highest consumption was from 'Bread' followed by 'Pasta and pasta substitutes'. The consumption reported for 'Pizza' referred only to plain white pizza and plain tomato pizza. The total pizza consumption (including all types of

Table 1 Physical characteristics of the study sample by age and sex: Italian National Food Consumption Survey INRAN-SCAI 2005-06

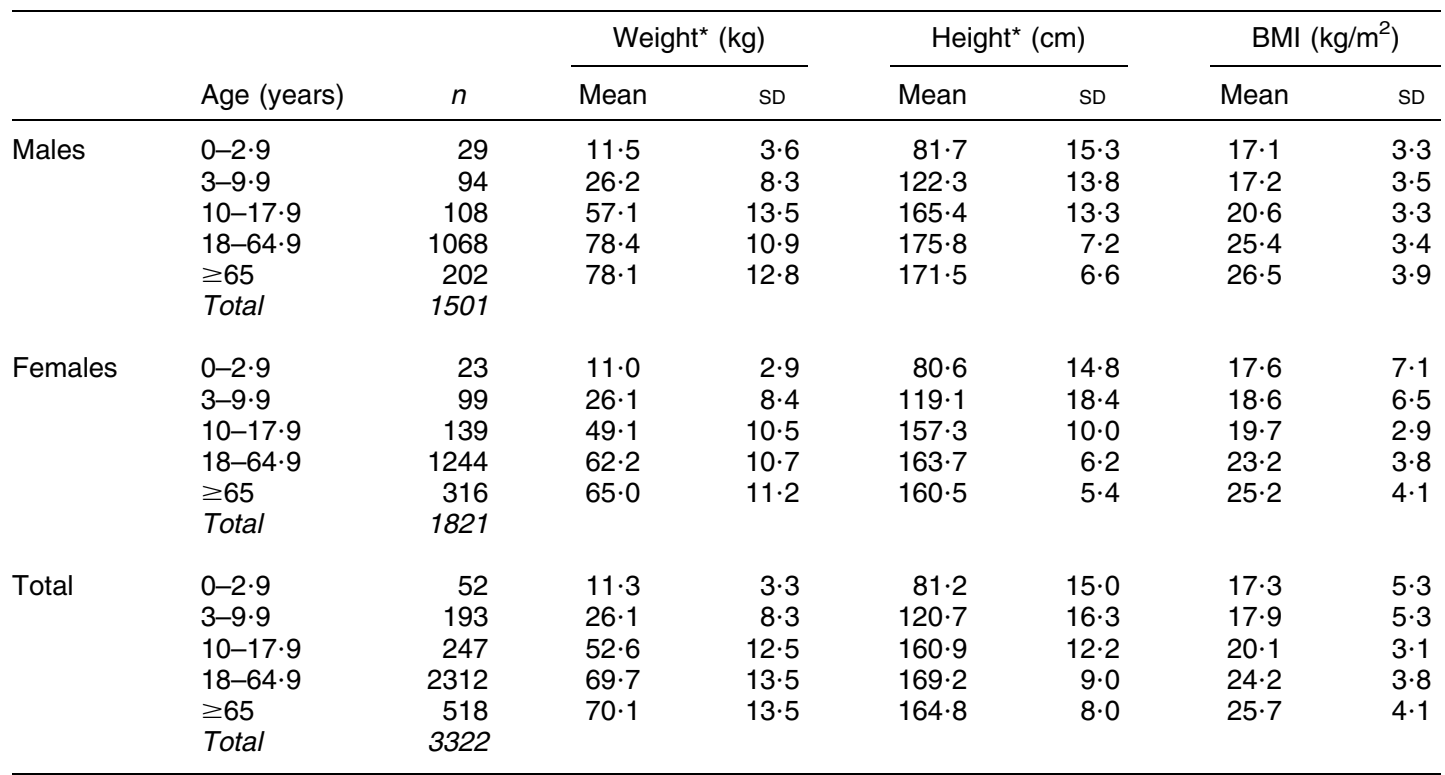

*Weight and height were self-reported. Data are missing for one female in the age class 18-64.9 years. 
Table 2 Estimated energy intake (EI) and ratio of El to predicted energy expenditure (EE) by age and sex: Italian National Food Consumption Survey INRAN-SCAI 2005-06

\begin{tabular}{|c|c|c|c|c|c|c|c|c|c|}
\hline & \multirow[b]{3}{*}{ Age (years) } & \multicolumn{3}{|c|}{ Total study sample } & \multicolumn{5}{|c|}{ Selected sample* } \\
\hline & & \multirow[b]{2}{*}{$n$} & \multicolumn{2}{|c|}{$\mathrm{EI}(\mathrm{MJ})$} & \multirow[b]{2}{*}{$n$} & \multicolumn{2}{|c|}{ El (MJ) } & \multicolumn{2}{|c|}{ El:EE } \\
\hline & & & Mean & SD & & Mean & SD & Mean & SD \\
\hline \multirow[t]{6}{*}{ Males } & $0-2 \cdot 9$ & 29 & $4 \cdot 69$ & $1 \cdot 70$ & 28 & $4 \cdot 65$ & $1 \cdot 72$ & $1 \cdot 04$ & 0.38 \\
\hline & $3-9 \cdot 9$ & 94 & $8 \cdot 17$ & $2 \cdot 15$ & 86 & $8 \cdot 16$ & $2 \cdot 12$ & 0.98 & 0.29 \\
\hline & $10-17 \cdot 9$ & 108 & $10 \cdot 78$ & $3 \cdot 11$ & 101 & $10 \cdot 68$ & $2 \cdot 63$ & $1 \cdot 01$ & 0.27 \\
\hline & $18-64 \cdot 9$ & 1068 & $10 \cdot 01$ & $2 \cdot 72$ & 953 & $10 \cdot 03$ & $2 \cdot 62$ & $0 \cdot 88$ & 0.24 \\
\hline & $\geq 65$ & 202 & $9 \cdot 61$ & $2 \cdot 33$ & 182 & $9 \cdot 70$ & $2 \cdot 28$ & 0.98 & 0.24 \\
\hline & Total & 1501 & & & 1350 & & & & \\
\hline \multirow[t]{6}{*}{ Females } & $0-2 \cdot 9$ & 23 & $4 \cdot 63$ & $1 \cdot 85$ & 21 & $4 \cdot 45$ & $1 \cdot 64$ & 1.03 & 0.37 \\
\hline & $3-9 \cdot 9$ & 99 & $7 \cdot 85$ & 1.93 & 94 & $7 \cdot 77$ & $1 \cdot 91$ & $1 \cdot 03$ & 0.35 \\
\hline & $10-17 \cdot 9$ & 139 & $8 \cdot 75$ & $2 \cdot 22$ & 120 & $8 \cdot 74$ & $2 \cdot 15$ & $1 \cdot 00$ & 0.26 \\
\hline & $18-64 \cdot 9$ & 1245 & $8 \cdot 12$ & $2 \cdot 20$ & 1027 & $8 \cdot 23$ & $2 \cdot 11$ & 0.94 & 0.25 \\
\hline & $\geq 65$ & 316 & $7 \cdot 68$ & 2.03 & 278 & $7 \cdot 80$ & 1.99 & 0.95 & 0.24 \\
\hline & Total & 1822 & & & 1540 & & & & \\
\hline \multirow[t]{6}{*}{ Total } & $0-2 \cdot 9$ & 52 & $4 \cdot 66$ & $1 \cdot 75$ & 49 & $4 \cdot 56$ & $1 \cdot 67$ & $1 \cdot 04$ & 0.37 \\
\hline & $3-9 \cdot 9$ & 193 & $8 \cdot 01$ & $2 \cdot 04$ & 180 & $7 \cdot 96$ & $2 \cdot 02$ & $1 \cdot 00$ & 0.32 \\
\hline & $10-17 \cdot 9$ & 247 & $9 \cdot 64$ & $2 \cdot 83$ & 221 & $9 \cdot 63$ & $2 \cdot 56$ & $1 \cdot 01$ & 0.27 \\
\hline & $18-64 \cdot 9$ & 2313 & 8.99 & $2 \cdot 63$ & 1980 & 9.09 & $2 \cdot 54$ & 0.91 & 0.25 \\
\hline & $\geq 65$ & 518 & $8 \cdot 43$ & $2 \cdot 35$ & 460 & 8.56 & $2 \cdot 30$ & 0.96 & 0.24 \\
\hline & Total & 3323 & & & 2890 & & & & \\
\hline
\end{tabular}

*The selected sample was obtained after exclusion of all female subjects who were either pregnant or lactating and of subjects who had declared to be on any kind of diet or who had reported that their food consumption pattern was different from usual during the survey days.

pizzas with their ingredients) was $55.7 \mathrm{~g} / \mathrm{d}$ in the total population (data not in table).

Among 'Meat, meat products and substitutes', the highest proportion of consumers was found for the subcategory 'Ham, salami, sausages and other preserved meats, excl. offal' ( $81 \%$ of the total sample) but the highest daily consumption was reported for the subcategory 'Beef and veal, not preserved, excl. offal' (more than $40 \mathrm{~g} / \mathrm{d}$ in the total population). Only three subjects declared to follow a vegetarian diet during the survey. Overall thirty-two subjects did not consume 'Meat, meat products and substitutes' during the survey and four subjects consumed only meat substitutes (e.g. seitan and soya hamburger) among this category.

Among 'Oils and fats', olive oil represented $81 \%$ of total consumption (and 93\% of vegetable oils) in the whole sample.

Among 'Alcoholic beverages and substitutes', 'Regular wine and substitutes' represented $70 \%$ of total consumption in the whole sample, reaching $92 \%$ and $94 \%$ respectively in elderly males and elderly females.

A high percentage of infants, children and teenagers appeared to be consumers of 'Alcoholic beverages and substitutes' due to the consumption of dishes which are traditionally prepared with a few drops of alcoholic beverage added during the cooking process. After exclusion of this source, alcoholic beverages were found to be consumed by no infant, by four children $(24 \mathrm{~g} / \mathrm{d}$ on average in consumers) and by fifteen teenagers $(44 \mathrm{~g} / \mathrm{d})$. The overall percentage of consumers of alcoholic beverages - consumed as such - in the study sample was
$46 \cdot 8 \%$ and their mean daily consumption was $194 \cdot 3 \mathrm{~g} / \mathrm{d}$ (data not in table).

\section{Discussion}

\section{Sampling and participation rate}

As expected from the sampling procedure, the distribution of households among geographical areas (257 in the North-West, 376 in the North-East, 257 in the Centre and 439 in the South and Islands) and according to their composition category (329 singles - including 54\% aged 65 years or more, 665 households with two or three members and 665 households with four or more members) was in line with the last population Census ${ }^{(5)}$.

Institutionalized subjects, such as the elderly in rest homes, were not covered.

Use of the TELECOM telephone guide as a sampling basis and of telephone calls as the first contact with households necessarily led to the exclusion of certain typologies of households: (i) households without a fixed telephone (11\% of Italian households according to ISTAT $^{(12)}$ ); (ii) households whose telephone number is not reported in the TELECOM guide; and (iii) households whose members are very frequently out of home and who could not be contacted.

A high percentage of households refused to participate, leading to a low participation rate (33\%). In the previous Italian national food consumption survey ${ }^{(4)}$ the participation rate of households was higher (47\%), but onethird of the subjects were then discarded due to clear 
Table 3 Mean, standard deviation, median and high percentiles of individual daily consumption ( $3 \mathrm{~d}$ average) by food category in the total population and in consumers ( $\mathrm{g} / \mathrm{d}$ ) - all ages, males and females: Italian National Food Consumption Survey INRAN-SCAI 2005-06

\begin{tabular}{|c|c|c|c|c|c|c|c|c|c|c|c|c|}
\hline \multirow[b]{2}{*}{ Food categories } & \multicolumn{5}{|c|}{ Total population ( $n$ 3323) } & \multicolumn{7}{|c|}{ Consumers } \\
\hline & Mean & SD & Median & 95 th* $^{*}$ & 99th* & $n$ & $\%$ & Mean & SD & Median & 95th* & 99th* \\
\hline Cereals, cereal products and substitutes & $258 \cdot 4$ & $106 \cdot 0$ & $248 \cdot 8$ & $443 \cdot 5$ & $590 \cdot 1$ & 3315 & $99 \cdot 8$ & $259 \cdot 0$ & $105 \cdot 3$ & $249 \cdot 3$ & $443 \cdot 5$ & $590 \cdot 1$ \\
\hline Bread & $103 \cdot 3$ & $77 \cdot 4$ & $90 \cdot 0$ & $245 \cdot 0$ & $340 \cdot 0$ & 3061 & $92 \cdot 1$ & $112 \cdot 1$ & $74 \cdot 2$ & $100 \cdot 0$ & $250 \cdot 0$ & $340 \cdot 2$ \\
\hline Pasta and pasta substitutes & $54 \cdot 2$ & $33 \cdot 1$ & $53 \cdot 0$ & $108 \cdot 7$ & $140 \cdot 1$ & 3027 & $91 \cdot 1$ & $59 \cdot 5$ & $29 \cdot 8$ & $56 \cdot 9$ & $110 \cdot 7$ & $141 \cdot 9$ \\
\hline Pizza & $7 \cdot 8$ & $24 \cdot 5$ & 0.0 & $62 \cdot 5$ & $116 \cdot 7$ & 463 & $13 \cdot 9$ & $55 \cdot 7$ & $40 \cdot 4$ & $40 \cdot 0$ & $125 \cdot 0$ & $(250 \cdot 0)$ \\
\hline Rice & $15 \cdot 8$ & $24 \cdot 6$ & $0 \cdot 0$ & $62 \cdot 8$ & $104 \cdot 2$ & 1370 & $41 \cdot 2$ & $38 \cdot 2$ & $24 \cdot 7$ & $32 \cdot 5$ & $83 \cdot 3$ & $126 \cdot 1$ \\
\hline Wheat, other cereals and flours & $36 \cdot 5$ & $36 \cdot 8$ & $26 \cdot 8$ & $105 \cdot 8$ & $147 \cdot 1$ & 2796 & $84 \cdot 1$ & $43 \cdot 4$ & $36 \cdot 2$ & $35 \cdot 7$ & $111 \cdot 1$ & $155 \cdot 0$ \\
\hline Breakfast cereals & 1.5 & $5 \cdot 5$ & $0 \cdot 0$ & $10 \cdot 0$ & $30 \cdot 0$ & 334 & $10 \cdot 1$ & $14 \cdot 8$ & $10 \cdot 4$ & $10 \cdot 0$ & $31 \cdot 3$ & $(49 \cdot 0)$ \\
\hline Biscuits & $13 \cdot 8$ & $21 \cdot 8$ & $2 \cdot 7$ & $53 \cdot 3$ & $93 \cdot 9$ & 1680 & $50 \cdot 6$ & $27 \cdot 3$ & $23 \cdot 9$ & $21 \cdot 3$ & $71 \cdot 4$ & $114 \cdot 7$ \\
\hline Savoury fine bakery products & $8 \cdot 1$ & $16 \cdot 1$ & $0 \cdot 0$ & $36 \cdot 7$ & $66 \cdot 7$ & 1263 & $38 \cdot 0$ & $21 \cdot 2$ & $20 \cdot 1$ & $16 \cdot 0$ & $53 \cdot 3$ & $100 \cdot 0$ \\
\hline Cakes and sweet snacks & $17 \cdot 5$ & $28 \cdot 7$ & 0.0 & $73 \cdot 3$ & $129 \cdot 5$ & 1475 & $44 \cdot 4$ & $39 \cdot 5$ & 31.5 & $31 \cdot 3$ & $102 \cdot 0$ & $156 \cdot 3$ \\
\hline Pulses, fresh and processed & $11 \cdot 3$ & $22 \cdot 9$ & 0.0 & $58 \cdot 7$ & $97 \cdot 2$ & 1149 & $34 \cdot 6$ & $32 \cdot 6$ & $28 \cdot 7$ & $24 \cdot 4$ & $80 \cdot 7$ & $126 \cdot 7$ \\
\hline Vegetables, fresh and processed & $211 \cdot 2$ & 112.5 & $194 \cdot 5$ & $409 \cdot 2$ & $561 \cdot 9$ & 3310 & $99 \cdot 6$ & $212 \cdot 0$ & $111 \cdot 9$ & 194.9 & $409 \cdot 6$ & 561.9 \\
\hline Leafy vegetables, fresh & $43 \cdot 1$ & $52 \cdot 6$ & $27 \cdot 0$ & $143 \cdot 0$ & $243 \cdot 3$ & 2792 & $84 \cdot 0$ & $51 \cdot 3$ & $53 \cdot 6$ & $34 \cdot 2$ & $150 \cdot 9$ & $247 \cdot 8$ \\
\hline Tomatoes, fresh & $41 \cdot 9$ & $42 \cdot 6$ & $31 \cdot 1$ & $125 \cdot 9$ & $196 \cdot 4$ & 2779 & $83 \cdot 6$ & $50 \cdot 1$ & $41 \cdot 9$ & $39 \cdot 8$ & $133 \cdot 0$ & $204 \cdot 8$ \\
\hline Other fruiting vegetables, fresh & $30 \cdot 9$ & 50.5 & $6 \cdot 1$ & $134 \cdot 2$ & $210 \cdot 0$ & 2137 & $64 \cdot 3$ & $48 \cdot 1$ & $56 \cdot 0$ & $26 \cdot 5$ & $156 \cdot 0$ & $238 \cdot 4$ \\
\hline Roots and onions, fresh & $19 \cdot 4$ & $25 \cdot 2$ & $10 \cdot 2$ & $71 \cdot \overline{7}$ & $116 \cdot 9$ & 3249 & $97 \cdot 8$ & $19 \cdot 8$ & $25 \cdot 3$ & $10 \cdot 6$ & $72 \cdot 7$ & $117 \cdot 0$ \\
\hline Other vegetables, fresh & $37 \cdot 3$ & $47 \cdot 0$ & $18 \cdot 2$ & $131 \cdot 4$ & $208 \cdot 3$ & 2754 & $82 \cdot 9$ & $45 \cdot 0$ & $48 \cdot 1$ & $28 \cdot 6$ & $142 \cdot 0$ & $219 \cdot 0$ \\
\hline Vegetables, processed & $36 \cdot 7$ & $33 \cdot 4$ & $31 \cdot \overline{5}$ & $96 \cdot 4$ & $135 \cdot 4$ & 2592 & $78 \cdot 0$ & $47 \cdot 1$ & $30 \cdot 7$ & $39 \cdot 4$ & $101 \cdot 4$ & $145 \cdot 5$ \\
\hline Spices and herbs & 1.9 & $2 \cdot 9$ & $1 \cdot 0$ & $6 \cdot 1$ & $12 \cdot 0$ & 2761 & $83 \cdot 1$ & $2 \cdot 3$ & $3 \cdot 0$ & $1 \cdot 4$ & $6 \cdot 5$ & $14 \cdot 5$ \\
\hline Potatoes, tubers and their products & $50 \cdot 9$ & $55 \cdot 2$ & $41 \cdot 7$ & $156 \cdot 1$ & $219 \cdot 9$ & 2300 & $69 \cdot 2$ & $73 \cdot 6$ & $52 \cdot 3$ & $65 \cdot 4$ & $168 \cdot 3$ & $231 \cdot 5$ \\
\hline Fruit, fresh and processed & $208 \cdot 5$ & $156 \cdot 7$ & $186 \cdot 4$ & $499 \cdot 3$ & $706 \cdot 0$ & 3115 & $93 \cdot 7$ & $222 \cdot 4$ & $152 \cdot 0$ & $198 \cdot 4$ & $506 \cdot 0$ & $712 \cdot 1$ \\
\hline Citrus fruit, fresh & $45 \cdot 9$ & $88 \cdot 3$ & $0 \cdot 0$ & $208 \cdot 7$ & $395 \cdot 3$ & 1559 & $46 \cdot 9$ & $97 \cdot 9$ & $107 \cdot 4$ & $60 \cdot 7$ & $303 \cdot 3$ & $485 \cdot 6$ \\
\hline Exotic fruit, fresh & $17 \cdot 0$ & $28 \cdot 9$ & $0 \cdot 0$ & $66 \cdot 7$ & $130 \cdot 8$ & 1293 & 38.9 & $43 \cdot 7$ & $31 \cdot 4$ & $33 \cdot 3$ & $100 \cdot 0$ & $166 \cdot 7$ \\
\hline Other fruit, fresh & $142 \cdot 5$ & $135 \cdot 4$ & $114 \cdot 5$ & $396 \cdot 7$ & $586 \cdot 7$ & 2760 & $83 \cdot 1$ & $171 \cdot 6$ & $130 \cdot 7$ & $145 \cdot 7$ & $416 \cdot 7$ & $646 \cdot 0$ \\
\hline Nuts, seeds, olives and their products, dried fruit & $2 \cdot 6$ & $6 \cdot 5$ & 0.0 & $13 \cdot 3$ & $27 \cdot 3$ & 899 & $27 \cdot 1$ & $9 \cdot 4$ & $9 \cdot 6$ & $7 \cdot 4$ & $25 \cdot 9$ & $51 \cdot 7$ \\
\hline Other processed fruit (in syrup, in purée, etc.) & 0.5 & $6 \cdot 0$ & $0 \cdot 0$ & $0 \cdot 0$ & $1 \cdot 3$ & 55 & $1 \cdot 7$ & $28 \cdot 9$ & $36 \cdot 7$ & $13 \cdot 3$ & $(106 \cdot 7)$ & $(150 \cdot 0)$ \\
\hline Meat, meat products and substitutes & $110 \cdot 1$ & $62 \cdot 9$ & $101 \cdot 8$ & $221 \cdot 4$ & $306 \cdot 0$ & 3290 & $99 \cdot 0$ & $111 \cdot 2$ & $62 \cdot 3$ & $102 \cdot 8$ & $221 \cdot 7$ & $307 \cdot 6$ \\
\hline Beef and veal, not preserved, excl. offal & $42 \cdot 7$ & $41 \cdot 4$ & $35 \cdot 2$ & $118 \cdot 0$ & $184 \cdot 8$ & 2500 & $75 \cdot 2$ & $56 \cdot 8$ & $38 \cdot 4$ & $45 \cdot 8$ & $129 \cdot 2$ & $204 \cdot 5$ \\
\hline Pork, not preserved, excl. offal & $12 \cdot 7$ & $25 \cdot 9$ & $0 . \overline{0}$ & $58 \cdot 6$ & $105 \cdot 5$ & 1042 & $31 \cdot \overline{4}$ & $40 \cdot 6$ & $31 \cdot 9$ & $41 \cdot 3$ & $93 \cdot \overline{8}$ & $153 \cdot 3$ \\
\hline Poultry and game, not preserved, excl. offal & $20 \cdot 8$ & $29 \cdot 7$ & $0 \cdot 0$ & $79 \cdot 8$ & $117 \cdot 9$ & 1409 & $42 \cdot 4$ & $49 \cdot 0$ & $26 \cdot 5$ & $41 \cdot 7$ & $100 \cdot 3$ & $143 \cdot 6$ \\
\hline Other meats, not preserved, excl. offal & $5 \cdot 5$ & $19 \cdot 5$ & 0.0 & $44 \cdot 1$ & $96 \cdot 4$ & 340 & $10 \cdot 2$ & $53 \cdot 4$ & 33.9 & $41 \cdot 8$ & $111 \cdot 0$ & $(134 \cdot 3)$ \\
\hline $\begin{array}{l}\text { Ham, salami, sausages and other preserved meats, } \\
\text { excl. offal }\end{array}$ & $27 \cdot 3$ & $27 \cdot 4$ & $20 \cdot 0$ & $80 \cdot 2$ & $122 \cdot 1$ & 2701 & $81 \cdot 3$ & $33 \cdot 6$ & $26 \cdot 7$ & $26 \cdot 7$ & $84 \cdot 9$ & $127 \cdot 1$ \\
\hline Offal, blood and their products & $1 \cdot 1$ & $7 \cdot 4$ & 0.0 & $0 \cdot 0$ & $37 \cdot 3$ & 108 & $3 \cdot 3$ & $32 \cdot 7$ & $25 \cdot 2$ & $33 \cdot 3$ & $(70 \cdot 6)$ & $(128 \cdot 7)$ \\
\hline Meat substitutes & $0 \cdot 1$ & $1 \cdot 6$ & 0.0 & $0 \cdot 0$ & 0.0 & 7 & 0.2 & $33 \cdot 3$ & $8 \cdot 3$ & $33 \cdot 3$ & $(50 \cdot 0)$ & $(50 \cdot 0)$ \\
\hline Fish, seafood and their products & $44 \cdot 7$ & $51 \cdot 0$ & $32 \cdot 2$ & $147 \cdot 2$ & $214 \cdot 1$ & 2260 & 68.0 & $65 \cdot 8$ & $49 \cdot 4$ & $57 \cdot 2$ & $165 \cdot 1$ & $230 \cdot 8$ \\
\hline Fish and seafood, fresh and frozen & $38 \cdot 8$ & $49 \cdot 8$ & $12 \cdot \overline{8}$ & $140 \cdot \overline{9}$ & $206 \cdot 0$ & 1816 & $54 \cdot 6$ & $71 \cdot 0$ & $47 \cdot 4$ & $57 \cdot \overline{5}$ & $166 \cdot 4$ & 223.9 \\
\hline Fish and seafood, preserved & $5 \cdot 9$ & $12 \cdot 2$ & 0.0 & $30 \cdot 2$ & $58 \cdot 4$ & 1088 & $32 \cdot 7$ & $18 \cdot 1$ & $15 \cdot 3$ & 13.5 & $50 \cdot 0$ & 73.0 \\
\hline Milk, milk products and substitutes & $198 \cdot 0$ & $129 \cdot 3$ & $185 \cdot 2$ & $416 \cdot 8$ & $570 \cdot 4$ & 3298 & $99 \cdot 2$ & $199 \cdot 5$ & $128 \cdot 7$ & $187 \cdot 1$ & $419 \cdot 9$ & $574 \cdot 7$ \\
\hline $\begin{array}{l}\text { Milk, milk-based beverages, human milk, infant formula } \\
\text { and substitutes }\end{array}$ & $119 \cdot 3$ & $114 \cdot 3$ & $112 \cdot 5$ & $288 \cdot 2$ & $436 \cdot 7$ & 2613 & 78.6 & $151 \cdot 8$ & $108 \cdot 1$ & $150 \cdot 0$ & $308 \cdot 3$ & $467 \cdot 3$ \\
\hline Yoghurt and fermented milk & $20 \cdot 6$ & $48 \cdot 1$ & 0.0 & $125 \cdot 0$ & $208 \cdot 3$ & 793 & 23.9 & $86 \cdot 3$ & $63 \cdot 4$ & $83 \cdot 3$ & $200 \cdot 0$ & $(346 \cdot 7)$ \\
\hline Cheese and substitutes & $57 \cdot 0$ & $42 \cdot 3$ & $50 \cdot 8$ & $133 \cdot 3$ & $190 \cdot 8$ & 3215 & $96 \cdot 7$ & $58 \cdot 9$ & $41 \cdot 6$ & $52 \cdot 5$ & $134 \cdot 8$ & $190 \cdot 8$ \\
\hline Milk-based desserts and substitutes & $1 \cdot 1$ & $7 \cdot 2$ & 0.0 & $5 \cdot 1$ & $31 \cdot 3$ & 212 & $6 \cdot 4$ & $16 \cdot 5$ & $23 \cdot 5$ & $6 \cdot 7$ & $57 \cdot 3$ & $(125 \cdot 0)$ \\
\hline
\end{tabular}




\begin{tabular}{|c|c|c|c|c|c|c|c|c|c|c|c|c|}
\hline \multirow[b]{2}{*}{ Food categories } & \multicolumn{5}{|c|}{ Total population ( $n$ 3323) } & \multicolumn{7}{|c|}{ Consumers } \\
\hline & Mean & SD & Median & $95 \mathrm{th}^{*}$ & 99th* & $n$ & $\%$ & Mean & SD & Median & 95th* & 99 th $^{*}$ \\
\hline Oils and fats & $40 \cdot 4$ & $16 \cdot 7$ & 38.7 & 68.5 & $91 \cdot 0$ & 3312 & $99 \cdot 7$ & $40 \cdot 5$ & $16 \cdot 5$ & $38 \cdot 8$ & $68 \cdot 6$ & $91 \cdot 0$ \\
\hline Olive oil & $32 \cdot 7$ & $13 \cdot 8$ & $31 \cdot 5$ & $56 \cdot 8$ & $71 \cdot 3$ & 3312 & $99 \cdot 7$ & $32 \cdot 9$ & $13 \cdot 6$ & $31 \cdot 5$ & $56 \cdot 8$ & $71 \cdot 3$ \\
\hline Other vegetable oils & $2 \cdot 6$ & $4 \cdot 2$ & 0.0 & $9 \cdot 8$ & $17 \cdot 0$ & 1388 & $41 \cdot 8$ & $6 \cdot 2$ & 4.5 & $6 \cdot 1$ & $12 \cdot 9$ & $24 \cdot 0$ \\
\hline Butter and creams & $4 \cdot 1$ & $8 \cdot 0$ & 0.0 & $18 \cdot 4$ & $34 \cdot 7$ & 1520 & $45 \cdot 7$ & $9 \cdot 0$ & $9 \cdot 8$ & $6 \cdot 1$ & $25 \cdot 1$ & $42 \cdot 2$ \\
\hline Other fats & 0.9 & $2 \cdot 9$ & 0.0 & $6 \cdot 0$ & $15 \cdot 2$ & 595 & $17 \cdot 9$ & $5 \cdot 3$ & $4 \cdot 8$ & 3.9 & $15 \cdot 2$ & $(21 \cdot 3)$ \\
\hline Eggs & $20 \cdot 9$ & $24 \cdot 5$ & $12 \cdot 9$ & $67 \cdot 0$ & $103 \cdot 4$ & 2469 & $74 \cdot 3$ & $28 \cdot 1$ & $24 \cdot 5$ & $20 \cdot 4$ & $73 \cdot 1$ & $115 \cdot 2$ \\
\hline Alcoholic beverages and substitutes & $91 \cdot 0$ & $148 \cdot 8$ & 0.2 & $373 \cdot 5$ & $640 \cdot 1$ & 2477 & 74.5 & $122 \cdot 1$ & $160 \cdot 9$ & $66 \cdot 7$ & $428 \cdot 0$ & $666 \cdot 8$ \\
\hline Regular wine and substitutes & $64 \cdot 1$ & $111 \cdot 2$ & $0 \cdot 1$ & $293 \cdot 3$ & $480 \cdot 0$ & 2315 & $69 \cdot 7$ & $92 \cdot 0$ & $123 \cdot 2$ & $40 \cdot 0$ & $320 \cdot 1$ & $533 \cdot 3$ \\
\hline Beer, cider and substitutes & $24 \cdot 6$ & $79 \cdot 2$ & 0.0 & $133 \cdot 3$ & $400 \cdot 0$ & 550 & $16 \cdot 6$ & $148 \cdot 7$ & $139 \cdot 6$ & $110 \cdot 0$ & $400 \cdot 0$ & $(600 \cdot 0)$ \\
\hline $\begin{array}{l}\text { Sweet wine, spumante, wine-based appetizers, } \\
\text { spirits and liquors }\end{array}$ & $2 \cdot 3$ & $11 \cdot 4$ & 0.0 & $13 \cdot 3$ & $53 \cdot 3$ & 437 & $13 \cdot 2$ & $17 \cdot 4$ & $27 \cdot 0$ & $6 \cdot 7$ & $66 \cdot 8$ & $(133 \cdot 3)$ \\
\hline Sweet products and substitutes & $33 \cdot 1$ & $33 \cdot 3$ & $24 \cdot 0$ & $94 \cdot 6$ & $161 \cdot 8$ & 3096 & $93 \cdot 2$ & $35 \cdot 5$ & $33 \cdot 3$ & $26 \cdot 5$ & $97 \cdot 7$ & $166 \cdot 9$ \\
\hline Ice cream, ice lolly and substitutes & $10 \cdot 0$ & $26 \cdot 2$ & $0 \cdot 0$ & $66 \cdot 7$ & $133 \cdot 3$ & 674 & $20 \cdot 3$ & $49 \cdot 1$ & $38 \cdot 3$ & $33 \cdot 3$ & $133 \cdot 3$ & $(200 \cdot 0)$ \\
\hline Chocolate and substitutes & $2 \cdot 6$ & $7 \cdot 1$ & 0.0 & $15 \cdot 7$ & $34 \cdot 7$ & 753 & $22 \cdot 7$ & $11 \cdot 4$ & $10 \cdot 9$ & $7 \cdot 5$ & 33.3 & $(48 \cdot 1)$ \\
\hline Candies, jam and other sweet products (incl. sugar-free) & $3 \cdot 2$ & $8 \cdot 8$ & 0.0 & $20 \cdot 0$ & $37 \cdot 3$ & 885 & $26 \cdot 6$ & $11 \cdot 8$ & $13 \cdot 7$ & $8 \cdot 4$ & $33 \cdot 3$ & $52 \cdot 5$ \\
\hline Sugar, fructose, honey and other nutritious sweeteners & $16 \cdot 6$ & $15 \cdot 0$ & $14 \cdot 2$ & $43 \cdot 5$ & $62 \cdot 7$ & 2821 & 84.9 & $19 \cdot 6$ & $14 \cdot 4$ & $16 \cdot 0$ & $45 \cdot 6$ & $64 \cdot 3$ \\
\hline Cocoa and cocoa-based powder & $0 \cdot 7$ & $3 \cdot 7$ & 0.0 & $4 \cdot 7$ & $15 \cdot 8$ & 319 & $9 \cdot 6$ & $7 \cdot 6$ & $9 \cdot 4$ & $5 \cdot 2$ & $23 \cdot 3$ & $(36 \cdot 1)$ \\
\hline Artificial sweeteners & 0.0 & 0.3 & 0.0 & 0.0 & $1 \cdot 7$ & 142 & $4 \cdot 3$ & $1 \cdot 1$ & $1 \cdot 2$ & $1 \cdot 0$ & $(3 \cdot 8)$ & $(4 \cdot 7)$ \\
\hline Meal substitutes & 0.0 & $1 \cdot 1$ & 0.0 & 0.0 & 0.0 & 4 & $0 \cdot 1$ & $30 \cdot 1$ & $15 \cdot 2$ & $28 \cdot 5$ & $(50 \cdot 0)$ & $(50 \cdot 0)$ \\
\hline Water and other non-alcoholic beverages & $836 \cdot 1$ & $428 \cdot 8$ & $772 \cdot 1$ & $1640 \cdot 0$ & $2183 \cdot 3$ & 3320 & $99 \cdot 9$ & $836 \cdot 9$ & $428 \cdot 2$ & $772 \cdot 5$ & $1640 \cdot 2$ & $2183 \cdot 3$ \\
\hline Tap water (as such, in beverages or recipes) & $196 \cdot 4$ & $303 \cdot 2$ & $62 \cdot 5$ & $853 \cdot 3$ & $1373 \cdot 3$ & 1897 & $57 \cdot 1$ & $344 \cdot 1$ & $332 \cdot 1$ & $241 \cdot 7$ & $1022 \cdot 6$ & $1493 \cdot 3$ \\
\hline Bottled water & $452 \cdot 2$ & $409 \cdot 9$ & $413 \cdot 3$ & $1173 \cdot 3$ & $1760 \cdot 0$ & 2541 & $76 \cdot 5$ & $591 \cdot 3$ & $370 \cdot 8$ & $533 \cdot 3$ & $1280 \cdot 0$ & $1853 \cdot 3$ \\
\hline Coffee, tea, herbal tea and substitutes & $122 \cdot 8$ & $115 \cdot 5$ & $100 \cdot 0$ & $340 \cdot 0$ & $516 \cdot 7$ & 2913 & $87 \cdot 7$ & $140 \cdot 1$ & $113 \cdot 1$ & $115 \cdot 0$ & $352 \cdot 8$ & $532 \cdot 1$ \\
\hline Fruit and vegetable juices & $36 \cdot 2$ & $80 \cdot 3$ & 1.5 & $200 \cdot 0$ & $336 \cdot 0$ & 1867 & $56 \cdot 2$ & $64 \cdot 4$ & $98 \cdot 2$ & $8 \cdot 4$ & $250 \cdot 0$ & $416 \cdot 7$ \\
\hline Other soft drinks & $28 \cdot \overline{6}$ & $77 \cdot 3$ & 0.0 & $163 \cdot 3$ & $333 \cdot 3$ & 725 & $21 \cdot \overline{8}$ & $130 \cdot 9$ & $118 \cdot 5$ & $106 \cdot 7$ & $330 \cdot 0$ & $(586 \cdot 7)$ \\
\hline Miscellaneous & $3 \cdot 1$ & 3.8 & $2 \cdot 0$ & $10 \cdot 0$ & $17 \cdot 8$ & 2590 & $77 \cdot 9$ & $4 \cdot 0$ & 3.9 & $2 \cdot 7$ & $10 \cdot 8$ & $20 \cdot 0$ \\
\hline Total amount of foods and beveragest & $2118 \cdot 0$ & $629 \cdot 0$ & $2059 \cdot 9$ & $3263 \cdot 3$ & $3900 \cdot 1$ & 3323 & $100 \cdot 0$ & $2118 \cdot 0$ & $629 \cdot 0$ & $2059 \cdot 9$ & $3263 \cdot 3$ & $3900 \cdot 1$ \\
\hline Total amount of liquid foods & $1048 \cdot 8$ & $438 \cdot 5$ & $979 \cdot 4$ & $1886 \cdot 5$ & $2377 \cdot 5$ & 3323 & $100 \cdot 0$ & $1048 \cdot 8$ & $438 \cdot 5$ & $979 \cdot 4$ & $1886 \cdot 5$ & $2377 \cdot 5$ \\
\hline Total amount of solid foods & $1069 \cdot 2$ & $317 \cdot 2$ & $1050 \cdot 6$ & $1607 \cdot 8$ & $1962 \cdot 2$ & 3319 & $99 \cdot 9$ & $1070 \cdot 5$ & $315 \cdot 2$ & $1050 \cdot 9$ & $1608 \cdot 4$ & $1962 \cdot 2$ \\
\hline
\end{tabular}

*High percentiles of consumption assessed on the basis of a $3 \mathrm{~d}$ survey provide an overestimate of long-term high levels of consumption. Values are enclosed in parentheses when the number of subjects to which they

refer is lower than 160 and 800 for the 95th and 99th percentile, respectively, since these values bear a large uncertainty and provide only a rough indication of high levels of consumption. 
Tean, standard deviation, median and high percentiles of individual daily consumption ( $3 \mathrm{~d}$ average) by food category in the total population and in consumers ( $\mathrm{g} / \mathrm{d})-$ infants $(0 \cdot 1$ to $2 \cdot 9$ years), males and females: Italian National Food Consumption Survey INRAN-SCAI 2005-06

\begin{tabular}{|c|c|c|c|c|c|c|c|c|c|c|c|c|}
\hline \multirow[b]{2}{*}{ Food categories } & \multicolumn{5}{|c|}{ Total population ( $n$ 52) } & \multicolumn{7}{|c|}{ Consumers } \\
\hline & Mean & SD & Median & 95th* & 99th* & $n$ & $\%$ & Mean & SD & Median & 95 th $^{*}$ & 99th* \\
\hline Cereals, cereal products and substitutes & $105 \cdot 8$ & $78 \cdot 3$ & $95 \cdot 2$ & $(240 \cdot 6)$ & $(299 \cdot 7)$ & 44 & $84 \cdot 6$ & $125 \cdot 0$ & $69 \cdot 3$ & $111 \cdot 0$ & $(240 \cdot 6)$ & $(299 \cdot 7)$ \\
\hline Bread & $17 \cdot 9$ & $28 \cdot 4$ & 0.0 & $(86 \cdot 0)$ & $(95 \cdot 0)$ & 21 & $40 \cdot 4$ & $44 \cdot 3$ & $28 \cdot 8$ & $37 \cdot 5$ & $(90 \cdot 0)$ & $(95 \cdot 0)$ \\
\hline Pasta and pasta substitutes & $36 \cdot 1$ & $29 \cdot 1$ & $38 \cdot 0$ & $(86 \cdot 2)$ & $(125 \cdot 2)$ & 41 & $78 \cdot 8$ & $45 \cdot 8$ & $25 \cdot 0$ & $43 \cdot 5$ & $(86 \cdot 2)$ & $(125 \cdot 2)$ \\
\hline Pizza & 3.9 & $15 \cdot 4$ & 0.0 & $(37 \cdot 5)$ & $(75 \cdot 0)$ & 4 & $7 \cdot 7$ & $51 \cdot 0$ & $28 \cdot 9$ & $56 \cdot 3$ & $(75 \cdot 0)$ & $(75 \cdot 0)$ \\
\hline Rice & $12 \cdot 6$ & $28 \cdot 4$ & $0 \cdot 0$ & $(77 \cdot 3)$ & $(144 \cdot 0)$ & 15 & $28 \cdot 8$ & $43 \cdot 6$ & $38 \cdot 7$ & $34 \cdot 2$ & $(144 \cdot 0)$ & $(144 \cdot 0)$ \\
\hline Wheat, other cereals and flours & $10 \cdot 8$ & $18 \cdot 3$ & $0 \cdot 2$ & $(54 \cdot 3)$ & $(70 \cdot 0)$ & 26 & $50 \cdot 0$ & $21 \cdot 6$ & $21 \cdot 0$ & $11 \cdot 5$ & $(58 \cdot 9)$ & $(70 \cdot 0)$ \\
\hline Breakfast cereals & 0.3 & $1 \cdot 4$ & $0 \cdot 0$ & $(1 \cdot 7)$ & $(7 \cdot 5)$ & 3 & $5 \cdot 8$ & $5 \cdot 3$ & $3 \cdot 2$ & $6 \cdot 7$ & $(7 \cdot 5)$ & $(7 \cdot 5)$ \\
\hline Biscuits & $16 \cdot 2$ & $14 \cdot 4$ & $14 \cdot 0$ & $(41 \cdot 3)$ & $(48 \cdot 3)$ & 40 & $76 \cdot 9$ & $21 \cdot 0$ & $12 \cdot 8$ & $18 \cdot 0$ & $(43 \cdot 3)$ & $(48 \cdot 3)$ \\
\hline Savoury fine bakery products & $1 \cdot 4$ & $4 \cdot 8$ & $0 \cdot 0$ & $(7 \cdot 5)$ & $(32 \cdot 0)$ & 8 & $15 \cdot 4$ & $9 \cdot 0$ & $9 \cdot 4$ & $5 \cdot 9$ & $(32 \cdot 0)$ & $(32 \cdot 0)$ \\
\hline Cakes and sweet snacks & $6 \cdot 6$ & $19 \cdot 2$ & $0 \cdot 0$ & $(37 \cdot 3)$ & $(110 \cdot 6)$ & 10 & $19 \cdot 2$ & $34 \cdot 3$ & $32 \cdot 1$ & $26 \cdot 7$ & $(110 \cdot 6)$ & $(110 \cdot 6)$ \\
\hline Pulses, fresh and processed & $5 \cdot 2$ & $9 \cdot 0$ & 0.0 & $(30 \cdot 0)$ & $(30 \cdot 2)$ & 15 & $28 \cdot 8$ & $17 \cdot 9$ & $7 \cdot 1$ & $15 \cdot 5$ & $(30 \cdot 2)$ & $(30 \cdot 2)$ \\
\hline Vegetables, fresh and processed & $60 \cdot 5$ & $66 \cdot 3$ & $42 \cdot 7$ & $(235 \cdot 1)$ & $(285 \cdot 7)$ & 44 & $84 \cdot 6$ & $71 \cdot 5$ & $66 \cdot 3$ & 49.9 & $(235 \cdot 1)$ & $(285 \cdot 7)$ \\
\hline Leafy vegetables, fresh & $5 \cdot 3$ & $15 \cdot 0$ & $0 \cdot 0$ & $(30 \cdot 3)$ & $(86 \cdot 4)$ & 19 & $36 \cdot 5$ & $14 \cdot 6$ & $22 \cdot 2$ & 6.9 & $(86 \cdot 4)$ & $(86 \cdot 4)$ \\
\hline Tomatoes, fresh & $6 \cdot 0$ & $10 \cdot 3$ & $0 \cdot 0$ & $(27 \cdot 7)$ & $(50 \cdot 0)$ & 20 & $38 \cdot 5$ & $15 \cdot 7$ & $11 \cdot 2$ & 11.9 & $(40 \cdot 3)$ & $(50 \cdot 0)$ \\
\hline Other fruiting vegetables, fresh & $7 \cdot 2$ & $19 \cdot 3$ & $0 \cdot 0$ & $(53 \cdot 5)$ & $(109 \cdot 5)$ & 15 & $28 \cdot 8$ & $24 \cdot 8$ & $29 \cdot 8$ & $12 \cdot 9$ & $(109 \cdot 5)$ & $(109 \cdot 5)$ \\
\hline Roots and onions, fresh & $9 \cdot 8$ & $15 \cdot 9$ & $5 \cdot 1$ & $(28 \cdot 5)$ & $(95 \cdot 8)$ & 41 & $78 \cdot 8$ & $12 \cdot 4$ & $17 \cdot 0$ & $6 \cdot 5$ & $(28 \cdot 5)$ & $(95 \cdot 8)$ \\
\hline Other vegetables, fresh & $2 \cdot 9$ & $6 \cdot 6$ & $0 \cdot 4$ & $(20 \cdot 9)$ & $(35 \cdot 3)$ & 29 & $55 \cdot 8$ & $5 \cdot 1$ & $8 \cdot 3$ & $2 \cdot 4$ & $(25 \cdot 0)$ & $(35 \cdot 3)$ \\
\hline Vegetables, processed & $29 \cdot 0$ & $46 \cdot 7$ & $23 \cdot 6$ & $(75 \cdot 0)$ & $(240 \cdot 0)$ & 31 & $59 \cdot 6$ & $48 \cdot 7$ & $52 \cdot 2$ & $32 \cdot 2$ & $(231 \cdot 1)$ & $(240 \cdot 0)$ \\
\hline Spices and herbs & 0.3 & 0.8 & $0 \cdot 0$ & $(2 \cdot 9)$ & $(3 \cdot 3)$ & 19 & $36 \cdot 5$ & 0.9 & $1 \cdot 1$ & 0.4 & $(3 \cdot 3)$ & $(3 \cdot 3)$ \\
\hline Potatoes, tubers and their products & $17 \cdot 3$ & $26 \cdot 7$ & $1 \cdot 6$ & $(84 \cdot 5)$ & $(102 \cdot 6)$ & 27 & $51 \cdot 9$ & $33 \cdot 4$ & 28.9 & $19 \cdot 3$ & $(85 \cdot 3)$ & $(102 \cdot 6)$ \\
\hline Fruit, fresh and processed & $117 \cdot 5$ & $99 \cdot 6$ & $93 \cdot 8$ & (283.3) & (363.3) & 44 & $84 \cdot 6$ & $138 \cdot 8$ & $93 \cdot 4$ & $103 \cdot 3$ & (283.3) & (363.3) \\
\hline Citrus fruit, fresh & $7 \cdot 8$ & $22 \cdot 4$ & $0 \cdot 0$ & $(60 \cdot 7)$ & $(121 \cdot 3)$ & 14 & $26 \cdot 9$ & $28 \cdot 9$ & $36 \cdot 3$ & $11 \cdot 7$ & $(121 \cdot 3)$ & $(121 \cdot 3)$ \\
\hline Exotic fruit, fresh & $19 \cdot 2$ & $29 \cdot 8$ & $0 \cdot 0$ & $(83 \cdot 3)$ & (133.3) & 24 & $46 \cdot 2$ & $41 \cdot 6$ & $31 \cdot 5$ & $33 \cdot 3$ & $(88.9)$ & $(133 \cdot 3)$ \\
\hline Other fruit, fresh & $73 \cdot 6$ & $75 \cdot 5$ & $50 \cdot 0$ & $(213 \cdot 1)$ & $(250 \cdot 6)$ & 36 & $69 \cdot 2$ & $106 \cdot 3$ & $68 \cdot 7$ & $94 \cdot 7$ & $(250 \cdot 0)$ & $(250 \cdot 6)$ \\
\hline Nuts, seeds, olives and their products, dried fruit & 0.5 & $2 \cdot 1$ & $0 \cdot 0$ & $(2 \cdot 7)$ & $(12 \cdot 8)$ & 4 & $7 \cdot 7$ & $6 \cdot 3$ & $5 \cdot 0$ & $5 \cdot 1$ & $(12 \cdot 8)$ & $(12 \cdot 8)$ \\
\hline Other processed fruit (in syrup, in purée, etc.) & $16 \cdot 4$ & $31 \cdot 1$ & $0 \cdot 0$ & $(100 \cdot 0)$ & $(106 \cdot 7)$ & 15 & $28 \cdot 8$ & $56 \cdot 9$ & $32 \cdot 6$ & $53 \cdot 3$ & $(106 \cdot 7)$ & $(106 \cdot 7)$ \\
\hline Meat, meat products and substitutes & $55 \cdot 0$ & $37 \cdot 7$ & $57 \cdot 5$ & $(135 \cdot 9)$ & $(160 \cdot 0)$ & 47 & $90 \cdot 4$ & $60 \cdot 8$ & $34 \cdot 8$ & $62 \cdot 5$ & $(135.9)$ & $(160 \cdot 0)$ \\
\hline Beef and veal, not preserved, excl. offal & $25 \cdot 9$ & $27 \cdot 9$ & $20 \cdot 1$ & $(80 \cdot 0)$ & $(106 \cdot 7)$ & 33 & $63 \cdot 5$ & $40 \cdot 8$ & $24 \cdot 8$ & $37 \cdot 5$ & $(80 \cdot 0)$ & $(106 \cdot 7)$ \\
\hline Pork, not preserved, excl. offal & $1 \cdot 3$ & $3 \cdot 3$ & $0 \cdot 0$ & $(8 \cdot 8)$ & $(16 \cdot 7)$ & 8 & $15 \cdot 4$ & $8 \cdot 4$ & $3 \cdot 5$ & $6 \cdot 6$ & $(16 \cdot 7)$ & $(16 \cdot 7)$ \\
\hline Poultry and game, not preserved, excl. offal & $14 \cdot 7$ & $20 \cdot 8$ & $0 \cdot 0$ & $(53 \cdot 3)$ & $(80 \cdot 0)$ & 22 & $42 \cdot 3$ & $34 \cdot 7$ & $18 \cdot 1$ & $27 \cdot 8$ & $(68 \cdot 5)$ & $(80 \cdot 0)$ \\
\hline Other meats, not preserved, excl. offal & $3 \cdot 8$ & $12 \cdot 7$ & $0 \cdot 0$ & $(26 \cdot 7)$ & $(80 \cdot 0)$ & 8 & $15 \cdot 4$ & $25 \cdot 0$ & $23 \cdot 8$ & $19 \cdot 2$ & $(80 \cdot 0)$ & $(80 \cdot 0)$ \\
\hline $\begin{array}{l}\text { Ham, salami, sausages and other preserved meats, } \\
\text { excl. offal }\end{array}$ & $9 \cdot 3$ & $13 \cdot 3$ & $0 \cdot 8$ & $(39 \cdot 5)$ & $(55 \cdot 9)$ & 26 & $50 \cdot 0$ & $18 \cdot 6$ & $13 \cdot 4$ & $13 \cdot 3$ & $(40 \cdot 0)$ & $(55 \cdot 9)$ \\
\hline Offal, blood and their products & \multirow{2}{*}{\multicolumn{2}{|c|}{$\begin{array}{l}\text { Not consumed } \\
\text { Not consumed }\end{array}$}} & & & & 0 & $0 \cdot 0$ & & & & & \\
\hline Meat substitutes & & & & & & 0 & $0 \cdot 0$ & & & & & \\
\hline Fish, seafood and their products & $24 \cdot 0$ & $31 \cdot 4$ & 0.6 & $(85 \cdot 7)$ & $(118 \cdot 1)$ & 26 & $50 \cdot 0$ & $47 \cdot 9$ & $28 \cdot 5$ & $48 \cdot 7$ & $(95 \cdot 6)$ & $(118 \cdot 1)$ \\
\hline Fish and seafood, fresh and frozen & $21 \cdot 6$ & $27 \cdot 8$ & $0 \cdot 0$ & $(85 \cdot 7)$ & $(95 \cdot 6)$ & 24 & $46 \cdot 2$ & $46 \cdot 9$ & $21 \cdot 8$ & $46 \cdot 7$ & $(85 \cdot 7)$ & $(95 \cdot 6)$ \\
\hline Fish and seafood, preserved & $2 \cdot 3$ & $7 \cdot 8$ & 0.0 & $(22 \cdot 5)$ & $(43 \cdot 8)$ & 7 & $13 \cdot 5$ & $17 \cdot 3$ & 14.9 & $13 \cdot 3$ & $(43 \cdot 8)$ & $(43 \cdot 8)$ \\
\hline Milk, milk products and substitutes & $397 \cdot 3$ & $241 \cdot 7$ & $331 \cdot 4$ & (858.3) & $(1080 \cdot 0)$ & 52 & $100 \cdot 0$ & $397 \cdot 3$ & $241 \cdot 7$ & $331 \cdot 4$ & (858.3) & $(1080 \cdot 0)$ \\
\hline $\begin{array}{l}\text { Milk, milk-based beverages, human milk, infant } \\
\text { formula and substitutes }\end{array}$ & $334 \cdot 0$ & $258 \cdot 2$ & $250 \cdot 4$ & $(840 \cdot 0)$ & $(1080 \cdot 0)$ & 51 & $98 \cdot 1$ & $340 \cdot 6$ & $256 \cdot 4$ & $250 \cdot 7$ & $(840 \cdot 0)$ & $(1080 \cdot 0)$ \\
\hline Yoghurt and fermented milk & $37 \cdot 3$ & $49 \cdot 4$ & $10 \cdot 7$ & $(166 \cdot 7)$ & $(175 \cdot 0)$ & 27 & $51 \cdot 9$ & $71 \cdot 9$ & $47 \cdot 1$ & $50 \cdot 0$ & $(166 \cdot 7)$ & $(175 \cdot 0)$ \\
\hline Cheese and substitutes & $24 \cdot 4$ & $22 \cdot 8$ & $20 \cdot 1$ & $(71 \cdot 3)$ & $(95 \cdot 8)$ & 44 & $84 \cdot 6$ & $28 \cdot 9$ & $22 \cdot 0$ & $23 \cdot 3$ & $(71 \cdot 3)$ & $(95 \cdot 8)$ \\
\hline Milk-based desserts and substitutes & 1.5 & $8 \cdot 6$ & 0.0 & $(2 \cdot 6)$ & $(60 \cdot 0)$ & 3 & $5 \cdot 8$ & $26 \cdot 1$ & $30 \cdot 1$ & $15 \cdot 6$ & $(60 \cdot 0)$ & $(60.0)$ \\
\hline
\end{tabular}




\begin{tabular}{|c|c|c|c|c|c|c|c|c|c|c|c|c|}
\hline \multirow[b]{2}{*}{ Food categories } & \multicolumn{5}{|c|}{ Total population ( $n$ 52) } & \multicolumn{7}{|c|}{ Consumers } \\
\hline & Mean & SD & Median & 95th* & 99th* & $n$ & $\%$ & Mean & SD & Median & 95th* & 99th* \\
\hline Oils and fats & $12 \cdot 8$ & $10 \cdot 0$ & $13 \cdot 0$ & $(31 \cdot 9)$ & $(41 \cdot 3)$ & 43 & $82 \cdot 7$ & $15 \cdot 5$ & $8 \cdot 9$ & $14 \cdot 4$ & $(31 \cdot 9)$ & $(41 \cdot 3)$ \\
\hline Olive oil & $11 \cdot 0$ & $8 \cdot 7$ & $10 \cdot 8$ & $(28 \cdot 7)$ & $(37 \cdot 1)$ & 43 & $82 \cdot 7$ & $13 \cdot 4$ & $7 \cdot 8$ & $12 \cdot 0$ & $(28 \cdot 7)$ & $(37 \cdot 1)$ \\
\hline Other vegetable oils & $0 \cdot 6$ & 1.5 & 0.0 & $(4 \cdot 6)$ & $(6 \cdot 1)$ & 8 & $15 \cdot 4$ & 3.6 & $2 \cdot 0$ & $3 \cdot 7$ & $(6 \cdot 1)$ & $(6 \cdot 1)$ \\
\hline Butter and creams & 0.8 & $1 \cdot 9$ & 0.0 & $(5 \cdot 0)$ & $(8 \cdot 6)$ & 12 & $23 \cdot 1$ & 3.5 & $2 \cdot 4$ & $3 \cdot 1$ & $(8 \cdot 6)$ & $(8 \cdot 6)$ \\
\hline Other fats & $0 \cdot 4$ & $1 \cdot 6$ & 0.0 & $(5 \cdot 6)$ & $(8 \cdot 3)$ & 4 & $7 \cdot 7$ & $5 \cdot 5$ & $2 \cdot 7$ & $6 \cdot 0$ & $(8 \cdot 3)$ & $(8 \cdot 3)$ \\
\hline Eggs & $5 \cdot 9$ & $9 \cdot 6$ & 0.0 & $(27 \cdot 6)$ & $(44 \cdot 4)$ & 23 & $44 \cdot 2$ & $13 \cdot 4$ & $10 \cdot 4$ & $11 \cdot 3$ & $(27 \cdot 9)$ & $(44 \cdot 4)$ \\
\hline Alcoholic beverages and substitutes & 0.0 & 0.0 & 0.0 & $(0.0)$ & $(0 \cdot 1)$ & 11 & $21 \cdot 2$ & 0.0 & 0.0 & 0.0 & $(0.1)$ & $(0 \cdot 1)$ \\
\hline Regular wine and substitutes & 0.0 & 0.0 & 0.0 & $(0.0)$ & $(0 \cdot 1)$ & 10 & $19 \cdot \overline{2}$ & $0 \cdot 0$ & $0 \cdot 0$ & 0.0 & $(0 \cdot 1)$ & $(0 \cdot 1)$ \\
\hline Beer, cider and substitutes & \multicolumn{2}{|c|}{ Not consumed } & & & & 0 & $0 \cdot 0$ & & & & & \\
\hline $\begin{array}{l}\text { Sweet wine, spumante, wine-based appetizers, } \\
\text { spirits and liquors }\end{array}$ & 0.0 & 0.0 & 0.0 & $(0 \cdot 0)$ & $(0 \cdot 0)$ & 1 & 1.9 & 0.0 & - & 0.0 & $(0 \cdot 0)$ & $(0.0)$ \\
\hline Sweet products and substitutes & $10 \cdot 0$ & $16 \cdot 3$ & $2 \cdot 7$ & $(41 \cdot 0)$ & $(86 \cdot 7)$ & 27 & $51 \cdot 9$ & $19 \cdot 3$ & $18 \cdot 3$ & $14 \cdot 0$ & $(51 \cdot 2)$ & $(86 \cdot 7)$ \\
\hline Ice cream, ice lolly and substitutes & 1.6 & $6 \cdot 6$ & 0.0 & $(25 \cdot 0)$ & $(33 \cdot 3)$ & 3 & $5 \cdot 8$ & $27 \cdot 8$ & $4 \cdot 8$ & $25 \cdot 0$ & $(33 \cdot 3)$ & (33.3) \\
\hline Chocolate and substitutes & $1 \cdot 8$ & $5 \cdot 6$ & 0.0 & $(12 \cdot 5)$ & $(31 \cdot 3)$ & 8 & $15 \cdot 4$ & $11 \cdot 6$ & $9 \cdot 9$ & $6 \cdot 7$ & $(31 \cdot 3)$ & $(31 \cdot 3)$ \\
\hline Candies, jam and other sweet products (incl. sugar-free) & 0.4 & $1 \cdot 7$ & 0.0 & $(3 \cdot 7)$ & $(10 \cdot 8)$ & 4 & $7 \cdot 7$ & 4.9 & $4 \cdot 4$ & $4 \cdot 3$ & $(10 \cdot 8)$ & $(10 \cdot 8)$ \\
\hline Sugar, fructose, honey and other nutritious sweeteners & $6 \cdot 0$ & $9 \cdot 0$ & 0.8 & $(26 \cdot 7)$ & $(39 \cdot 7)$ & 26 & $50 \cdot 0$ & $12 \cdot 0$ & $9 \cdot 6$ & $8 \cdot 4$ & $(32 \cdot 0)$ & $(39 \cdot 7)$ \\
\hline Cocoa and cocoa-based powder & 0.3 & $1 \cdot 4$ & 0.0 & $(0.0)$ & $(8 \cdot 3)$ & 2 & $3 \cdot 8$ & $7 \cdot 1$ & $1 \cdot 8$ & $7 \cdot 1$ & $(8 \cdot 3)$ & $(8 \cdot 3)$ \\
\hline Artificial sweeteners & \multicolumn{2}{|c|}{ Not consumed } & & & & 0 & $0 \cdot 0$ & & & & & \\
\hline Meal substitutes & \multicolumn{2}{|c|}{ Not consumed } & & & & 0 & $0 \cdot 0$ & & & & & \\
\hline Water and other non-alcoholic beverages & $446 \cdot 8$ & $258 \cdot 6$ & $405 \cdot 8$ & $(886 \cdot 7)$ & $(1006 \cdot 7)$ & 49 & $94 \cdot 2$ & $474 \cdot 2$ & $240 \cdot 4$ & $443 \cdot 7$ & $(886 \cdot 7)$ & $(1006 \cdot 7)$ \\
\hline Tap water (as such, in beverages or recipes) & $101 \cdot 5$ & $139 \cdot 7$ & $40 \cdot 0$ & $(427 \cdot 5)$ & $(618 \cdot 3)$ & 29 & $55 \cdot 8$ & $182 \cdot 0$ & $142 \cdot 6$ & $142 \cdot 5$ & $(493 \cdot 3)$ & $(618 \cdot 3)$ \\
\hline Bottled water & $265 \cdot 3$ & $215 \cdot 9$ & $240 \cdot 0$ & $(746 \cdot 7)$ & $(853 \cdot 3)$ & 42 & $80 \cdot 8$ & 328.5 & $191 \cdot 7$ & $285 \cdot 0$ & $(746 \cdot 7)$ & $(853 \cdot 3)$ \\
\hline Coffee, tea, herbal tea and substitutes & $11 \cdot 6$ & $41 \cdot 6$ & 0.0 & $(100 \cdot 0)$ & $(250 \cdot 0)$ & 9 & $17 \cdot 3$ & $67 \cdot 1$ & $82 \cdot 7$ & $24 \cdot 5$ & $(250 \cdot 0)$ & $(250 \cdot 0)$ \\
\hline Fruit and vegetable juices & $66 \cdot 3$ & $102 \cdot 8$ & 0.0 & $(300 \cdot 0)$ & $(333 \cdot 3)$ & 24 & $46 \cdot 2$ & $143 \cdot 7$ & $108 \cdot 7$ & $137 \cdot 5$ & $(331 \cdot 7)$ & $(333 \cdot 3)$ \\
\hline Other soft drinks & $2 \cdot 1$ & $10 \cdot 4$ & 0.0 & $(0.0)$ & $(53 \cdot 3)$ & 2 & $3 \cdot 8$ & $53 \cdot 3$ & 0.0 & $53 \cdot 3$ & $(53 \cdot 3)$ & $(53 \cdot 3)$ \\
\hline Miscellaneous & 0.9 & 1.5 & 0.3 & $(3 \cdot 0)$ & $(8 \cdot 5)$ & 33 & 63.5 & $1 \cdot 4$ & $1 \cdot 7$ & 0.9 & $(5 \cdot 3)$ & $(8.5)$ \\
\hline Total amount of foods and beveragest & $1259 \cdot 5$ & 366.9 & $1223 \cdot 3$ & $(1880 \cdot 2)$ & $(1983 \cdot 6)$ & 52 & $100 \cdot 0$ & $1259 \cdot 5$ & $366 \cdot 9$ & $1223 \cdot 3$ & $(1880 \cdot 2)$ & $(1983 \cdot 6)$ \\
\hline Total amount of liquid foods & $776 \cdot 2$ & $311 \cdot 0$ & $763 \cdot 8$ & $(1306 \cdot 0)$ & $(1693 \cdot 3)$ & 52 & $100 \cdot 0$ & $776 \cdot 2$ & $311 \cdot 0$ & $763 \cdot 8$ & $(1306 \cdot 0)$ & (1693.3) \\
\hline Total amount of solid foods & $483 \cdot 3$ & $248 \cdot 5$ & $484 \cdot 5$ & $(846 \cdot 8)$ & $(1011 \cdot 3)$ & 48 & $92 \cdot 3$ & $523 \cdot 6$ & $213 \cdot 2$ & $516 \cdot 5$ & $(846 \cdot 8)$ & $(1011 \cdot 3)$ \\
\hline
\end{tabular}

${ }^{*}$ High percentiles of consumption assessed on the basis of a $3 \mathrm{~d}$ survey provide an overestimate of long-term high levels of consumption. Values are enclosed in parentheses when the number of subjects to which they

refer is lower than 160 and 800 for the 95th and 99th percentile, respectively, since these values bear a large uncertainty and provide only a rough indication of high levels of consumption. 
Table 5 Mean, standard deviation, median and high percentiles of individual daily consumption ( $3 \mathrm{~d}$ average) by food category in the total population and in consumers ( $\mathrm{g} / \mathrm{d})$ - children $(3$ to $9 \cdot 9$ years), males and females: Italian National Food Consumption Survey INRAN-SCAI 2005-06

\begin{tabular}{|c|c|c|c|c|c|c|c|c|c|c|c|c|}
\hline \multirow[b]{2}{*}{ Food categories } & \multicolumn{5}{|c|}{ Total population ( $n$ 193) } & \multicolumn{7}{|c|}{ Consumers } \\
\hline & Mean & SD & Median & 95th* & 99th* & $n$ & $\%$ & Mean & SD & Median & 95 th $^{*}$ & 99th* \\
\hline Cereals, cereal products and substitutes & $238 \cdot 0$ & $87 \cdot 6$ & $230 \cdot 6$ & $394 \cdot 4$ & $(448 \cdot 3)$ & 193 & $100 \cdot 0$ & $238 \cdot 0$ & $87 \cdot 6$ & $230 \cdot 6$ & $394 \cdot 4$ & $(448 \cdot 3)$ \\
\hline Bread & $73 \cdot 6$ & $60 \cdot 5$ & $60 \cdot 0$ & $180 \cdot 0$ & $(290 \cdot 0)$ & 172 & $89 \cdot 1$ & $82 \cdot 6$ & $58 \cdot 0$ & $66 \cdot 3$ & $180 \cdot 0$ & $(290 \cdot 0)$ \\
\hline Pasta and pasta substitutes & $58 \cdot 2$ & $28 \cdot 7$ & $56 \cdot 1$ & $104 \cdot 9$ & $(161 \cdot 7)$ & 188 & $97 \cdot 4$ & $59 \cdot 8$ & $27 \cdot 4$ & $56 \cdot 8$ & $104 \cdot 9$ & $(161 \cdot 7)$ \\
\hline Pizza & $7 \cdot 2$ & $19 \cdot 0$ & 0.0 & $50 \cdot 0$ & $(100 \cdot 0)$ & 35 & $18 \cdot 1$ & $39 \cdot 9$ & $26 \cdot 5$ & $33 \cdot 3$ & $(100 \cdot 0)$ & $(112 \cdot 5)$ \\
\hline Rice & $16 \cdot 2$ & $23 \cdot 2$ & $0 \cdot 0$ & $62 \cdot 5$ & $(104 \cdot 2)$ & 89 & $46 \cdot 1$ & $35 \cdot 1$ & $22 \cdot 5$ & $29 \cdot 7$ & $(83 \cdot 3)$ & $(108 \cdot 8)$ \\
\hline Wheat, other cereals and flours & $26 \cdot 5$ & $27 \cdot 8$ & $15 \cdot 9$ & $84 \cdot 1$ & $(101 \cdot 3)$ & 169 & $87 \cdot 6$ & $30 \cdot 2$ & $27 \cdot 7$ & $24 \cdot 4$ & $85 \cdot 0$ & $(101 \cdot 3)$ \\
\hline Breakfast cereals & $3 \cdot 8$ & $7 \cdot 8$ & $0 \cdot 0$ & $22 \cdot 5$ & $(30 \cdot 0)$ & 50 & $25 \cdot 9$ & $14 \cdot 8$ & $8 \cdot 4$ & $13 \cdot 0$ & $(30 \cdot 0)$ & $(31 \cdot 3)$ \\
\hline Biscuits & $18 \cdot 5$ & $20 \cdot 5$ & $13 \cdot 3$ & $61 \cdot 3$ & $(89 \cdot 3)$ & 131 & $67 \cdot 9$ & $27 \cdot 2$ & $19 \cdot 6$ & $23 \cdot 3$ & $(69 \cdot 3)$ & $(89 \cdot 3)$ \\
\hline Savoury fine bakery products & 5.9 & $11 \cdot 2$ & $0 \cdot 0$ & $30 \cdot 0$ & $(48 \cdot 0)$ & 67 & $34 \cdot 7$ & $17 \cdot 1$ & $13 \cdot 0$ & $12 \cdot 9$ & $(38 \cdot 0)$ & $(71 \cdot 6)$ \\
\hline Cakes and sweet snacks & $28 \cdot 1$ & $30 \cdot 4$ & $20 \cdot 0$ & 94.5 & $(119 \cdot 1)$ & 135 & $69 \cdot 9$ & $40 \cdot 2$ & $28 \cdot 9$ & $33 \cdot 0$ & $(101 \cdot 4)$ & $(119 \cdot 1)$ \\
\hline Pulses, fresh and processed & $10 \cdot 1$ & $15 \cdot 5$ & 0.0 & $40 \cdot 0$ & $(62 \cdot 2)$ & 83 & $43 \cdot 0$ & 23.5 & $15 \cdot 7$ & $21 \cdot 9$ & $(44 \cdot 0)$ & $(101 \cdot 2)$ \\
\hline Vegetables, fresh and processed & $134 \cdot 3$ & $67 \cdot 2$ & $119 \cdot 8$ & $264 \cdot 9$ & (306.9) & 192 & $99 \cdot 5$ & $135 \cdot 0$ & $66 \cdot 7$ & $120 \cdot 4$ & 264.9 & $(306.9)$ \\
\hline Leafy vegetables, fresh & $16 \cdot 3$ & $21 \cdot 4$ & $6 \cdot 8$ & $60 \cdot 7$ & $(98.5)$ & 130 & $67 \cdot 4$ & $24 \cdot 2$ & $22 \cdot 1$ & $18 \cdot 6$ & $(79 \cdot 7)$ & $(98.5)$ \\
\hline Tomatoes, fresh & $29 \cdot 2$ & $26 \cdot 5$ & $23 \cdot 1$ & $76 \cdot 2$ & $(118 \cdot 3)$ & 160 & $82 \cdot 9$ & $35 \cdot 2$ & $25 \cdot 2$ & $29 \cdot 4$ & $(88 \cdot 3)$ & $(118 \cdot 3)$ \\
\hline Other fruiting vegetables, fresh & $14 \cdot 9$ & $26 \cdot 7$ & $0 \cdot 2$ & $83 \cdot 7$ & $(107 \cdot 5)$ & 104 & $53 \cdot 9$ & $27 \cdot 7$ & $31 \cdot 1$ & $16 \cdot 5$ & (93.9) & $(107 \cdot 5)$ \\
\hline Roots and onions, fresh & $18 \cdot 0$ & $20 \cdot 7$ & $10 \cdot 3$ & $65 \cdot 5$ & $(101 \cdot 5)$ & 192 & $99 \cdot 5$ & $18 \cdot 0$ & $20 \cdot 8$ & $10 \cdot 4$ & $65 \cdot 5$ & $(101 \cdot 5)$ \\
\hline Other vegetables, fresh & $19 \cdot 2$ & $29 \cdot 1$ & $7 \cdot 0$ & $65 \cdot 4$ & $(181 \cdot 5)$ & 150 & $77 \cdot 7$ & $24 \cdot 7$ & $30 \cdot 9$ & $15 \cdot 1$ & $(69 \cdot 7)$ & $(181 \cdot 5)$ \\
\hline Vegetables, processed & $35 \cdot 2$ & $23 \cdot 9$ & $31 \cdot 5$ & $77 \cdot 8$ & $(94 \cdot 6)$ & 171 & $88 \cdot 6$ & $39 \cdot 7$ & $21 \cdot 6$ & $34 \cdot 7$ & $80 \cdot 9$ & $(94 \cdot 6)$ \\
\hline Spices and herbs & 1.5 & $2 \cdot 0$ & $0 \cdot 7$ & $5 \cdot 4$ & $(7 \cdot 6)$ & 159 & $82 \cdot 4$ & $1 \cdot 8$ & $2 \cdot 1$ & $1 \cdot 1$ & $(5 \cdot 7)$ & $(7 \cdot 6)$ \\
\hline Potatoes, tubers and their products & $47 \cdot 2$ & $46 \cdot 6$ & $43 \cdot 3$ & $144 \cdot 2$ & $(173 \cdot 4)$ & 140 & $72 \cdot 5$ & $65 \cdot 0$ & $42 \cdot 9$ & $58 \cdot 6$ & $(145 \cdot 0)$ & $(173 \cdot 4)$ \\
\hline Fruit, fresh and processed & $136 \cdot 0$ & $93 \cdot 8$ & $122 \cdot 0$ & $287 \cdot 7$ & $(337 \cdot 9)$ & 175 & $90 \cdot 7$ & $150 \cdot 0$ & $87 \cdot 1$ & $138 \cdot 2$ & 288.0 & $(337.9)$ \\
\hline Citrus fruit, fresh & $21 \cdot 9$ & $46 \cdot 8$ & 0.0 & $114 \cdot 0$ & $(240 \cdot 1)$ & 62 & $32 \cdot 1$ & $68 \cdot 2$ & $60 \cdot 8$ & $60 \cdot 7$ & $(182 \cdot 0)$ & $(280 \cdot 0)$ \\
\hline Exotic fruit, fresh & $22 \cdot 6$ & $27 \cdot 7$ & $7 \cdot 5$ & $66 \cdot 7$ & $(100 \cdot 0)$ & 102 & $52 \cdot 8$ & $42 \cdot 7$ & $24 \cdot 3$ & $33 \cdot 3$ & $(100 \cdot 0)$ & $(100 \cdot 0)$ \\
\hline Other fruit, fresh & $89 \cdot 3$ & $82 \cdot 9$ & $74 \cdot 0$ & $237 \cdot 6$ & $(304 \cdot 0)$ & 149 & $77 \cdot 2$ & $115 \cdot 7$ & $76 \cdot 4$ & $100 \cdot 0$ & $(241 \cdot 3)$ & $(304 \cdot 0)$ \\
\hline Nuts, seeds, olives and their products, dried fruit & $1 \cdot 2$ & $3 \cdot 7$ & $0 \cdot 0$ & $7 \cdot 4$ & $(17 \cdot 0)$ & 37 & $19 \cdot 2$ & $6 \cdot 4$ & $6 \cdot 2$ & $5 \cdot 3$ & $(17 \cdot 0)$ & $(34 \cdot 3)$ \\
\hline Other processed fruit (in syrup, in purée, etc.) & $0 . \overline{9}$ & $11 \cdot 0$ & $0 \cdot 0$ & 0.0 & $(33 \cdot 3)$ & 2 & $1 \cdot \overline{0}$ & $91 \cdot 7$ & $82 \cdot \overline{5}$ & $91 \cdot 7$ & $(150 \cdot 0)$ & $(150 \cdot 0)$ \\
\hline Meat, meat products and substitutes & $99 \cdot 6$ & $45 \cdot 3$ & $94 \cdot 6$ & $181 \cdot 8$ & $(237 \cdot 5)$ & 191 & $99 \cdot 0$ & $100 \cdot 6$ & $44 \cdot 4$ & $94 \cdot 9$ & $181 \cdot 8$ & $(237 \cdot 5)$ \\
\hline Beef and veal, not preserved, excl. offal & $38 \cdot 0$ & $33 \cdot 1$ & $35 \cdot 3$ & $94 \cdot 4$ & $(170 \cdot 2)$ & 154 & $79 \cdot 8$ & $47 \cdot 6$ & $30 \cdot 2$ & $41 \cdot 3$ & $(97 \cdot 8)$ & $(170 \cdot 2)$ \\
\hline Pork, not preserved, excl. offal & $11 \cdot 2$ & $19 \cdot 5$ & 0.0 & 46.9 & $(83 \cdot 0)$ & 71 & $36 \cdot 8$ & $30 \cdot 6$ & $21 \cdot 1$ & $33 \cdot 3$ & $(65 \cdot 0)$ & $(96 \cdot 9)$ \\
\hline Poultry and game, not preserved, excl. offal & $23 \cdot 3$ & $30 \cdot 2$ & $15 \cdot 7$ & $72 \cdot 8$ & $(143 \cdot 6)$ & 105 & $54 \cdot 4$ & $42 \cdot 9$ & $29 \cdot 0$ & $38 \cdot 4$ & $(88 \cdot 3)$ & $(143 \cdot 6)$ \\
\hline Other meats, not preserved, excl. offal & $3 \cdot 0$ & $13 \cdot 9$ & $0 \cdot 0$ & $28 \cdot 8$ & $(60 \cdot 0)$ & 14 & $7 \cdot 3$ & $41 \cdot 4$ & $33 \cdot 6$ & $32 \cdot 3$ & $(148 \cdot 1)$ & $(148 \cdot 1)$ \\
\hline $\begin{array}{l}\text { Ham, salami, sausages and other preserved meats, } \\
\text { excl. offal }\end{array}$ & $23 \cdot 3$ & $23 \cdot 2$ & $15 \cdot 0$ & $72 \cdot 4$ & $(88 \cdot 5)$ & 157 & $81 \cdot 3$ & $28 \cdot 6$ & $22 \cdot 6$ & $20 \cdot 0$ & $(74 \cdot 5)$ & $(88 \cdot 5)$ \\
\hline Offal, blood and their products & 0.7 & $5 \cdot 3$ & $0 \cdot 0$ & 0.0 & $(33 \cdot 3)$ & 4 & $2 \cdot 1$ & $34 \cdot 6$ & $14 \cdot 2$ & $29 \cdot 2$ & $(55 \cdot 1)$ & $(55 \cdot 1)$ \\
\hline Meat substitutes & \multicolumn{2}{|c|}{ Not consumed } & & & & 0 & $0 \cdot 0$ & & & & & \\
\hline Fish, seafood and their products & $40 \cdot 3$ & $44 \cdot 9$ & 37.5 & $132 \cdot 0$ & $(210 \cdot 7)$ & 132 & $68 \cdot 4$ & $58 \cdot 9$ & 43.0 & $50 \cdot 8$ & $(154 \cdot 7)$ & $(210 \cdot 7)$ \\
\hline Fish and seafood, fresh and frozen & $37 \cdot 2$ & $43 \cdot 6$ & $37 \cdot 5$ & $125 \cdot 6$ & $(205 \cdot 1)$ & 114 & $59 \cdot 1$ & $63 \cdot 0$ & $40 \cdot 0$ & $51 \cdot 3$ & $(154 \cdot 7)$ & $(205 \cdot 1)$ \\
\hline Fish and seafood, preserved & $3 \cdot 0$ & $6 \cdot 4$ & 0.0 & $18 \cdot 8$ & $(26 \cdot 5)$ & 52 & $26 \cdot 9$ & $11 \cdot 3$ & $7 \cdot 6$ & $9 \cdot 5$ & $(23.5)$ & $(30 \cdot 0)$ \\
\hline Milk, milk products and substitutes & $259 \cdot 1$ & $122 \cdot 5$ & $255 \cdot 7$ & $487 \cdot 1$ & $(696 \cdot 7)$ & 193 & $100 \cdot 0$ & $259 \cdot 1$ & $122 \cdot 5$ & $255 \cdot 7$ & $487 \cdot 1$ & $(696 \cdot 7)$ \\
\hline $\begin{array}{l}\text { Milk, milk-based beverages, human milk, infant } \\
\text { formula and substitutes }\end{array}$ & $197 \cdot 2$ & $115 \cdot 5$ & $202 \cdot 4$ & $400 \cdot 0$ & $(656 \cdot 7)$ & 185 & $95 \cdot 9$ & $205 \cdot 7$ & $110 \cdot 3$ & $210 \cdot 8$ & $400 \cdot 0$ & $(656 \cdot 7)$ \\
\hline Yoghurt and fermented milk & $16 \cdot 7$ & $38 \cdot 1$ & 0.0 & $100 \cdot 0$ & $(200 \cdot 0)$ & 43 & $22 \cdot 3$ & $74 \cdot 9$ & $46 \cdot 6$ & $66 \cdot 7$ & $(141 \cdot 7)$ & $(250 \cdot 0)$ \\
\hline Cheese and substitutes & $44 \cdot 3$ & $34 \cdot 6$ & $40 \cdot 6$ & $110 \cdot 0$ & $(141.5)$ & 187 & $96 \cdot 9$ & $45 \cdot 7$ & $34 \cdot 2$ & $41 \cdot 3$ & $110 \cdot 0$ & $(141.5)$ \\
\hline Milk-based desserts and substitutes & 0.9 & $5 \cdot 7$ & 0.0 & 0.0 & $(41 \cdot 7)$ & 9 & $4 \cdot 7$ & $20 \cdot 0$ & $18 \cdot 9$ & $15 \cdot 4$ & $(60 \cdot 0)$ & $(60 \cdot 0)$ \\
\hline
\end{tabular}




\begin{tabular}{|c|c|c|c|c|c|c|c|c|c|c|c|c|}
\hline \multirow[b]{2}{*}{ Food categories } & \multicolumn{5}{|c|}{ Total population ( $n$ 193) } & \multicolumn{7}{|c|}{ Consumers } \\
\hline & Mean & SD & Median & 95 th $^{\star}$ & 99th* & $n$ & $\%$ & Mean & SD & Median & 95th* & 99 th $^{*}$ \\
\hline Oils and fats & $33 \cdot 2$ & $12 \cdot 8$ & $30 \cdot 9$ & $55 \cdot 0$ & $(80 \cdot 8)$ & 193 & $100 \cdot 0$ & $33 \cdot 2$ & $12 \cdot 8$ & $30 \cdot 9$ & $55 \cdot 0$ & $(80 \cdot 8)$ \\
\hline Olive oil & $26 \cdot 7$ & $10 \cdot 5$ & $25 \cdot 4$ & $46 \cdot 7$ & $(54 \cdot 5)$ & 193 & $100 \cdot 0$ & $26 \cdot 7$ & $10 \cdot 5$ & $25 \cdot 4$ & $46 \cdot 7$ & $(54 \cdot 5)$ \\
\hline Other vegetable oils & $2 \cdot 4$ & $3 \cdot 0$ & $0 \cdot 8$ & $8 \cdot 6$ & $(10 \cdot 4)$ & 97 & $50 \cdot 3$ & $4 \cdot 8$ & $2 \cdot 4$ & $4 \cdot 6$ & $(9 \cdot 8)$ & $(10 \cdot 5)$ \\
\hline Butter and creams & $3 \cdot 4$ & $6 \cdot 5$ & 0.0 & $17 \cdot 1$ & $(29 \cdot 8)$ & 85 & $44 \cdot 0$ & $7 \cdot 8$ & $7 \cdot 8$ & $5 \cdot 0$ & $(23 \cdot 0)$ & $(45 \cdot 0)$ \\
\hline Other fats & $0 \cdot 7$ & $1 \cdot 9$ & 0.0 & $4 \cdot 7$ & $(9 \cdot 1)$ & 37 & $19 \cdot 2$ & $3 \cdot 5$ & $3 \cdot 1$ & $2 \cdot 8$ & $(9 \cdot 1)$ & $(15 \cdot 2)$ \\
\hline Eggs & $20 \cdot 0$ & $24 \cdot 7$ & $11 \cdot 1$ & $65 \cdot 4$ & $(127 \cdot 6)$ & 150 & $77 \cdot 7$ & $25 \cdot 7$ & $25 \cdot 2$ & $18 \cdot 3$ & $(66 \cdot 4)$ & $(127 \cdot 6)$ \\
\hline Alcoholic beverages and substitutes & 0.5 & $5 \cdot 8$ & 0.0 & 0.2 & $(13 \cdot 3)$ & 113 & $58 \cdot 5$ & 0.9 & $7 \cdot 6$ & 0.0 & $(0 \cdot 2)$ & $(13 \cdot 3)$ \\
\hline Regular wine and substitutes & 0.5 & $5 \cdot 8$ & 0.0 & $0 \cdot 1$ & $(13 \cdot 3)$ & 108 & $56 \cdot 0$ & 0.9 & $7 \cdot 8$ & 0.0 & $(0.2)$ & $(13 \cdot 3)$ \\
\hline Beer, cider and substitutes & \multicolumn{2}{|c|}{ Not consumed } & & & & 0 & $0 \cdot 0$ & & & & & \\
\hline $\begin{array}{l}\text { Sweet wine, spumante, wine-based appetizers, } \\
\text { spirits and liquors }\end{array}$ & $0 \cdot 0$ & $0 \cdot 1$ & 0.0 & $0 \cdot 0$ & $(0 \cdot 3)$ & 7 & $3 \cdot 6$ & 0.4 & $0 \cdot 7$ & $0 \cdot 1$ & $(2 \cdot 0)$ & $(2 \cdot 0)$ \\
\hline Sweet products and substitutes & $35 \cdot 8$ & $31 \cdot 9$ & $25 \cdot 3$ & 93.6 & $(140 \cdot 3)$ & 181 & $93 \cdot 8$ & $38 \cdot 1$ & $31 \cdot 6$ & $27 \cdot 3$ & $93 \cdot 6$ & $(140 \cdot 3)$ \\
\hline Ice cream, ice lolly and substitutes & $14 \cdot 1$ & $27 \cdot 4$ & 0.0 & $66 \cdot 7$ & $(116 \cdot 7)$ & 57 & $29 \cdot 5$ & $47 \cdot 7$ & $30 \cdot 7$ & $40 \cdot 0$ & $(110 \cdot 0)$ & $(175 \cdot 0)$ \\
\hline Chocolate and substitutes & $7 \cdot 3$ & $11 \cdot 4$ & 0.0 & $33 \cdot 3$ & $(47 \cdot 2)$ & 95 & $49 \cdot 2$ & $14 \cdot 9$ & $12 \cdot 2$ & $13 \cdot 3$ & $(36 \cdot 7)$ & $(80 \cdot 0)$ \\
\hline Candies, jam and other sweet products (incl. sugar-free) & $2 \cdot 0$ & $4 \cdot 7$ & 0.0 & $13 \cdot 3$ & $(23 \cdot 7)$ & 50 & $25 \cdot 9$ & $7 \cdot 6$ & $6 \cdot 6$ & $5 \cdot 6$ & $(23 \cdot 7)$ & $(26 \cdot 7)$ \\
\hline Sugar, fructose, honey and other nutritious sweeteners & $9 \cdot 7$ & $10 \cdot 0$ & $8 \cdot 0$ & $28 \cdot 9$ & $(53 \cdot 3)$ & 147 & $76 \cdot 2$ & $12 \cdot 8$ & $9 \cdot 6$ & $10 \cdot 7$ & $(31 \cdot 0)$ & $(53 \cdot 3)$ \\
\hline Cocoa and cocoa-based powder & $2 \cdot 6$ & $7 \cdot 7$ & $0 \cdot 0$ & $16 \cdot 3$ & $(30 \cdot 0)$ & 44 & $22 \cdot 8$ & $11 \cdot 6$ & $12 \cdot 7$ & $8 \cdot 3$ & $(28 \cdot 0)$ & $(79 \cdot 3)$ \\
\hline Artificial sweeteners & \multirow{2}{*}{\multicolumn{2}{|c|}{$\begin{array}{l}\text { Not consumed } \\
\text { Not consumed }\end{array}$}} & & & & 0 & $0 \cdot 0$ & & & & & \\
\hline Meal substitutes & & & & & & 0 & $0 \cdot 0$ & & & & & \\
\hline Water and other non-alcoholic beverages & $650 \cdot 0$ & $280 \cdot 8$ & $590 \cdot 0$ & $1238 \cdot 3$ & $(1562 \cdot 5)$ & 193 & $100 \cdot 0$ & $650 \cdot 0$ & $280 \cdot 8$ & $590 \cdot 0$ & $1238 \cdot 3$ & (1562.5) \\
\hline Tap water (as such, in beverages or recipes) & $188 \cdot 1$ & $228 \cdot 6$ & $90 \cdot 0$ & $613 \cdot 3$ & $(920 \cdot 8)$ & 127 & $65 \cdot 8$ & $285 \cdot 9$ & $226 \cdot 9$ & $266 \cdot 7$ & $(746 \cdot 7)$ & $(920 \cdot 8)$ \\
\hline Bottled water & $319 \cdot 5$ & $287 \cdot 3$ & $280 \cdot 0$ & $840 \cdot 0$ & $(1226 \cdot 7)$ & 150 & $77 \cdot 7$ & $411 \cdot 2$ & $261 \cdot 7$ & $373 \cdot 3$ & $(906 \cdot 7)$ & $(1226 \cdot 7)$ \\
\hline Coffee, tea, herbal tea and substitutes & $34 \cdot 4$ & $80 \cdot 9$ & 0.0 & $173 \cdot 3$ & $(406 \cdot 7)$ & 62 & $32 \cdot 1$ & $107 \cdot 0$ & $112 \cdot 8$ & $77 \cdot 5$ & $(350 \cdot 0)$ & $(566 \cdot 7)$ \\
\hline Fruit and vegetable juices & $80 \cdot 0$ & $96 \cdot 0$ & $50 \cdot 0$ & $266 \cdot 7$ & $(366 \cdot 7)$ & 127 & $65 \cdot 8$ & $121 \cdot 6$ & $94 \cdot 6$ & $116 \cdot 7$ & (333.3) & $(366 \cdot 7)$ \\
\hline Other soft drinks & $27 \cdot 9$ & $52 \cdot 5$ & 0.0 & $120 \cdot 0$ & $(273 \cdot 3)$ & 62 & $32 \cdot 1$ & $87 \cdot 0$ & $58 \cdot 9$ & $66 \cdot 7$ & $(200 \cdot 0)$ & $(280 \cdot 0)$ \\
\hline Miscellaneous & $2 \cdot 1$ & $2 \cdot 9$ & $1 \cdot 0$ & $8 \cdot 0$ & $(15 \cdot 5)$ & 141 & $73 \cdot 1$ & $2 \cdot 9$ & $3 \cdot 0$ & $2 \cdot 0$ & $(8 \cdot 8)$ & $(15 \cdot 5)$ \\
\hline Total amount of foods and beveragest & $1706 \cdot 3$ & $416 \cdot 5$ & $1636 \cdot 9$ & $2574 \cdot 9$ & $(2813 \cdot 7)$ & 193 & $100 \cdot 0$ & $1706 \cdot 3$ & $416 \cdot 5$ & $1636 \cdot 9$ & 2574.9 & (2813.7) \\
\hline Total amount of liquid foods & $849 \cdot 2$ & $277 \cdot 8$ & $797 \cdot 4$ & $1427 \cdot 0$ & $(1705 \cdot 7)$ & 193 & $100 \cdot 0$ & $849 \cdot 2$ & $277 \cdot 8$ & $797 \cdot 4$ & $1427 \cdot 0$ & $(1705 \cdot 7)$ \\
\hline Total amount of solid foods & $857 \cdot 1$ & $229 \cdot 2$ & $827 \cdot 1$ & $1232 \cdot 3$ & $(1465 \cdot 0)$ & 193 & $100 \cdot 0$ & $857 \cdot 1$ & $229 \cdot 2$ & $827 \cdot 1$ & $1232 \cdot 3$ & $(1465 \cdot 0)$ \\
\hline
\end{tabular}

*High percentiles of consumption assessed on the basis of a $3 \mathrm{~d}$ survey provide an overestimate of long-term high levels of consumption. Values are enclosed in parentheses when the number of subjects to which they

refer is lower than 160 and 800 for the 95th and 99th percentile, respectively, since these values bear a large uncertainty and provide only a rough indication of high levels of consumption. 
17.9 years), males: Italian National Food Consumption Survey INRAN-SCAI 2005-06

\begin{tabular}{|c|c|c|c|c|c|c|c|c|c|c|c|c|}
\hline \multirow[b]{2}{*}{ Food categories } & \multicolumn{5}{|c|}{ Total population ( $n$ 108) } & \multicolumn{7}{|c|}{ Consumers } \\
\hline & Mean & SD & Median & 95th* & 99th* & $n$ & $\%$ & Mean & SD & Median & 95th* & 99th* \\
\hline Cereals, cereal products and substitutes & $331 \cdot 3$ & $123 \cdot 3$ & $324 \cdot 0$ & $(550 \cdot 1)$ & $(657 \cdot 3)$ & 108 & $100 \cdot 0$ & $331 \cdot 3$ & $123 \cdot 3$ & $324 \cdot 0$ & $(550 \cdot 1)$ & $(657 \cdot 3)$ \\
\hline Bread & $121 \cdot 4$ & $81 \cdot 4$ & $113 \cdot 0$ & $(280 \cdot 0)$ & $(370 \cdot 0)$ & 105 & $97 \cdot 2$ & $124 \cdot 9$ & $79 \cdot 9$ & $119 \cdot 9$ & $(280 \cdot 0)$ & $(370 \cdot 0)$ \\
\hline Pasta and pasta substitutes & $63 \cdot 6$ & $32 \cdot 4$ & $61 \cdot 8$ & $(128 \cdot 0)$ & $(133 \cdot 3)$ & 103 & $95 \cdot \overline{4}$ & $66 \cdot 7$ & $29 \cdot 9$ & $63 \cdot 3$ & $(128 \cdot 0)$ & $(133 \cdot 3)$ \\
\hline Pizza & $10 \cdot 3$ & $23 \cdot 5$ & 0.0 & $(56 \cdot 7)$ & $(100 \cdot 0)$ & 24 & $22 \cdot 2$ & $46 \cdot 2$ & $29 \cdot 1$ & $40 \cdot 0$ & $(100 \cdot 0)$ & $(133 \cdot 3)$ \\
\hline Rice & $16 \cdot 7$ & $23 \cdot 6$ & $0 \cdot 0$ & $(55 \cdot 3)$ & $(76 \cdot 0)$ & 49 & $45 \cdot 4$ & $36 \cdot 7$ & $22 \cdot 1$ & $33 \cdot 9$ & $(70 \cdot 0)$ & $(146 \cdot 6)$ \\
\hline Wheat, other cereals and flours & $50 \cdot 6$ & $49 \cdot 5$ & $45 \cdot 7$ & $(149 \cdot 3)$ & $(195 \cdot 4)$ & 96 & $88 \cdot 9$ & $57 \cdot 0$ & $49 \cdot 0$ & $54 \cdot 3$ & $(151 \cdot 6)$ & $(304 \cdot 8)$ \\
\hline Breakfast cereals & $3 \cdot 9$ & $8 \cdot 8$ & 0.0 & $(27 \cdot 5)$ & $(33 \cdot 3)$ & 24 & $22 \cdot 2$ & $17 \cdot 5$ & $10 \cdot 4$ & $15 \cdot 8$ & $(33 \cdot 3)$ & $(36 \cdot 7)$ \\
\hline Biscuits & $28 \cdot 9$ & $43 \cdot 8$ & $16 \cdot 0$ & $(104 \cdot 7)$ & $(215 \cdot 3)$ & 65 & $60 \cdot 2$ & $48 \cdot 0$ & $47 \cdot 7$ & $37 \cdot 3$ & $(144 \cdot 0)$ & $(270 \cdot 0)$ \\
\hline Savoury fine bakery products & $7 \cdot 4$ & $14 \cdot 0$ & 0.0 & $(40 \cdot 0)$ & $(53 \cdot 7)$ & 41 & $38 \cdot 0$ & $19 \cdot 4$ & $16 \cdot 9$ & $13 \cdot 3$ & $(53 \cdot 3)$ & $(85 \cdot 7)$ \\
\hline Cakes and sweet snacks & $28 \cdot 6$ & $39 \cdot 3$ & $16 \cdot 8$ & $(121 \cdot 8)$ & $(180 \cdot 0)$ & 70 & $64 \cdot 8$ & $44 \cdot 1$ & $41 \cdot 2$ & $33 \cdot 3$ & $(138 \cdot 4)$ & $(218 \cdot 8)$ \\
\hline Pulses, fresh and processed & $13 \cdot 8$ & $26 \cdot 6$ & 0.0 & $(76.5)$ & $(119 \cdot 0)$ & 36 & $33 \cdot 3$ & $41 \cdot 4$ & $31 \cdot 4$ & $30 \cdot 3$ & $(119 \cdot 0)$ & (122.6) \\
\hline Vegetables, fresh and processed & $186 \cdot 3$ & $107 \cdot 9$ & $165 \cdot 6$ & $(390 \cdot 4)$ & $(493 \cdot 6)$ & 108 & $100 \cdot 0$ & $186 \cdot 3$ & $107 \cdot 9$ & $165 \cdot 6$ & $(390 \cdot 4)$ & $(493 \cdot 6)$ \\
\hline Leafy vegetables, fresh & $32 \cdot 9$ & $50 \cdot 4$ & $13 \cdot 5$ & $(131 \cdot 5)$ & $(184 \cdot 3)$ & 81 & $75 \cdot 0$ & $43 \cdot 9$ & $54 \cdot 0$ & $24 \cdot 8$ & $(154.9)$ & (333.3) \\
\hline Tomatoes, fresh & $38 \cdot 6$ & $37 \cdot 5$ & $30 \cdot 7$ & $(114.9)$ & $(172 \cdot 6)$ & 90 & $83 \cdot 3$ & $46 \cdot 3$ & $36 \cdot 5$ & $34 \cdot 8$ & $(118 \cdot 7)$ & $(204 \cdot 3)$ \\
\hline Other fruiting vegetables, fresh & $19 \cdot 1$ & $37 \cdot 9$ & $0 \cdot 2$ & $(113 \cdot 2)$ & $(132 \cdot 2)$ & 59 & $54 \cdot 6$ & $35 \cdot 0$ & $45 \cdot 7$ & $8 \cdot 2$ & $(122 \cdot 6)$ & $(182 \cdot 2)$ \\
\hline Roots and onions, fresh & $16 \cdot 5$ & $18 \cdot 6$ & $9 \cdot 3$ & $(62.9)$ & $(81 \cdot 0)$ & 108 & $100 \cdot 0$ & $16 \cdot 5$ & $18 \cdot 6$ & $9 \cdot 3$ & $(62.9)$ & $(81 \cdot 0)$ \\
\hline Other vegetables, fresh & $28 \cdot 7$ & $34 \cdot 2$ & $14 \cdot 2$ & $(87 \cdot 9)$ & $(108 \cdot 8)$ & 89 & $82 \cdot 4$ & $34 \cdot 9$ & $34 \cdot 7$ & $21 \cdot 3$ & $(97 \cdot 1)$ & $(181 \cdot 5)$ \\
\hline Vegetables, processed & $48 \cdot 5$ & $39 \cdot 4$ & $40 \cdot 2$ & $(120 \cdot 4)$ & $(181 \cdot 1)$ & 90 & $83 \cdot 3$ & $58 \cdot 2$ & $36 \cdot 0$ & $50 \cdot 6$ & $(126 \cdot 0)$ & $(186 \cdot 0)$ \\
\hline Spices and herbs & 1.9 & $2 \cdot 2$ & $1 \cdot 1$ & $(6 \cdot 5)$ & $(8 \cdot 1)$ & 91 & $84 \cdot 3$ & $2 \cdot \overline{3}$ & $2 \cdot 2$ & $1 \cdot 6$ & $(6 \cdot 7)$ & $(9 \cdot 7)$ \\
\hline Potatoes, tubers and their products & $68 \cdot 7$ & $66 \cdot 2$ & $63 \cdot 5$ & $(185 \cdot 7)$ & $(232 \cdot 6)$ & 83 & $76 \cdot 9$ & $89 \cdot 4$ & $62 \cdot 0$ & $81 \cdot 7$ & $(210 \cdot 4)$ & $(327 \cdot 2)$ \\
\hline Fruit, fresh and processed & $139 \cdot 2$ & $114 \cdot 6$ & $118 \cdot 0$ & $(351 \cdot 1)$ & $(435 \cdot 6)$ & 98 & $90 \cdot 7$ & $153 \cdot 4$ & $110 \cdot 9$ & $128 \cdot 9$ & $(352 \cdot 7)$ & $(621 \cdot 0)$ \\
\hline Citrus fruit, fresh & $25 \cdot \overline{8}$ & $55 \cdot 7$ & 0.0 & $(174 \cdot 7)$ & $(242 \cdot 7)$ & 47 & 43.5 & $59 \cdot 2$ & $72 \cdot 0$ & $26 \cdot 7$ & $(222 \cdot 7)$ & $(274 \cdot 0)$ \\
\hline Exotic fruit, fresh & $23 \cdot 2$ & $33 \cdot 9$ & $0 \cdot 0$ & $(100 \cdot 0)$ & $(128 \cdot 3)$ & 46 & $42 \cdot 6$ & $54 \cdot 4$ & $31 \cdot 6$ & $50 \cdot 0$ & $(106 \cdot 3)$ & $(133 \cdot 3)$ \\
\hline Other fruit, fresh & $89 \cdot 2$ & $101 \cdot 6$ & $56 \cdot 0$ & $(273 \cdot 2)$ & $(415 \cdot 1)$ & 80 & $74 \cdot 1$ & $120 \cdot 4$ & $100 \cdot 8$ & $90 \cdot 2$ & $(289 \cdot 2)$ & $(617 \cdot 0)$ \\
\hline Nuts, seeds, olives and their products, dried fruit & $1 \cdot 0$ & $3 \cdot 3$ & 0.0 & $(7 \cdot 4)$ & $(10 \cdot 7)$ & 16 & $14 \cdot 8$ & $7 \cdot 0$ & $5 \cdot 8$ & $6 \cdot 6$ & $(25 \cdot 9)$ & $(25 \cdot 9)$ \\
\hline Other processed fruit (in syrup, in purée, etc.) & $0 \cdot 0$ & $0 \cdot 1$ & $0 \cdot 0$ & $(0 \cdot 0)$ & $(0 \cdot 0)$ & 1 & 0.9 & $1 \cdot 3$ & - & $1 \cdot 3$ & $(1 \cdot 3)$ & $(1 \cdot 3)$ \\
\hline Meat, meat products and substitutes & $145 \cdot 1$ & $69 \cdot 3$ & $129 \cdot 6$ & $(273 \cdot 7)$ & $(393 \cdot 2)$ & 108 & $100 \cdot 0$ & $145 \cdot 1$ & $69 \cdot 3$ & $129 \cdot 6$ & $(273 \cdot 7)$ & (393.2) \\
\hline Beef and veal, not preserved, excl. offal & $60 \cdot 0$ & $48 \cdot 1$ & $50 \cdot 0$ & $(133 \cdot 6)$ & $(187 \cdot 4)$ & 96 & 88.9 & $67 \cdot 5$ & $45 \cdot 8$ & $56 \cdot 3$ & $(136 \cdot 7)$ & $(339 \cdot 3)$ \\
\hline Pork, not preserved, excl. offal & $15 \cdot 7$ & $35 \cdot 4$ & 0.0 & $(79 \cdot 4)$ & $(153 \cdot 3)$ & 35 & $32 \cdot 4$ & $48 \cdot 5$ & $48 \cdot 0$ & $41 \cdot 7$ & $(153 \cdot 3)$ & $(251 \cdot 8)$ \\
\hline Poultry and game, not preserved, excl. offal & $24 \cdot 9$ & $30 \cdot 2$ & $20 \cdot 2$ & $(75 \cdot 0)$ & $(106 \cdot 3)$ & 58 & $53 \cdot 7$ & $46 \cdot 4$ & $26 \cdot 4$ & $41 \cdot 6$ & $(85 \cdot 2)$ & $(188 \cdot 1)$ \\
\hline Other meats, not preserved, excl. offal & $3 \cdot 4$ & $12 \cdot 9$ & $0 \cdot 0$ & $(33 \cdot 3)$ & $(65 \cdot 8)$ & 8 & $7 \cdot 4$ & $46 \cdot 2$ & $17 \cdot 2$ & $41 \cdot 6$ & $(79 \cdot 4)$ & $(79 \cdot 4)$ \\
\hline $\begin{array}{l}\text { Ham, salami, sausages and other preserved meats, } \\
\text { excl. offal }\end{array}$ & $40 \cdot 5$ & 33.9 & $35 \cdot 6$ & $(107 \cdot 9)$ & $(167 \cdot 3)$ & 98 & $90 \cdot 7$ & $44 \cdot \overline{7}$ & $32 \cdot 8$ & $40 \cdot 5$ & $(112 \cdot 4)$ & $(180 \cdot 0)$ \\
\hline Offal, blood and their products & 0.6 & $3 \cdot 8$ & $0 \cdot 0$ & $(0 \cdot 0)$ & $(10 \cdot 7)$ & 4 & $3 \cdot 7$ & $15 \cdot 5$ & $14 \cdot 6$ & $9 \cdot 1$ & $(37 \cdot 3)$ & $(37 \cdot 3)$ \\
\hline Meat substitutes & Not cor & umed & & & & 0 & 0.0 & & & & & \\
\hline Fish, seafood and their products & $48 \cdot 4$ & $55 \cdot 5$ & $37 \cdot 5$ & $(165 \cdot 2)$ & $(197 \cdot 9)$ & 70 & $64 \cdot 8$ & $74 \cdot 6$ & $52 \cdot 9$ & $59 \cdot 7$ & $(173 \cdot 6)$ & $(231 \cdot 4)$ \\
\hline Fish and seafood, fresh and frozen & $43 \cdot 9$ & $55 \cdot 3$ & $11 \cdot 1$ & $(153.9)$ & $(197 \cdot 9)$ & 57 & $52 \cdot 8$ & $83 \cdot 1$ & $50 \cdot 2$ & $69 \cdot 2$ & $(181 \cdot 8)$ & $(230 \cdot 0)$ \\
\hline Fish and seafood, preserved & 4.5 & $10 \cdot 4$ & 0.0 & $(30 \cdot 0)$ & $(43 \cdot 8)$ & 29 & $26 \cdot 9$ & $16 \cdot 7$ & $14 \cdot \overline{2}$ & $12 \cdot \overline{4}$ & $(43 \cdot 8)$ & $(61 \cdot 9)$ \\
\hline Milk, milk products and substitutes & $246 \cdot 0$ & $136 \cdot 6$ & $250 \cdot 5$ & $(493 \cdot 1)$ & $(607 \cdot 2)$ & 106 & $98 \cdot 1$ & $250 \cdot 7$ & $133 \cdot 6$ & $251 \cdot 9$ & (493.1) & $(607 \cdot 2)$ \\
\hline $\begin{array}{l}\text { Milk, milk-based beverages, human milk, infant formula } \\
\text { and substitutes }\end{array}$ & $168 \cdot 3$ & $117 \cdot 1$ & $166 \cdot 7$ & $(333 \cdot 3)$ & $(440 \cdot 0)$ & 94 & $87 \cdot 0$ & $193 \cdot 3$ & $104 \cdot 3$ & $181 \cdot 2$ & $(364 \cdot 8)$ & $(595 \cdot 8)$ \\
\hline Yoghurt and fermented milk & $13 \cdot 5$ & $35 \cdot 2$ & 0.0 & $(83 \cdot 3)$ & $(135 \cdot 3)$ & 21 & $19 \cdot 4$ & $69 \cdot 2$ & $51 \cdot 0$ & $52 \cdot 0$ & $(135 \cdot 3)$ & $(250 \cdot 0)$ \\
\hline Cheese and substitutes & $63 \cdot 8$ & $44 \cdot 1$ & $61 \cdot 4$ & $(136 \cdot 6)$ & $(187 \cdot 6)$ & 104 & $96 \cdot 3$ & $66 \cdot 2$ & $43 \cdot 1$ & $62 \cdot 7$ & $(136 \cdot 6)$ & $(187 \cdot 6)$ \\
\hline Milk-based desserts and substitutes & 0.5 & $3 \cdot 0$ & 0.0 & $(0.0)$ & $(15 \cdot 4)$ & 5 & $4 \cdot 6$ & $10 \cdot \overline{7}$ & $10 \cdot 4$ & $5 \cdot 1$ & $(26 \cdot 7)$ & $(26 \cdot 7)$ \\
\hline
\end{tabular}




\begin{tabular}{|c|c|c|c|c|c|c|c|c|c|c|c|c|}
\hline \multirow[b]{2}{*}{ Food categories } & \multicolumn{5}{|c|}{ Total population ( $n$ 108) } & \multicolumn{7}{|c|}{ Consumers } \\
\hline & Mean & SD & Median & 95th* & 99th* & $n$ & $\%$ & Mean & SD & Median & 95th* & 99th* \\
\hline Oils and fats & $44 \cdot 8$ & $17 \cdot 3$ & $41 \cdot 6$ & $(76 \cdot 2)$ & $(97 \cdot 2)$ & 108 & $100 \cdot 0$ & $44 \cdot 8$ & $17 \cdot 3$ & $41 \cdot 6$ & $(76 \cdot 2)$ & $(97 \cdot 2)$ \\
\hline Olive oil & $35 \cdot 1$ & $12 \cdot 9$ & $32 \cdot 0$ & $(61 \cdot 1)$ & $(69 \cdot 8)$ & 108 & $100 \cdot 0$ & $35 \cdot 1$ & $12 \cdot 9$ & $32 \cdot 0$ & $(61 \cdot 1)$ & $(69 \cdot 8)$ \\
\hline Other vegetable oils & $4 \cdot 2$ & $8 \cdot 8$ & $3 \cdot 0$ & $(10 \cdot 5)$ & $(24 \cdot 0)$ & 61 & $56 \cdot 5$ & $7 \cdot 5$ & $10 \cdot 7$ & $6 \cdot 1$ & $(12 \cdot 8)$ & $(84 \cdot 5)$ \\
\hline Butter and creams & $4 \cdot 2$ & $6 \cdot 2$ & $1 \cdot 3$ & $(16 \cdot 7)$ & $(30 \cdot 2)$ & 57 & $52 \cdot 8$ & $7 \cdot 9$ & $6 \cdot 7$ & $6 \cdot 5$ & $(24 \cdot 4)$ & $(30 \cdot 3)$ \\
\hline Other fats & $1 \cdot 3$ & $3 \cdot 1$ & 0.0 & $(5 \cdot 3)$ & $(15 \cdot 2)$ & 31 & $28 \cdot 7$ & $4 \cdot 6$ & $4 \cdot 2$ & $3 \cdot 3$ & $(15 \cdot 2)$ & $(17 \cdot 3)$ \\
\hline Eggs & $21 \cdot 0$ & $24 \cdot 7$ & $12 \cdot 4$ & $(67 \cdot 2)$ & (119.7) & 89 & $82 \cdot 4$ & $25 \cdot 5$ & $25 \cdot 0$ & $18 \cdot 7$ & $(70 \cdot 1)$ & $(126 \cdot 8)$ \\
\hline Alcoholic beverages and substitutes & $4 \cdot 7$ & $18 \cdot 5$ & 0.0 & $(40 \cdot 0)$ & $(66 \cdot 8)$ & 70 & $64 \cdot 8$ & $7 \cdot 3$ & $22 \cdot 6$ & $0 \cdot 1$ & $(53 \cdot 3)$ & $(150 \cdot 1)$ \\
\hline Regular wine and substitutes & $2 \cdot 6$ & $9 \cdot 7$ & 0.0 & $(26 \cdot 8)$ & $(53 \cdot 3)$ & 66 & $61 \cdot 1$ & $4 \cdot 2$ & $12 \cdot 2$ & $0 \cdot 1$ & $(40 \cdot 0)$ & $(53.4)$ \\
\hline Beer, cider and substitutes & $1 \cdot 6$ & $12 \cdot 3$ & 0.0 & $(0 \cdot 0)$ & $(66 \cdot 7)$ & 2 & $1 \cdot 9$ & $88 \cdot 3$ & $30 \cdot 6$ & $88 \cdot 3$ & $(110 \cdot 0)$ & $(110 \cdot 0)$ \\
\hline $\begin{array}{l}\text { Sweet wine, spumante, wine-based appetizers, } \\
\text { spirits and liquors }\end{array}$ & 0.5 & $5 \cdot 1$ & 0.0 & $(0 \cdot 1)$ & $(0.9)$ & 10 & $9 \cdot 3$ & $5 \cdot 5$ & $16 \cdot 8$ & $0 \cdot 1$ & $(53 \cdot 3)$ & $(53 \cdot 3)$ \\
\hline Sweet products and substitutes & $51 \cdot 3$ & $50 \cdot 0$ & $34 \cdot 4$ & $(156 \cdot 0)$ & $(220 \cdot 0)$ & 104 & $96 \cdot 3$ & $53 \cdot 3$ & $49 \cdot 9$ & $36 \cdot 4$ & $(156 \cdot 0)$ & $(220 \cdot 0)$ \\
\hline Ice cream, ice lolly and substitutes & $24 \cdot 4$ & $42 \cdot 4$ & $0 \cdot 0$ & $(118 \cdot 0)$ & $(166 \cdot 7)$ & 40 & $37 \cdot 0$ & $66 \cdot 0$ & $46 \cdot 2$ & $55 \cdot 8$ & $(158 \cdot 3)$ & $(220 \cdot 0)$ \\
\hline Chocolate and substitutes & $7 \cdot 0$ & $10 \cdot 3$ & 0.0 & $(29 \cdot 4)$ & $(36 \cdot 7)$ & 48 & $44 \cdot 4$ & $15 \cdot 7$ & $10 \cdot 0$ & $13 \cdot 3$ & $(36 \cdot 7)$ & $(37 \cdot 6)$ \\
\hline Candies, jam and other sweet products (incl. sugar-free) & $2 \cdot 1$ & $4 \cdot 2$ & 0.0 & $(11 \cdot 7)$ & $(16 \cdot 0)$ & 31 & $28 \cdot 7$ & $7 \cdot 2$ & $5 \cdot 0$ & $7 \cdot 2$ & $(16 \cdot 0)$ & $(19 \cdot 4)$ \\
\hline Sugar, fructose, honey and other nutritious sweeteners & $15 \cdot 0$ & $20 \cdot 9$ & $11 \cdot 7$ & $(41 \cdot 8)$ & $(72 \cdot 8)$ & 81 & $75 \cdot 0$ & $20 \cdot 0$ & $22 \cdot 0$ & $16 \cdot 0$ & $(42 \cdot 0)$ & $(180 \cdot 2)$ \\
\hline Cocoa and cocoa-based powder & $2 \cdot 9$ & $10 \cdot 7$ & 0.0 & $(16 \cdot 3)$ & $(30 \cdot 0)$ & 21 & $19 \cdot 4$ & $14 \cdot 8$ & $20 \cdot 7$ & $11 \cdot 7$ & $(30 \cdot 0)$ & $(97 \cdot 9)$ \\
\hline Artificial sweeteners & 0.0 & $0 \cdot 1$ & 0.0 & $(0.0)$ & $(0.0)$ & 1 & 0.9 & $1 \cdot 0$ & - & $1 \cdot 0$ & $(1 \cdot 0)$ & $(1 \cdot 0)$ \\
\hline Meal substitutes & \multicolumn{2}{|c|}{ Not consumed } & & & & 0 & $0 \cdot 0$ & & & & & \\
\hline Water and other non-alcoholic beverages & $969 \cdot 3$ & $474 \cdot 4$ & $966 \cdot 9$ & $(1710 \cdot 0)$ & $(2871 \cdot 0)$ & 108 & $100 \cdot 0$ & $969 \cdot 3$ & $474 \cdot 4$ & $966 \cdot 9$ & $(1710 \cdot 0)$ & $(2871 \cdot 0)$ \\
\hline Tap water (as such, in beverages or recipes) & $228 \cdot 2$ & $289 \cdot 4$ & $94 \cdot 2$ & $(906 \cdot 7)$ & $(1150 \cdot 0)$ & 70 & $64 \cdot 8$ & $352 \cdot 1$ & $292 \cdot 7$ & $320 \cdot 0$ & $(960 \cdot 0)$ & $(1165 \cdot 0)$ \\
\hline Bottled water & $460 \cdot 6$ & $429 \cdot 3$ & $426 \cdot 7$ & $(1133 \cdot 3)$ & $(1466 \cdot 7)$ & 79 & $73 \cdot 1$ & $629 \cdot 7$ & $380 \cdot 8$ & $586 \cdot 7$ & $(1386 \cdot 7)$ & $(2060 \cdot 0)$ \\
\hline Coffee, tea, herbal tea and substitutes & $85 \cdot 0$ & $133 \cdot 1$ & $23 \cdot 3$ & $(350 \cdot 0)$ & $(559 \cdot 5)$ & 62 & $57 \cdot 4$ & $148 \cdot 0$ & $146 \cdot 9$ & $91 \cdot 3$ & $(493 \cdot 3)$ & $(651 \cdot 0)$ \\
\hline Fruit and vegetable juices & $97 \cdot 5$ & $158 \cdot 1$ & $8 \cdot 1$ & $(433 \cdot 3)$ & $(673 \cdot 0)$ & 81 & $75 \cdot 0$ & $130 \cdot 1$ & $170 \cdot 8$ & $68 \cdot 7$ & $(489 \cdot 7)$ & $(812 \cdot 7)$ \\
\hline Other soft drinks & $97 \cdot 9$ & $159 \cdot 6$ & 0.0 & $(413 \cdot 3)$ & $(586 \cdot 7)$ & 52 & $48 \cdot 1$ & $203 \cdot 4$ & $177 \cdot 6$ & $160 \cdot 0$ & $(550 \cdot 0)$ & $(1066 \cdot 7)$ \\
\hline Miscellaneous & $3 \cdot 8$ & $4 \cdot 3$ & $2 \cdot 4$ & $(12 \cdot 1)$ & $(19 \cdot 0)$ & 82 & $75 \cdot 9$ & $5 \cdot 0$ & $4 \cdot 3$ & $4 \cdot 1$ & $(12 \cdot 3)$ & $(20 \cdot 8)$ \\
\hline Total amount of foods and beveragest & $2273 \cdot 7$ & 683.6 & $2221 \cdot 2$ & $(3550 \cdot 1)$ & $(4771 \cdot 4)$ & 108 & $100 \cdot 0$ & $2273 \cdot 7$ & $683 \cdot 6$ & $2221 \cdot 2$ & $(3550 \cdot 1)$ & $(4771 \cdot 4)$ \\
\hline Total amount of liquid foods & $1143 \cdot 8$ & $469 \cdot 5$ & $1111 \cdot 1$ & $(1990 \cdot 0)$ & $(3055 \cdot 8)$ & 108 & $100 \cdot 0$ & $1143 \cdot 8$ & $469 \cdot 5$ & $1111 \cdot 1$ & $(1990 \cdot 0)$ & $(3055 \cdot 8)$ \\
\hline Total amount of solid foods & $1129 \cdot 9$ & $311 \cdot 1$ & $1091 \cdot 5$ & $(1762 \cdot 8)$ & $(2034 \cdot 8)$ & 108 & $100 \cdot 0$ & $1129 \cdot 9$ & $311 \cdot 1$ & $1091 \cdot 5$ & $(1762 \cdot 8)$ & $(2034 \cdot 8)$ \\
\hline
\end{tabular}

*High percentiles of consumption assessed on the basis of a $3 \mathrm{~d}$ survey provide an overestimate of long-term high levels of consumption. Values are enclosed in parentheses when the number of subjects to which they

refer is lower than 160 and 800 for the 95th and 99th percentile, respectively, since these values bear a large uncertainty and provide only a rough indication of high levels of consumption. 
17.9 years), females: Italian National Food Consumption Survey INRAN-SCAI 2005-06

\begin{tabular}{|c|c|c|c|c|c|c|c|c|c|c|c|c|}
\hline \multirow[b]{2}{*}{ Food categories } & \multicolumn{5}{|c|}{ Total population ( $n$ 139) } & \multicolumn{7}{|c|}{ Consumers } \\
\hline & Mean & SD & Median & 95th* & 99th* & $n$ & $\%$ & Mean & SD & Median & 95 th $^{*}$ & 99th* \\
\hline Cereals, cereal products and substitutes & $265 \cdot 3$ & $101 \cdot 2$ & $(260 \cdot 9)$ & $(417 \cdot 4)$ & $559 \cdot 6$ & 139 & $100 \cdot 0$ & $265 \cdot 3$ & $101 \cdot 2$ & $260 \cdot 9$ & $(417 \cdot 4)$ & $(559 \cdot 6)$ \\
\hline Bread & $85 \cdot 1$ & $64 \cdot 1$ & $(75 \cdot 0)$ & $(206 \cdot 5)$ & $240 \cdot 2$ & 126 & $90 \cdot 6$ & 93.9 & $60 \cdot \overline{9}$ & $80 \cdot 0$ & $(206 \cdot 5)$ & $(240 \cdot 2)$ \\
\hline Pasta and pasta substitutes & $56 \cdot 6$ & $30 \cdot 3$ & $(56 \cdot 0)$ & $(105 \cdot 3)$ & $133 \cdot 3$ & 129 & $92 \cdot 8$ & $61 \cdot 0$ & $26 \cdot 9$ & $59 \cdot 3$ & $(105 \cdot 3)$ & $(133 \cdot 3)$ \\
\hline Pizza & $14 \cdot 0$ & $35 \cdot 4$ & $(0 \cdot 0)$ & $(87 \cdot 5)$ & $183 \cdot 3$ & 34 & $24 \cdot 5$ & $57 \cdot 1$ & $52 \cdot 0$ & $33 \cdot 3$ & $(183 \cdot 3)$ & $(250 \cdot 0)$ \\
\hline Rice & $9 \cdot 7$ & $17 \cdot 0$ & $(0.0)$ & $(39 \cdot 5)$ & $67 \cdot 9$ & 43 & $30 \cdot 9$ & $31 \cdot 4$ & $15 \cdot 7$ & $30 \cdot 4$ & $(51 \cdot 3)$ & $(99 \cdot 4)$ \\
\hline Wheat, other cereals and flours & $43 \cdot 9$ & $39 \cdot 5$ & $(33 \cdot 8)$ & $(124 \cdot 3)$ & $140 \cdot 1$ & 128 & $92 \cdot 1$ & $47 \cdot 7$ & $38 \cdot 9$ & $42 \cdot 1$ & $(124 \cdot 3)$ & $(140 \cdot 1)$ \\
\hline Breakfast cereals & $1 \cdot 9$ & $5 \cdot 6$ & $(0 \cdot 0)$ & $(15 \cdot 0)$ & $30 \cdot 0$ & 22 & $15 \cdot 8$ & $11 \cdot 8$ & $9 \cdot 1$ & $8 \cdot 8$ & $(30 \cdot 0)$ & $(35 \cdot 0)$ \\
\hline Biscuits & $18 \cdot 8$ & $22 \cdot 8$ & $(12 \cdot 0)$ & $(66 \cdot 7)$ & $99 \cdot 3$ & 84 & $60 \cdot 4$ & $31 \cdot 2$ & $21 \cdot 7$ & $26 \cdot 7$ & $(76 \cdot 0)$ & $(103 \cdot 3)$ \\
\hline Savoury fine bakery products & $10 \cdot 6$ & $17 \cdot 7$ & $(0 \cdot 0)$ & $(45 \cdot 7)$ & $89 \cdot 5$ & 66 & $47 \cdot 5$ & $22 \cdot 4$ & $19 \cdot 9$ & $16 \cdot 7$ & $(66 \cdot 7)$ & $(104 \cdot 7)$ \\
\hline Cakes and sweet snacks & $24 \cdot 6$ & $31 \cdot 9$ & $(13 \cdot 3)$ & $(104 \cdot 0)$ & $125 \cdot 9$ & 86 & $61 \cdot 9$ & $39 \cdot 7$ & $32 \cdot 4$ & $28 \cdot 5$ & $(115 \cdot 2)$ & $(145 \cdot 8)$ \\
\hline Pulses, fresh and processed & 9.5 & $18 \cdot 4$ & $(0.0)$ & $(46 \cdot 3)$ & $83 \cdot 6$ & 42 & $30 \cdot 2$ & $31 \cdot 5$ & $20 \cdot 8$ & $23 \cdot 9$ & $(76.5)$ & $(91 \cdot 0)$ \\
\hline Vegetables, fresh and processed & $166 \cdot 4$ & $86 \cdot 3$ & (153.8) & $(331 \cdot 5)$ & $393 \cdot 3$ & 139 & $100 \cdot 0$ & $166 \cdot 4$ & $86 \cdot 3$ & $153 \cdot 8$ & $(331 \cdot 5)$ & (393.3) \\
\hline Leafy vegetables, fresh & $23 \cdot 5$ & $32 \cdot 6$ & $(13 \cdot 5)$ & $(93 \cdot 5)$ & $166 \cdot 7$ & 103 & $74 \cdot 1$ & $31 \cdot 7$ & $34 \cdot 3$ & $20 \cdot 0$ & $(105 \cdot 9)$ & $(166 \cdot 7)$ \\
\hline Tomatoes, fresh & $36 \cdot 0$ & $36 \cdot 5$ & $(25 \cdot 7)$ & $(113 \cdot 2)$ & $142 \cdot 0$ & 120 & $86 \cdot 3$ & $41 \cdot 7$ & $36 \cdot 1$ & $31 \cdot 1$ & $(115 \cdot 6)$ & $(142 \cdot 0)$ \\
\hline Other fruiting vegetables, fresh & $25 \cdot 8$ & $42 \cdot 8$ & $(2 \cdot 5)$ & $(117 \cdot 4)$ & $193 \cdot 0$ & 81 & $58 \cdot 3$ & $44 \cdot 3$ & $48 \cdot 2$ & $25 \cdot 0$ & $(122 \cdot 6)$ & $(205 \cdot 6)$ \\
\hline Roots and onions, fresh & $15 \cdot 0$ & $23 \cdot 5$ & $(7 \cdot 7)$ & $(55 \cdot 4)$ & $121 \cdot 3$ & 137 & $98 \cdot 6$ & $15 \cdot 2$ & $23 \cdot 6$ & $7 \cdot 8$ & $(55 \cdot 4)$ & $(121 \cdot 3)$ \\
\hline Other vegetables, fresh & $25 \cdot 7$ & $34 \cdot 1$ & $(11 \cdot 7)$ & $(91 \cdot 8)$ & $180 \cdot 0$ & 113 & $81 \cdot 3$ & $31 \cdot 6$ & $35 \cdot 2$ & $22 \cdot 5$ & $(92 \cdot 8)$ & $(180 \cdot 0)$ \\
\hline Vegetables, processed & $38 \cdot 5$ & $34 \cdot 5$ & $(33.4)$ & $(100 \cdot 2)$ & $136 \cdot 8$ & 106 & $76 \cdot 3$ & $50 \cdot 5$ & $30 \cdot \overline{9}$ & $49 \cdot 7$ & $(107 \cdot 5)$ & $(136 \cdot 8)$ \\
\hline Spices and herbs & $2 \cdot 0$ & $4 \cdot 0$ & $(1 \cdot 0)$ & $(6 \cdot 5)$ & $27 \cdot 1$ & 109 & $78 \cdot 4$ & $2 \cdot 5$ & $4 \cdot 4$ & $1 \cdot 4$ & $(7 \cdot 1)$ & $(27 \cdot 1)$ \\
\hline Potatoes, tubers and their products & $53 \cdot 7$ & $53 \cdot 6$ & $(49 \cdot 0)$ & $(161 \cdot 4)$ & $191 \cdot 5$ & 96 & $69 \cdot 1$ & $77 \cdot 8$ & $47 \cdot 8$ & $73 \cdot 4$ & $(167 \cdot 3)$ & (193.9) \\
\hline Fruit, fresh and processed & $178 \cdot 5$ & $132 \cdot 0$ & $(182 \cdot 0)$ & $(428 \cdot 8)$ & $590 \cdot 7$ & 122 & $87 \cdot 8$ & $203 \cdot 4$ & $121 \cdot 6$ & $200 \cdot 8$ & $(428 \cdot 8)$ & $(590 \cdot 7)$ \\
\hline Citrus fruit, fresh & $30 \cdot 4$ & $66 \cdot 3$ & $(0 \cdot 0)$ & $(182 \cdot 0)$ & $298 \cdot 0$ & 59 & $42 \cdot 4$ & $71 \cdot 7$ & $86 \cdot 4$ & $60 \cdot 7$ & $(274 \cdot 2)$ & $(381 \cdot 0)$ \\
\hline Exotic fruit, fresh & $23 \cdot 4$ & $36 \cdot 1$ & $(0 \cdot 0)$ & $(100 \cdot 0)$ & $171 \cdot 7$ & 61 & $43 \cdot 9$ & $53 \cdot 3$ & $37 \cdot 0$ & $33 \cdot 3$ & $(120 \cdot 5)$ & $(182 \cdot 8)$ \\
\hline Other fruit, fresh & $122 \cdot 5$ & $123 \cdot 0$ & $(82 \cdot 7)$ & $(374 \cdot 0)$ & $488 \cdot 3$ & 105 & $75 \cdot 5$ & $162 \cdot 2$ & $116 \cdot 5$ & $150 \cdot 0$ & $(389 \cdot 3)$ & $(488 \cdot 3)$ \\
\hline Nuts, seeds, olives and their products, dried fruit & 1.9 & $3 \cdot 7$ & $(0 \cdot 0)$ & $(10 \cdot 7)$ & $13 \cdot 3$ & 37 & $26 \cdot 6$ & $7 \cdot 0$ & $4 \cdot 0$ & $6 \cdot 3$ & $(13 \cdot 3)$ & $(20 \cdot 8)$ \\
\hline Other processed fruit (in syrup, in purée, etc.) & $0 \cdot 2$ & $2 \cdot 8$ & $(0 \cdot 0)$ & $(0 \cdot 0)$ & $0 \cdot 0$ & 1 & 0.7 & $33 \cdot 3$ & - & $33 \cdot 3$ & $(33 \cdot 3)$ & $(33 \cdot 3)$ \\
\hline Meat, meat products and substitutes & $107 \cdot 4$ & $47 \cdot 3$ & $(107 \cdot 4)$ & (193.8) & $221 \cdot 4$ & 139 & $100 \cdot 0$ & $107 \cdot 4$ & $47 \cdot 3$ & $107 \cdot 4$ & (193.8) & $(221 \cdot 4)$ \\
\hline Beef and veal, not preserved, excl. offal & $41 \cdot 8$ & $35 \cdot 8$ & $(36 \cdot 0)$ & $(114 \cdot 0)$ & $129 \cdot 6$ & 110 & $79 \cdot 1$ & $52 \cdot 8$ & $32 \cdot 2$ & 43.9 & $(117 \cdot 6)$ & $(129 \cdot 6)$ \\
\hline Pork, not preserved, excl. offal & $13 \cdot 3$ & $24 \cdot 5$ & $(0 \cdot 0)$ & $(73.9)$ & $105 \cdot 5$ & 46 & $33 \cdot 1$ & $40 \cdot 2$ & $27 \cdot 2$ & $35 \cdot 2$ & $(93 \cdot 8)$ & $(105 \cdot 8)$ \\
\hline Poultry and game, not preserved, excl. offal & $18 \cdot 5$ & $23 \cdot 4$ & $(0 \cdot 0)$ & $(55 \cdot 3)$ & $90 \cdot 1$ & 60 & $43 \cdot 2$ & $42 \cdot \overline{9}$ & $14 \cdot \overline{5}$ & $41 \cdot 0$ & $(70 \cdot 3)$ & $(90 \cdot 4)$ \\
\hline Other meats, not preserved, excl. offal & $4 \cdot 4$ & $14 \cdot 6$ & $(0.0)$ & $(47 \cdot 5)$ & $66 \cdot 7$ & 13 & $9 \cdot 4$ & $47 \cdot 2$ & $16 \cdot 0$ & $47 \cdot 5$ & $(80 \cdot 3)$ & $(80 \cdot 3)$ \\
\hline $\begin{array}{l}\text { Ham, salami, sausages and other preserved meats, } \\
\text { excl. offal }\end{array}$ & $28 \cdot 6$ & $25 \cdot 8$ & $(21 \cdot 7)$ & $(72 \cdot 1)$ & $109 \cdot 4$ & 118 & $84 \cdot 9$ & $33 \cdot 7$ & $24 \cdot 8$ & $25 \cdot 5$ & $(85 \cdot 7)$ & $(109 \cdot 4)$ \\
\hline Offal, blood and their products & 0.7 & $5 \cdot 5$ & $(0 \cdot 0)$ & $(0 \cdot 0)$ & $33 \cdot 3$ & 4 & $2 \cdot 9$ & $25 \cdot 9$ & $22 \cdot 9$ & $20 \cdot 5$ & $(55 \cdot 1)$ & $(55 \cdot 1)$ \\
\hline Meat substitutes & Not co & sumed & & & & 0 & 0.0 & & & & & \\
\hline Fish, seafood and their products & $49 \cdot 4$ & $57 \cdot 1$ & $(37 \cdot 5)$ & $(173 \cdot 6)$ & $255 \cdot 7$ & 101 & $72 \cdot 7$ & $68 \cdot 0$ & $56 \cdot 8$ & $53 \cdot 3$ & $(181 \cdot 8)$ & $(255 \cdot 7)$ \\
\hline Fish and seafood, fresh and frozen & $44 \cdot 4$ & $57 \cdot 8$ & $(27 \cdot 2)$ & $(170 \cdot 2)$ & $255 \cdot 7$ & 81 & $58 \cdot 3$ & $76 \cdot 2$ & $57 \cdot 5$ & $57 \cdot 5$ & $(181 \cdot 8)$ & $(255 \cdot 7)$ \\
\hline Fish and seafood, preserved & $5 \cdot 0$ & $11 \cdot 2$ & $(0 \cdot 0)$ & $(23 \cdot 5)$ & $58 \cdot 4$ & 47 & $33 \cdot 8$ & $14 \cdot 8$ & $15 \cdot 1$ & $9 \cdot 7$ & $(41 \cdot 3)$ & $(73 \cdot 0)$ \\
\hline Milk, milk products and substitutes & $215 \cdot 7$ & $105 \cdot 3$ & $(208.5)$ & $(416 \cdot 8)$ & $470 \cdot 2$ & 137 & $98 \cdot 6$ & 218.9 & $102 \cdot 7$ & $209 \cdot 6$ & $(416 \cdot 8)$ & $(470 \cdot 2)$ \\
\hline $\begin{array}{l}\text { Milk, milk-based beverages, human milk, infant } \\
\text { formula and substitutes }\end{array}$ & $139 \cdot 8$ & $97 \cdot 5$ & $(150 \cdot 0)$ & $(264 \cdot 7)$ & $343 \cdot 8$ & 116 & $83 \cdot 5$ & $167 \cdot 5$ & $82 \cdot 0$ & $166 \cdot 7$ & $(264 \cdot 9)$ & $(343 \cdot 8)$ \\
\hline Yoghurt and fermented milk & $20 \cdot 8$ & $46 \cdot 7$ & $(0 \cdot 0)$ & $(125 \cdot 0)$ & $208 \cdot 3$ & 33 & $23 \cdot 7$ & $87 \cdot 7$ & $58 \cdot 2$ & $52 \cdot 0$ & $(208 \cdot 3)$ & $(250 \cdot 0)$ \\
\hline Cheese and substitutes & $54 \cdot 6$ & $38 \cdot 1$ & $(46.9)$ & $(127 \cdot 6)$ & $150 \cdot 3$ & 135 & $97 \cdot 1$ & $56 \cdot 2$ & $37 \cdot 4$ & $47 \cdot 5$ & $(127 \cdot 6)$ & $(150 \cdot 3)$ \\
\hline Milk-based desserts and substitutes & 0.5 & $3 \cdot 4$ & $(0.0)$ & $(0 \cdot 0)$ & $16 \cdot 7$ & 5 & 3.6 & $15 \cdot 1$ & $10 \cdot 9$ & $10 \cdot 4$ & $(33 \cdot 3)$ & $(33 \cdot 3)$ \\
\hline
\end{tabular}




\begin{tabular}{|c|c|c|c|c|c|c|c|c|c|c|c|c|}
\hline \multirow[b]{2}{*}{ Food categories } & \multicolumn{5}{|c|}{ Total population ( $n$ 139) } & \multicolumn{7}{|c|}{ Consumers } \\
\hline & Mean & SD & Median & 95th* & 99th* & $n$ & $\%$ & Mean & SD & Median & 95th* & 99th* \\
\hline Oils and fats & $38 \cdot 8$ & $16 \cdot 1$ & $(36 \cdot 2)$ & $(61 \cdot 7)$ & $97 \cdot 6$ & 138 & $99 \cdot 3$ & $39 \cdot 1$ & $15 \cdot 8$ & $36 \cdot 2$ & $(61 \cdot 7)$ & $(97 \cdot 6)$ \\
\hline Olive oil & $29 \cdot 5$ & $11 \cdot 7$ & $(28 \cdot 8)$ & $(53 \cdot 4)$ & $59 \cdot 7$ & 138 & $99 \cdot 3$ & $29 \cdot 7$ & $11 \cdot 5$ & $28 \cdot 9$ & $(53 \cdot 4)$ & $(59 \cdot 7)$ \\
\hline Other vegetable oils & $3 \cdot 3$ & $3 \cdot 7$ & $(3 \cdot 0)$ & $(10 \cdot 3)$ & $13 \cdot 5$ & 81 & $58 \cdot 3$ & $5 \cdot 7$ & $3 \cdot 1$ & $5 \cdot 2$ & $(11.9)$ & $(19 \cdot 5)$ \\
\hline Butter and creams & $4 \cdot 7$ & $10 \cdot 3$ & $(0.0)$ & $(23 \cdot 0)$ & $62 \cdot 5$ & 62 & $44 \cdot 6$ & $10 \cdot 5$ & $13 \cdot 3$ & 4.9 & $(25 \cdot 0)$ & $(76 \cdot 8)$ \\
\hline Other fats & $1 \cdot 3$ & $2 \cdot 9$ & $(0.0)$ & $(8 \cdot 7)$ & $12 \cdot 2$ & 35 & $25 \cdot 2$ & $5 \cdot 1$ & $3 \cdot 7$ & $4 \cdot 0$ & $(12 \cdot 2)$ & (13.7) \\
\hline Eggs & $20 \cdot 8$ & $24 \cdot 2$ & $(12 \cdot 1)$ & $(72 \cdot 5)$ & $115 \cdot 2$ & 111 & $79 \cdot 9$ & $26 \cdot 1$ & $24 \cdot 4$ & $18 \cdot 0$ & $(76 \cdot 3)$ & $(115 \cdot 2)$ \\
\hline Alcoholic beverages and substitutes & $1 \cdot 3$ & $8 \cdot 0$ & 0.0 & $(0.3)$ & $(55 \cdot 1)$ & 82 & $59 \cdot 0$ & $2 \cdot 2$ & $10 \cdot 4$ & $0 \cdot 1$ & $(3 \cdot 5)$ & $(66 \cdot 7)$ \\
\hline Regular wine and substitutes & $0 \cdot 0$ & $0 \cdot 1$ & $0 \cdot 0$ & $(0 \cdot 2)$ & $(0 \cdot 2)$ & 78 & $56 \cdot 1$ & $0 \cdot 1$ & $0 \cdot 1$ & $0 \cdot 1$ & $(0 \cdot 2)$ & $(0 \cdot 2)$ \\
\hline Beer, cider and substitutes & $0 \cdot 9$ & $7 \cdot 3$ & $0 \cdot 0$ & $(0 \cdot 0)$ & $(55 \cdot 0)$ & 3 & $2 \cdot 2$ & $40 \cdot 6$ & $35 \cdot 6$ & $55 \cdot 0$ & $(66 \cdot 7)$ & $(66 \cdot 7)$ \\
\hline $\begin{array}{l}\text { Sweet wine, spumante, wine-based appetizers, } \\
\text { spirits and liquors }\end{array}$ & $0 \cdot 4$ & $3 \cdot 5$ & $0 \cdot 0$ & $(0 \cdot 1)$ & $(7 \cdot 0)$ & 17 & $12 \cdot 2$ & $3 \cdot 1$ & $9 \cdot 7$ & $0 \cdot 1$ & $(40 \cdot 0)$ & $(40 \cdot 0)$ \\
\hline Sweet products and substitutes & $36 \cdot 4$ & $35 \cdot 3$ & $28 \cdot 4$ & $(100 \cdot 7)$ & $(166 \cdot 9)$ & 129 & $92 \cdot 8$ & $39 \cdot 3$ & $35 \cdot 1$ & $30 \cdot 8$ & $(100 \cdot 7)$ & $(166 \cdot 9)$ \\
\hline Ice cream, ice lolly and substitutes & $15 \cdot 8$ & $32 \cdot 3$ & 0.0 & $(80 \cdot 0)$ & $(156 \cdot 7)$ & 42 & $30 \cdot 2$ & $52 \cdot 4$ & $39 \cdot 3$ & $33 \cdot 3$ & $(110 \cdot 7)$ & $(216 \cdot 7)$ \\
\hline Chocolate and substitutes & $6 \cdot 5$ & $10 \cdot 7$ & 0.0 & $(34 \cdot 3)$ & $(40 \cdot 0)$ & 56 & $40 \cdot 3$ & $16 \cdot 1$ & $11 \cdot 3$ & $13 \cdot 3$ & $(40 \cdot 0)$ & $(43 \cdot 3)$ \\
\hline Candies, jam and other sweet products (incl. sugar-free) & $2 \cdot 3$ & $5 \cdot 1$ & $0 \cdot 0$ & $(13 \cdot 3)$ & $(17 \cdot 0)$ & 49 & $35 \cdot 3$ & $6 \cdot 5$ & $6 \cdot 9$ & $4 \cdot 0$ & $(17 \cdot 0)$ & $(36 \cdot 4)$ \\
\hline Sugar, fructose, honey and other nutritious sweeteners & $9 \cdot 9$ & $10 \cdot 4$ & $8 \cdot 0$ & $(31 \cdot 6)$ & $(44 \cdot 3)$ & 101 & $72 \cdot 7$ & $13 \cdot 7$ & $9 \cdot 9$ & $11 \cdot 8$ & $(31 \cdot 8)$ & $(44 \cdot 3)$ \\
\hline Cocoa and cocoa-based powder & $1 \cdot 9$ & $5 \cdot 1$ & $0 \cdot 0$ & $(14 \cdot 0)$ & $(24 \cdot 7)$ & 30 & $21 \cdot 6$ & $8 \cdot 7$ & $7 \cdot 7$ & $7 \cdot 0$ & $(24 \cdot 7)$ & $(36 \cdot 1)$ \\
\hline Artificial sweeteners & $0 \cdot 0$ & $0 \cdot 0$ & $0 \cdot 0$ & $(0 \cdot 0)$ & $(0 \cdot 0)$ & 1 & $0 \cdot 7$ & $0 \cdot 0$ & - & $0 \cdot 0$ & $(0 \cdot 0)$ & $(0 \cdot 0)$ \\
\hline Meal substitutes & \multicolumn{2}{|c|}{ Not consumed } & & & & 0 & $0 \cdot 0$ & & & & & \\
\hline Water and other non-alcoholic beverages & $777 \cdot 8$ & $340 \cdot 4$ & $737 \cdot 1$ & $(1466 \cdot 7)$ & $(1905 \cdot 0)$ & 139 & $100 \cdot 0$ & $777 \cdot 8$ & $340 \cdot 4$ & $737 \cdot 1$ & $(1466 \cdot 7)$ & $(1905 \cdot 0)$ \\
\hline Tap water (as such, in beverages or recipes) & $208 \cdot 1$ & $326 \cdot 6$ & $53 \cdot 3$ & $(826 \cdot 7)$ & $(1773 \cdot 3)$ & 84 & $60 \cdot 4$ & $344 \cdot 4$ & $360 \cdot 4$ & $277 \cdot 5$ & $(906 \cdot 7)$ & $(1813 \cdot 3)$ \\
\hline Bottled water & $389 \cdot 6$ & $332 \cdot 8$ & $386 \cdot 7$ & $(906 \cdot 7)$ & $(1506 \cdot 7)$ & 102 & $73 \cdot 4$ & $531 \cdot 0$ & $274 \cdot 8$ & $533 \cdot 3$ & $(913 \cdot 3)$ & $(1506 \cdot 7)$ \\
\hline Coffee, tea, herbal tea and substitutes & $54 \cdot 0$ & $88 \cdot 5$ & $15 \cdot 0$ & $(250 \cdot 0)$ & $(480 \cdot 0)$ & 79 & $56 \cdot 8$ & $95 \cdot 1$ & $99 \cdot 6$ & $66 \cdot 7$ & $(266 \cdot 7)$ & $(532 \cdot 1)$ \\
\hline Fruit and vegetable juices & $71 \cdot 7$ & $103 \cdot 6$ & $5 \cdot 3$ & $(316 \cdot 7)$ & $(416 \cdot 7)$ & 94 & $67 \cdot 6$ & $106 \cdot 0$ & $110 \cdot 7$ & $68 \cdot 9$ & $(333 \cdot 3)$ & $(469 \cdot 4)$ \\
\hline Other soft drinks & $54 \cdot 3$ & $91 \cdot 1$ & 0.0 & $(266 \cdot 7)$ & $(403 \cdot 3)$ & 57 & $41 \cdot 0$ & $132 \cdot 4$ & $99 \cdot 8$ & $110 \cdot 0$ & $(340 \cdot 0)$ & $(533 \cdot 3)$ \\
\hline Miscellaneous & $2 \cdot 7$ & $2 \cdot 9$ & $1 \cdot 8$ & $(8 \cdot 6)$ & $(12 \cdot 3)$ & 107 & $77 \cdot 0$ & $3 \cdot 5$ & $2 \cdot 8$ & $2 \cdot 6$ & $(9 \cdot 5)$ & $(12 \cdot 3)$ \\
\hline Total amount of foods and beveragest & 1923.9 & $520 \cdot 0$ & $1950 \cdot 4$ & $(2907 \cdot 7)$ & $(3169 \cdot 5)$ & 139 & $100 \cdot 0$ & $1923 \cdot 9$ & $520 \cdot 0$ & $1950 \cdot 4$ & $(2907 \cdot 7)$ & $(3169 \cdot 5)$ \\
\hline Total amount of liquid foods & $920 \cdot 4$ & $339 \cdot 4$ & $895 \cdot 0$ & $(1552 \cdot 4)$ & $(1983 \cdot 5)$ & 139 & $100 \cdot 0$ & $920 \cdot 4$ & $339 \cdot 4$ & $895 \cdot 0$ & $(1552 \cdot 4)$ & $(1983 \cdot 5)$ \\
\hline Total amount of solid foods & $1003 \cdot 4$ & $276 \cdot 6$ & $978 \cdot 7$ & $(1536 \cdot 0)$ & $(1716 \cdot 4)$ & 139 & $100 \cdot 0$ & $1003 \cdot 4$ & $276 \cdot 6$ & $978 \cdot 7$ & $(1536 \cdot 0)$ & $(1716 \cdot 4)$ \\
\hline
\end{tabular}

*High percentiles of consumption assessed on the basis of a $3 \mathrm{~d}$ survey provide an overestimate of long-term high levels of consumption. Values are enclosed in parentheses when the number of subjects to which they

refer is lower than 160 and 800 for the 95th and 99th percentile, respectively, since these values bear a large uncertainty and provide only a rough indication of high levels of consumption. 
Table 8 Mean, standard deviation, median and high percentiles of individual daily consumption ( $3 \mathrm{~d}$ average) by food category in the total population and in consumers ( $\mathrm{g} / \mathrm{d}$ ) - adults ( 18 to $64 \cdot 9$ years), males: Italian National Food Consumption Survey INRAN-SCAI 2005-06

\begin{tabular}{|c|c|c|c|c|c|c|c|c|c|c|c|c|}
\hline \multirow[b]{2}{*}{ Food categories } & \multicolumn{5}{|c|}{ Total population ( $n$ 1068) } & \multicolumn{7}{|c|}{ Consumers } \\
\hline & Mean & SD & Median & $95 \mathrm{th}^{*}$ & 99th* & $n$ & $\%$ & Mean & SD & Median & 95th* & 99th* \\
\hline Cereals, cereal products and substitutes & $295 \cdot 7$ & $110 \cdot 4$ & $288 \cdot 1$ & $486 \cdot 9$ & $611 \cdot 7$ & 1068 & $100 \cdot 0$ & $295 \cdot 7$ & $110 \cdot 4$ & $288 \cdot 1$ & $486 \cdot 9$ & $611 \cdot 7$ \\
\hline Bread & $127 \cdot 2$ & $84 \cdot 7$ & $115 \cdot 0$ & $285 \cdot 0$ & $375 \cdot 0$ & 1015 & $95 \cdot 0$ & $133 \cdot 8$ & $81 \cdot 6$ & $120 \cdot 0$ & $290 \cdot 0$ & $375 \cdot 0$ \\
\hline Pasta and pasta substitutes & $60 \cdot 3$ & $35 \cdot 3$ & $59 \cdot 3$ & $118 \cdot 4$ & $156 \cdot 1$ & 976 & $91 \cdot 4$ & $66 \cdot 0$ & $31 \cdot 4$ & $62 \cdot 7$ & $121 \cdot 6$ & $156 \cdot 9$ \\
\hline Pizza & $8 \cdot 7$ & $27 \cdot 3$ & 0.0 & $66 \cdot 7$ & $133 \cdot 3$ & 146 & $13 \cdot 7$ & $63 \cdot 6$ & $44 \cdot 4$ & $50 \cdot 0$ & $(150 \cdot 0)$ & $(250 \cdot 0)$ \\
\hline Rice & $16 \cdot 4$ & $25 \cdot 9$ & $0 \cdot 0$ & $67 \cdot 9$ & $110 \cdot 0$ & 426 & $39 \cdot 9$ & $41 \cdot 2$ & $25 \cdot 7$ & $34 \cdot 2$ & $83 \cdot 3$ & $(122 \cdot 9)$ \\
\hline Wheat, other cereals and flours & $44 \cdot 1$ & $40 \cdot 8$ & $35 \cdot 9$ & $120 \cdot 1$ & $165 \cdot 1$ & 922 & $86 \cdot 3$ & $51 \cdot 1$ & $39 \cdot 7$ & $45 \cdot 2$ & $123 \cdot 7$ & $166 \cdot 0$ \\
\hline Breakfast cereals & 0.9 & $4 \cdot 7$ & 0.0 & $4 \cdot 7$ & $30 \cdot 0$ & 62 & $5 \cdot 8$ & $16 \cdot 1$ & $12 \cdot 1$ & $14 \cdot 0$ & $(40 \cdot 0)$ & $(50 \cdot 0)$ \\
\hline Biscuits & $13 \cdot 4$ & $23 \cdot 5$ & $0 \cdot 0$ & $56 \cdot 9$ & $103 \cdot 3$ & 467 & $43 \cdot 7$ & $30 \cdot 6$ & $27 \cdot 0$ & $24 \cdot 0$ & $83 \cdot 3$ & $(140 \cdot 0)$ \\
\hline Savoury fine bakery products & $5 \cdot 7$ & $14 \cdot 5$ & $0 \cdot 0$ & $32 \cdot 0$ & 63.0 & 284 & $26 \cdot 6$ & $21 \cdot 6$ & $21 \cdot 3$ & $16 \cdot 0$ & $50 \cdot 0$ & $(146 \cdot 3)$ \\
\hline Cakes and sweet snacks & $19 \cdot 0$ & $31 \cdot 2$ & $0 \cdot 0$ & $80 \cdot 0$ & $149 \cdot 4$ & 501 & $46 \cdot 9$ & $40 \cdot 4$ & $34 \cdot 8$ & $31 \cdot 8$ & $114 \cdot 0$ & $(162 \cdot 1)$ \\
\hline Pulses, fresh and processed & $11 \cdot 7$ & $24 \cdot 7$ & $0 \cdot 0$ & $60 \cdot 4$ & $97 \cdot 9$ & 358 & $33 \cdot 5$ & $34 \cdot 8$ & 31.9 & $24 \cdot 9$ & $87 \cdot 4$ & $(126 \cdot 7)$ \\
\hline Vegetables, fresh and processed & $232 \cdot 6$ & $120 \cdot 4$ & $213 \cdot 4$ & $442 \cdot 1$ & $618 \cdot 9$ & 1067 & $99 \cdot 9$ & $232 \cdot 9$ & $120 \cdot 2$ & $213 \cdot 5$ & $442 \cdot 1$ & 618.9 \\
\hline Leafy vegetables, fresh & $45 \cdot 3$ & 53.9 & $30 \cdot 1$ & $146 \cdot 1$ & $247 \cdot 8$ & 913 & $85 \cdot 5$ & $53 \cdot 0$ & $54 \cdot 7$ & $36 \cdot 4$ & $151 \cdot 8$ & $247 \cdot 8$ \\
\hline Tomatoes, fresh & $48 \cdot 4$ & $47 \cdot 3$ & $37 \cdot 7$ & $140 \cdot 5$ & $219 \cdot 0$ & 927 & $86 \cdot 8$ & $55 \cdot 8$ & $46 \cdot 5$ & $45 \cdot 2$ & $145 \cdot 8$ & $223 \cdot 2$ \\
\hline Other fruiting vegetables, fresh & $34 \cdot 0$ & $54 \cdot 9$ & $6 \cdot 7$ & $145 \cdot 0$ & $235 \cdot 5$ & 687 & $64 \cdot 3$ & $52 \cdot 9$ & $60 \cdot 8$ & $32 \cdot 4$ & $165 \cdot 8$ & $(269 \cdot 6)$ \\
\hline Roots and onions, fresh & $20 \cdot 0$ & $27 \cdot 0$ & $10 \cdot 0$ & $78 \cdot 4$ & $131 \cdot 2$ & 1052 & $98 \cdot 5$ & $20 \cdot 3$ & $27 \cdot 1$ & $10 \cdot 4$ & 78.9 & $131 \cdot 2$ \\
\hline Other vegetables, fresh & $41 \cdot 2$ & $50 \cdot 7$ & $21 \cdot 9$ & $143 \cdot 0$ & $225 \cdot 0$ & 882 & $82 \cdot 6$ & $49 \cdot 9$ & $51 \cdot 7$ & $34 \cdot 3$ & $149 \cdot 3$ & $238 \cdot 0$ \\
\hline Vegetables, processed & $41 \cdot \overline{6}$ & $35 \cdot 7$ & $37 \cdot 8$ & $103 \cdot 6$ & $140 \cdot 2$ & 855 & $80 \cdot 1$ & $52 \cdot 0$ & $32 \cdot 4$ & $42 \cdot 8$ & $111 \cdot 6$ & $158 \cdot 3$ \\
\hline Spices and herbs & $2 \cdot 0$ & $2 \cdot 8$ & $1 \cdot 2$ & $6 \cdot 4$ & $11 \cdot 6$ & 914 & $85 \cdot 6$ & $2 \cdot 4$ & $2 \cdot 9$ & 1.5 & $6 \cdot 8$ & $11 \cdot 6$ \\
\hline Potatoes, tubers and their products & $54 \cdot 3$ & $57 \cdot 4$ & $48 \cdot 1$ & $162 \cdot 9$ & $229 \cdot 2$ & 738 & $69 \cdot 1$ & $78 \cdot 5$ & 53.5 & $73 \cdot 2$ & $179 \cdot 0$ & $(240.5)$ \\
\hline Fruit, fresh and processed & $200 \cdot 0$ & $161 \cdot 7$ & $172 \cdot 7$ & $507 \cdot 3$ & $735 \cdot 3$ & 995 & 93.2 & $214 \cdot 7$ & $157 \cdot 8$ & 184.9 & $517 \cdot 7$ & 747.8 \\
\hline Citrus fruit, fresh & $48 \cdot 4$ & $98 \cdot 7$ & $0 \cdot 0$ & $242 \cdot 7$ & $426 \cdot 7$ & 478 & $44 \cdot 8$ & $108 \cdot 3$ & $123 \cdot 8$ & $65 \cdot 8$ & $332 \cdot 7$ & $(604 \cdot 7)$ \\
\hline Exotic fruit, fresh & $16 \cdot 7$ & $30 \cdot 5$ & $0 \cdot 0$ & $83 \cdot 3$ & $138 \cdot 0$ & 402 & $37 \cdot 6$ & $44 \cdot 3$ & $35 \cdot 3$ & $33 \cdot 3$ & $107 \cdot 5$ & $(166 \cdot 7)$ \\
\hline Other fruit, fresh & $131 \cdot 7$ & $133 \cdot 0$ & $100 \cdot 0$ & $384 \cdot 3$ & $566 \cdot 7$ & 851 & $79 \cdot 7$ & $165 \cdot 3$ & $129 \cdot 1$ & $138 \cdot 3$ & $408 \cdot 3$ & $632 \cdot 4$ \\
\hline Nuts, seeds, olives and their products, dried fruit & $3 \cdot 1$ & $6 \cdot 8$ & $0 \cdot 0$ & $14 \cdot 8$ & $27 \cdot 5$ & 342 & $32 \cdot 0$ & $9 \cdot 6$ & $9 \cdot 0$ & $7 \cdot 5$ & $23 \cdot 5$ & $(42 \cdot 7)$ \\
\hline Other processed fruit (in syrup, in purée, etc.) & $0 \cdot 1$ & $1 \cdot 8$ & 0.0 & 0.0 & 0.6 & 12 & $1 \cdot 1$ & $8 \cdot 1$ & $15 \cdot 3$ & 0.9 & $(50 \cdot 0)$ & $(50 \cdot 0)$ \\
\hline Meat, meat products and substitutes & $131 \cdot 5$ & 73.5 & $120 \cdot 6$ & $259 \cdot 0$ & $357 \cdot 9$ & 1064 & $99 \cdot 6$ & $132 \cdot 0$ & $73 \cdot 2$ & $121 \cdot 0$ & $259 \cdot 0$ & $357 \cdot 9$ \\
\hline Beef and veal, not preserved, excl. offal & $48 \cdot 7$ & $47 \cdot 3$ & $41 \cdot 2$ & $137 \cdot 4$ & $215 \cdot 9$ & 814 & $76 \cdot 2$ & 63.9 & $44 \cdot 3$ & $53 \cdot 8$ & $146 \cdot 7$ & $233 \cdot 4$ \\
\hline Pork, not preserved, excl. offal & $16 \cdot 4$ & $30 \cdot 8$ & 0.0 & $70 \cdot 6$ & $125 \cdot 9$ & 367 & $34 \cdot \overline{4}$ & $47 \cdot 7$ & $35 \cdot 5$ & $46 \cdot 7$ & $101 \cdot 5$ & $(177 \cdot 4)$ \\
\hline Poultry and game, not preserved, excl. offal & $22 \cdot 8$ & $33 \cdot 1$ & 0.0 & $90 \cdot 1$ & $136 \cdot 8$ & 448 & 41.9 & $54 \cdot 4$ & $29 \cdot 9$ & $48 \cdot 2$ & $111 \cdot 3$ & $(166 \cdot 7)$ \\
\hline Other meats, not preserved, excl. offal & $6 \cdot 8$ & $23 \cdot 6$ & $0 \cdot 0$ & $52 \cdot 0$ & $106 \cdot 7$ & 127 & $11 \cdot 9$ & $57 \cdot 2$ & $42 \cdot 5$ & $49 \cdot 4$ & $(119 \cdot 3)$ & $(135 \cdot 8)$ \\
\hline $\begin{array}{l}\text { Ham, salami, sausages and other preserved meats, } \\
\text { excl. offal }\end{array}$ & $35 \cdot 4$ & $32 \cdot 0$ & $27 \cdot 9$ & $94 \cdot 1$ & $139 \cdot 6$ & 931 & $87 \cdot 2$ & $40 \cdot 7$ & $31 \cdot 0$ & $33 \cdot 3$ & 96.9 & $139 \cdot 8$ \\
\hline Offal, blood and their products & $1 \cdot 3$ & $8 \cdot 2$ & 0.0 & 0.0 & $49 \cdot 4$ & 43 & $4 \cdot 0$ & $31 \cdot 8$ & $26 \cdot 7$ & $25 \cdot 0$ & $(65 \cdot 8)$ & $(140 \cdot 3)$ \\
\hline Meat substitutes & $0 \cdot 0$ & $1 \cdot 0$ & $0 \cdot 0$ & $0 \cdot 0$ & $0 \cdot 0$ & 1 & $0 \cdot 1$ & $33 \cdot 3$ & - & $33 \cdot 3$ & $(33 \cdot 3)$ & $(33 \cdot 3)$ \\
\hline Fish, seafood and their products & 48.5 & $55 \cdot 6$ & $34 \cdot 7$ & $160 \cdot 0$ & $244 \cdot 3$ & 750 & $70 \cdot 2$ & $69 \cdot 0$ & $54 \cdot 6$ & 58.4 & $182 \cdot 3$ & $(254 \cdot 6)$ \\
\hline Fish and seafood, fresh and frozen & $41 \cdot 2$ & $54 \cdot 5$ & $6 \cdot 2$ & $152 \cdot 1$ & $226 \cdot 9$ & 587 & $55 \cdot 0$ & $75 \cdot 0$ & $53 \cdot 6$ & $62 \cdot 5$ & $185 \cdot 4$ & $(252 \cdot 0)$ \\
\hline Fish and seafood, preserved & $7 \cdot 2$ & $13 \cdot 8$ & $0 \cdot 0$ & $34 \cdot 5$ & $66 \cdot 2$ & 401 & $37 \cdot 5$ & $19 \cdot 3$ & $16 \cdot 5$ & $14 \cdot 2$ & $58 \cdot 4$ & $(73.0)$ \\
\hline Milk, milk products and substitutes & $177 \cdot 5$ & $126 \cdot 4$ & $157 \cdot 0$ & $381 \cdot 0$ & $556 \cdot 3$ & 1062 & $99 \cdot 4$ & 178.5 & $126 \cdot 0$ & $158 \cdot 2$ & $381 \cdot 0$ & $556 \cdot 3$ \\
\hline $\begin{array}{l}\text { Milk, milk-based beverages, human milk, infant formula } \\
\text { and substitutes }\end{array}$ & $94 \cdot 3$ & $107 \cdot 9$ & $52 \cdot 0$ & $255 \cdot 8$ & $358 \cdot 3$ & 762 & $71 \cdot 3$ & $132 \cdot 2$ & $106 \cdot 3$ & $126 \cdot 3$ & $270 \cdot 0$ & $(388 \cdot 8)$ \\
\hline Yoghurt and fermented milk & $16 \cdot 3$ & $47 \cdot 2$ & 0.0 & $104 \cdot 0$ & $215 \cdot 0$ & 189 & $17 \cdot 7$ & $92 \cdot 1$ & $75 \cdot 0$ & $83 \cdot 3$ & $250 \cdot 0$ & $(375 \cdot 0)$ \\
\hline Cheese and substitutes & $65 \cdot 6$ & $47 \cdot 0$ & $58 \cdot 5$ & $146 \cdot 4$ & $220 \cdot 8$ & 1043 & $97 \cdot 7$ & $67 \cdot 2$ & $46 \cdot 4$ & $60 \cdot 0$ & $147 \cdot 1$ & $220 \cdot 8$ \\
\hline Milk-based desserts and substitutes & $1 \cdot 2$ & 6.5 & 0.0 & $5 \cdot 1$ & $31 \cdot 3$ & 97 & $9 \cdot 1$ & 13.5 & $17 \cdot 4$ & $5 \cdot 1$ & $(50 \cdot 0)$ & $(125 \cdot 0)$ \\
\hline
\end{tabular}




\begin{tabular}{|c|c|c|c|c|c|c|c|c|c|c|c|c|}
\hline \multirow[b]{2}{*}{ Food categories } & \multicolumn{5}{|c|}{ Total population ( $n$ 1068) } & \multicolumn{7}{|c|}{ Consumers } \\
\hline & Mean & SD & Median & $95 \mathrm{th}^{*}$ & 99th* & $n$ & $\%$ & Mean & SD & Median & $95 t^{*}$ & 99 th* $^{*}$ \\
\hline Oils and fats & $45 \cdot 9$ & $18 \cdot 1$ & $43 \cdot 5$ & $76 \cdot 7$ & $101 \cdot 2$ & 1068 & $100 \cdot 0$ & $45 \cdot 9$ & $18 \cdot 1$ & $43 \cdot 5$ & $76 \cdot 7$ & $101 \cdot 2$ \\
\hline Olive oil & $36 \cdot 7$ & $14 \cdot 5$ & $35 \cdot 4$ & $61 \cdot 2$ & $79 \cdot \overline{5}$ & 1068 & $100 \cdot 0$ & $36 \cdot 7$ & $14 \cdot 5$ & $35 \cdot 4$ & $61 \cdot 2$ & $79 \cdot \overline{5}$ \\
\hline Other vegetable oils & $3 \cdot 1$ & $4 \cdot 6$ & 0.0 & $12 \cdot 1$ & $21 \cdot 1$ & 481 & $45 \cdot 0$ & $6 \cdot 9$ & $4 \cdot 6$ & $6 \cdot 1$ & $15 \cdot 4$ & $(27 \cdot 5)$ \\
\hline Butter and creams & $4 \cdot 8$ & $10 \cdot 0$ & 0.0 & $23 \cdot 0$ & $38 \cdot 5$ & 490 & $45 \cdot 9$ & $10 \cdot 5$ & $12 \cdot 5$ & $6 \cdot 7$ & $31 \cdot 2$ & $(50 \cdot 9)$ \\
\hline Other fats & $1 \cdot 3$ & $3 \cdot 6$ & 0.0 & $10 \cdot 0$ & $16 \cdot 7$ & 224 & $21 \cdot 0$ & $6 \cdot 2$ & $5 \cdot 5$ & $4 \cdot 7$ & $16 \cdot 4$ & $(22 \cdot 8)$ \\
\hline Eggs & $24 \cdot 4$ & $28 \cdot 2$ & $15 \cdot 6$ & $77 \cdot 0$ & $129 \cdot 2$ & 815 & $76 \cdot 3$ & $32 \cdot 0$ & $28 \cdot 2$ & 23.9 & $85 \cdot 0$ & 141.9 \\
\hline Alcoholic beverages and substitutes & $163 \cdot 1$ & $194 \cdot 9$ & $110 \cdot 1$ & $533 \cdot 6$ & $800 \cdot 0$ & 919 & $86 \cdot 0$ & $189 \cdot 6$ & $197 \cdot 8$ & $133 \cdot 3$ & $580 \cdot 6$ & $813 \cdot 3$ \\
\hline Regular wine and substitutes & $106 \cdot 5$ & $138 \cdot 5$ & $53 \cdot 3$ & 373.5 & $586 \cdot 7$ & 856 & $80 \cdot 1$ & $132 \cdot 9$ & $143 \cdot 0$ & $100 \cdot 0$ & $400 \cdot 2$ & $600 \cdot 0$ \\
\hline Beer, cider and substitutes & $52 \cdot 2$ & $119 \cdot 1$ & 0.0 & $266 \cdot 7$ & $533 \cdot 3$ & 319 & $29 \cdot 9$ & $174 \cdot 7$ & $161 \cdot 6$ & $133 \cdot 3$ & $466 \cdot 7$ & $(666 \cdot 7)$ \\
\hline $\begin{array}{l}\text { Sweet wine, spumante, wine-based appetizers, } \\
\text { spirits and liquors }\end{array}$ & 4.5 & $17 \cdot 2$ & 0.0 & 33.5 & $86 \cdot 7$ & 197 & $18 \cdot 4$ & $24 \cdot 4$ & $33 \cdot 7$ & $13 \cdot 3$ & $93 \cdot 3$ & $(173 \cdot 3)$ \\
\hline Sweet products and substitutes & $36 \cdot 3$ & $36 \cdot 8$ & $26 \cdot 7$ & $103 \cdot 5$ & $193 \cdot 7$ & 1025 & $96 \cdot 0$ & 37.9 & $36 \cdot 8$ & $28 \cdot 0$ & $104 \cdot 1$ & $193 \cdot 7$ \\
\hline Ice cream, ice lolly and substitutes & $10 \cdot 8$ & $29 \cdot 1$ & 0.0 & $66 \cdot 7$ & $166 \cdot 7$ & 220 & $20 \cdot 6$ & $52 \cdot 4$ & $44 \cdot 1$ & $33 \cdot 3$ & $164 \cdot 2$ & $(226 \cdot 7)$ \\
\hline Chocolate and substitutes & $2 \cdot 3$ & $6 \cdot 6$ & $0 \cdot 0$ & $15 \cdot 0$ & $33 \cdot 3$ & 230 & $21 \cdot 5$ & $10 \cdot 7$ & $10 \cdot 5$ & $6 \cdot 7$ & $33 \cdot 3$ & $(48 \cdot 1)$ \\
\hline Candies, jam and other sweet products (incl. sugar-free) & $3 \cdot 2$ & $10 \cdot 8$ & 0.0 & $17 \cdot 9$ & $40 \cdot 0$ & 274 & $25 \cdot 7$ & $12 \cdot 6$ & $18 \cdot 5$ & $7 \cdot 9$ & $36 \cdot 5$ & $(80 \cdot 0)$ \\
\hline Sugar, fructose, honey and other nutritious sweeteners & $19 \cdot \overline{4}$ & $16 \cdot 1$ & $16 \cdot 0$ & $48 \cdot 0$ & $72 \cdot 1$ & 965 & $90 \cdot 4$ & $21 \cdot 5$ & $15 \cdot 5$ & $18 \cdot 7$ & $49 \cdot 3$ & $72 \cdot 1$ \\
\hline Cocoa and cocoa-based powder & 0.5 & $2 \cdot 5$ & $0 \cdot 0$ & $3 \cdot 4$ & $14 \cdot 0$ & 94 & $8 \cdot 8$ & $5 \cdot 9$ & $6 \cdot 1$ & $4 \cdot 6$ & $(22 \cdot 3)$ & $(30 \cdot 0)$ \\
\hline Artificial sweeteners & 0.0 & 0.3 & 0.0 & $0 \cdot 0$ & $1 \cdot 3$ & 34 & $3 \cdot 2$ & $1 \cdot 2$ & $1 \cdot 4$ & $1 \cdot 0$ & $(3 \cdot 3)$ & $(7 \cdot 9)$ \\
\hline Meal substitutes & 0.1 & $1 \cdot 8$ & 0.0 & 0.0 & 0.0 & 3 & 0.3 & $31 \cdot 5$ & $18 \cdot 3$ & $31 \cdot 0$ & $(50 \cdot 0)$ & $(50 \cdot 0)$ \\
\hline Water and other non-alcoholic beverages & $851 \cdot 0$ & $475 \cdot 1$ & $786 \cdot 4$ & 1697.9 & $2426 \cdot 7$ & 1068 & $100 \cdot 0$ & $851 \cdot 0$ & $475 \cdot 1$ & $786 \cdot 4$ & 1697.9 & $2426 \cdot 7$ \\
\hline Tap water (as such, in beverages or recipes) & $175 \cdot 0$ & $291 \cdot 6$ & $11 \cdot 0$ & $810 \cdot 0$ & $1370 \cdot 0$ & 562 & $52 \cdot 6$ & $332 \cdot 6$ & $330 \cdot 6$ & $213 \cdot 3$ & $1013 \cdot 3$ & $(1493 \cdot 3)$ \\
\hline Bottled water & $474 \cdot 7$ & $429 \cdot 0$ & $426 \cdot 7$ & $1246 \cdot 7$ & $1813 \cdot 3$ & 831 & $77 \cdot 8$ & $610 \cdot 1$ & $392 \cdot 3$ & $560 \cdot 0$ & $1360 \cdot 0$ & $1880 \cdot 0$ \\
\hline Coffee, tea, herbal tea and substitutes & $135 \cdot 4$ & $108 \cdot 5$ & $111 \cdot 7$ & $330 \cdot 0$ & $500 \cdot 0$ & 1024 & $95 \cdot 9$ & $141 \cdot 2$ & $107 \cdot 0$ & $119 \cdot 6$ & $333 \cdot 3$ & $500 \cdot 0$ \\
\hline Fruit and vegetable juices & $30 \cdot 4$ & $75 \cdot 2$ & 1.5 & $200 \cdot 0$ & $330 \cdot 0$ & 601 & $56 \cdot 3$ & $54 \cdot 0$ & 93.8 & $6 \cdot 6$ & $204 \cdot 0$ & $(400 \cdot 0)$ \\
\hline Other soft drinks & $35 \cdot 5$ & $92 \cdot 4$ & 0.0 & $213 \cdot 3$ & $390 \cdot 0$ & 258 & $24 \cdot 2$ & $146 \cdot 8$ & $138 \cdot 1$ & $110 \cdot 0$ & $373 \cdot 3$ & $(810 \cdot 0)$ \\
\hline Miscellaneous & $3 \cdot 3$ & $4 \cdot 1$ & $2 \cdot 3$ & $10 \cdot 2$ & $19 \cdot 9$ & 827 & $77 \cdot 4$ & $4 \cdot 3$ & $4 \cdot 3$ & $2 \cdot 9$ & $11 \cdot 7$ & 23.9 \\
\hline Total amount of foods and beveragest & $2276 \cdot 2$ & $677 \cdot 9$ & $2189 \cdot 8$ & $3464 \cdot 2$ & $4125 \cdot 3$ & 1068 & $100 \cdot 0$ & $2276 \cdot 2$ & $677 \cdot 9$ & $2189 \cdot 8$ & $3464 \cdot 2$ & $4125 \cdot 3$ \\
\hline Total amount of liquid foods & $1111 \cdot 3$ & $492 \cdot 1$ & $1035 \cdot 3$ & $2057 \cdot 6$ & $2687 \cdot 6$ & 1068 & $100 \cdot 0$ & $1111 \cdot 3$ & $492 \cdot 1$ & $1035 \cdot 3$ & $2057 \cdot 6$ & $2687 \cdot 6$ \\
\hline Total amount of solid foods & $1164 \cdot 9$ & $327 \cdot 3$ & $1131 \cdot 5$ & $1765 \cdot 1$ & $2075 \cdot 2$ & 1068 & $100 \cdot 0$ & $1164 \cdot 9$ & $327 \cdot 3$ & $1131 \cdot 5$ & $1765 \cdot 1$ & $2075 \cdot 2$ \\
\hline
\end{tabular}

*High percentiles of consumption assessed on the basis of a $3 \mathrm{~d}$ survey provide an overestimate of long-term high levels of consumption. Values are enclosed in parentheses when the number of subjects to which they

refer is lower than 160 and 800 for the 95th and 99th percentile, respectively, since these values bear a large uncertainty and provide only a rough indication of high levels of consumption. 
Table 9 Mean, standard deviation, median and high percentiles of individual daily consumption ( $3 \mathrm{~d}$ average) by food category in the total population and in consumers ( $\mathrm{g} / \mathrm{d}$ ) - adults ( 18 to $64 \cdot 9$ years), females: Italian National Food Consumption Survey INRAN-SCAI 2005-06

\begin{tabular}{|c|c|c|c|c|c|c|c|c|c|c|c|c|}
\hline \multirow[b]{2}{*}{ Food categories } & \multicolumn{5}{|c|}{ Total population $(n 1245)$} & \multicolumn{7}{|c|}{ Consumers } \\
\hline & Mean & SD & Median & 95th* & 99th* & $n$ & $\%$ & Mean & SD & Median & $95 \mathrm{th}^{*}$ & 99th* \\
\hline Cereals, cereal products and substitutes & $232 \cdot 3$ & $90 \cdot 3$ & $228 \cdot 2$ & $383 \cdot 4$ & $481 \cdot 4$ & 1245 & $100 \cdot 0$ & $232 \cdot 3$ & $90 \cdot 3$ & $228 \cdot 2$ & $383 \cdot 4$ & $481 \cdot 4$ \\
\hline Bread & $86 \cdot 1$ & $63 \cdot 5$ & $75 \cdot 0$ & $210 \cdot 0$ & $260 \cdot 0$ & 1135 & $91 \cdot 2$ & $94 \cdot 4$ & $60 \cdot 3$ & $85 \cdot 0$ & $210 \cdot 0$ & $260 \cdot 0$ \\
\hline Pasta and pasta substitutes & $47 \cdot 7$ & $32 \cdot 1$ & $46 \cdot 7$ & $100 \cdot 0$ & $134 \cdot 8$ & 1104 & $88 \cdot 7$ & $53 \cdot 8$ & $28 \cdot 8$ & $52 \cdot 2$ & $102 \cdot 2$ & $137 \cdot 8$ \\
\hline Pizza & $7 \cdot 7$ & $23 \cdot 8$ & $0 \cdot 0$ & $66 \cdot 7$ & $112 \cdot 5$ & 177 & $14 \cdot 2$ & $54 \cdot 2$ & $38 \cdot 4$ & $40 \cdot 0$ & $116 \cdot 7$ & $(262 \cdot 5)$ \\
\hline Rice & $15 \cdot 1$ & $22 \cdot 6$ & $0 \cdot 0$ & $60 \cdot 5$ & $104 \cdot 2$ & 522 & $41 \cdot 9$ & $35 \cdot 9$ & $21 \cdot 6$ & $31 \cdot 6$ & $83 \cdot 3$ & $(106.9)$ \\
\hline Wheat, other cereals and flours & $36 \cdot 5$ & $34 \cdot 4$ & $29 \cdot 2$ & $97 \cdot 5$ & $129 \cdot 1$ & 1065 & $85 \cdot 5$ & $42 \cdot 7$ & $33 \cdot 5$ & $37 \cdot 0$ & $102 \cdot 0$ & $132 \cdot 7$ \\
\hline Breakfast cereals & $1 \cdot 7$ & $5 \cdot 9$ & 0.0 & $14 \cdot 0$ & $30 \cdot 0$ & 153 & $12 \cdot 3$ & $14 \cdot 2$ & $10 \cdot 5$ & $10 \cdot 0$ & $30 \cdot 3$ & $(49 \cdot 0)$ \\
\hline Biscuits & $12 \cdot 4$ & $18 \cdot 6$ & $4 \cdot 0$ & $47 \cdot 5$ & $93 \cdot 0$ & 646 & $51 \cdot 9$ & $23 \cdot 9$ & $19 \cdot 8$ & $18 \cdot 7$ & $60 \cdot 7$ & $(100 \cdot 0)$ \\
\hline Savoury fine bakery products & $9 \cdot 2$ & $16 \cdot 8$ & 0.0 & $38 \cdot 3$ & $59 \cdot 8$ & 569 & $45 \cdot 7$ & $20 \cdot 0$ & $20 \cdot 1$ & $16 \cdot 0$ & $48 \cdot 2$ & $(71 \cdot 6)$ \\
\hline Cakes and sweet snacks & $15 \cdot 9$ & $25 \cdot 9$ & $0 \cdot 0$ & $72 \cdot 9$ & $114 \cdot 6$ & 532 & $42 \cdot 7$ & $37 \cdot 2$ & $27 \cdot 8$ & $31 \cdot 3$ & $93 \cdot 1$ & $(133.8)$ \\
\hline Pulses, fresh and processed & $11 \cdot 2$ & $22 \cdot 8$ & 0.0 & $58 \cdot 7$ & $100 \cdot 1$ & 431 & $34 \cdot 6$ & $32 \cdot 4$ & $28 \cdot 7$ & $24 \cdot 9$ & $83 \cdot 3$ & $(136 \cdot 2)$ \\
\hline Vegetables, fresh and processed & $213 \cdot 1$ & $102 \cdot 7$ & $200 \cdot 2$ & 388.9 & $554 \cdot 4$ & 1244 & $99 \cdot 9$ & $213 \cdot 3$ & $102 \cdot 6$ & $200 \cdot 5$ & 388.9 & $554 \cdot 4$ \\
\hline Leafy vegetables, fresh & $45 \cdot 8$ & $51 \cdot 3$ & 31.9 & $143 \cdot 0$ & $233 \cdot 3$ & 1081 & $86 \cdot 8$ & $52 \cdot 7$ & $51 \cdot 7$ & $37 \cdot 2$ & $148 \cdot 7$ & $241 \cdot 5$ \\
\hline Tomatoes, fresh & $42 \cdot 1$ & $41 \cdot 5$ & $32 \cdot 8$ & $126 \cdot 7$ & $190 \cdot 0$ & 1054 & $84 \cdot 7$ & $49 \cdot 8$ & $40 \cdot 6$ & $39 \cdot 9$ & $132 \cdot 0$ & $195 \cdot 5$ \\
\hline Other fruiting vegetables, fresh & $32 \cdot 7$ & $51 \cdot 0$ & $8 \cdot 2$ & $135 \cdot 4$ & $205 \cdot 1$ & 846 & $68 \cdot 0$ & $48 \cdot 1$ & $55 \cdot 5$ & $30 \cdot 0$ & $154 \cdot 0$ & $240 \cdot 0$ \\
\hline Roots and onions, fresh & $19 \cdot 1$ & $25 \cdot 4$ & $9 \cdot \overline{6}$ & $71 \cdot 8$ & $114 \cdot 4$ & 1210 & $97 \cdot 2$ & $19 \cdot 7$ & $25 \cdot 6$ & $10 \cdot 2$ & $74 \cdot 7$ & $114 \cdot 4$ \\
\hline Other vegetables, fresh & $39 \cdot 1$ & $46 \cdot 7$ & $22 \cdot 6$ & $135 \cdot 6$ & $211 \cdot 6$ & 1052 & $84 \cdot 5$ & $46 \cdot 2$ & $47 \cdot 4$ & $31 \cdot 9$ & $142 \cdot 5$ & $215 \cdot 8$ \\
\hline Vegetables, processed & $32 \cdot 4$ & $30 \cdot 6$ & $31 \cdot 5$ & $85 \cdot 8$ & $128 \cdot 9$ & 933 & $74 \cdot 9$ & $43 \cdot 3$ & $27 \cdot 9$ & $34 \cdot 5$ & $94 \cdot 1$ & $134 \cdot 0$ \\
\hline Spices and herbs & $1 \cdot 9$ & $2 \cdot 9$ & $1 \cdot 0$ & $5 \cdot 9$ & $14 \cdot 0$ & 1050 & $84 \cdot 3$ & $2 \cdot 2$ & $3 \cdot 1$ & $1 \cdot 4$ & $6 \cdot 3$ & $15 \cdot 4$ \\
\hline Potatoes, tubers and their products & $46 \cdot 6$ & $53 \cdot 2$ & $34 \cdot 2$ & $145 \cdot 8$ & $200 \cdot 0$ & 833 & $66 \cdot 9$ & $69 \cdot 6$ & $51 \cdot 3$ & $62 \cdot 5$ & $163 \cdot 4$ & $219 \cdot 9$ \\
\hline Fruit, fresh and processed & $216 \cdot 5$ & $148 \cdot 8$ & $198 \cdot 6$ & 493.5 & $666 \cdot 0$ & 1181 & 94.9 & $228 \cdot 2$ & $143 \cdot 8$ & $206 \cdot 0$ & $499 \cdot 7$ & $681 \cdot 6$ \\
\hline Citrus fruit, fresh & $49 \cdot 4$ & $86 \cdot 9$ & $2 \cdot 0$ & $222 \cdot 7$ & $381 \cdot 6$ & 636 & $51 \cdot 1$ & $96 \cdot 8$ & $101 \cdot 0$ & $60 \cdot 7$ & $303 \cdot 3$ & $(440 \cdot 0)$ \\
\hline Exotic fruit, fresh & $16 \cdot 8$ & $27 \cdot 8$ & $0 \cdot 0$ & $66 \cdot 7$ & $125 \cdot 0$ & 493 & $39 \cdot 6$ & $42 \cdot 3$ & $29 \cdot 5$ & $33 \cdot 3$ & $100 \cdot 0$ & $(150 \cdot 0)$ \\
\hline Other fruit, fresh & $147 \cdot 6$ & $128 \cdot 9$ & $126 \cdot 3$ & $401 \cdot 7$ & $555 \cdot 3$ & 1069 & $85 \cdot 9$ & $171 \cdot 9$ & $123 \cdot 2$ & $150 \cdot 0$ & $418 \cdot 0$ & $564 \cdot 0$ \\
\hline Nuts, seeds, olives and their products, dried fruit & $2 \cdot 5$ & $6 \cdot 3$ & $0 \cdot 0$ & $13 \cdot 3$ & $30 \cdot 8$ & 333 & $26 \cdot 7$ & $9 \cdot 4$ & $9 \cdot 1$ & $7 \cdot 4$ & $26 \cdot 0$ & $(51 \cdot 7)$ \\
\hline Other processed fruit (in syrup, in purée, etc.) & $0 \cdot 2$ & $2 \cdot 4$ & $0 \cdot 0$ & 0.0 & 0.6 & 16 & $1 \cdot 3$ & $13 \cdot 1$ & $17 \cdot 8$ & $1 \cdot 3$ & $(50 \cdot 0)$ & $(50 \cdot 0)$ \\
\hline Meat, meat products and substitutes & $97 \cdot 3$ & $52 \cdot 8$ & $91 \cdot 4$ & $190 \cdot 1$ & $252 \cdot 0$ & 1231 & 98.9 & $98 \cdot 4$ & $52 \cdot 1$ & $92 \cdot 6$ & $190 \cdot 8$ & $252 \cdot 0$ \\
\hline Beef and veal, not preserved, excl. offal & $38 \cdot 0$ & $37 \cdot 6$ & $33 \cdot 3$ & $108 \cdot 8$ & $156 \cdot 6$ & 908 & $72 \cdot 9$ & $52 \cdot 1$ & $34 \cdot 7$ & $41 \cdot 5$ & $117 \cdot 7$ & $179 \cdot 3$ \\
\hline Pork, not preserved, excl. offal & $11 \cdot 6$ & $23 \cdot 6$ & $0 \cdot 0$ & $58 \cdot 3$ & $96 \cdot 9$ & 368 & $29 \cdot 6$ & $39 \cdot 1$ & $28 \cdot 5$ & $37 \cdot 6$ & $93 \cdot 6$ & $(140 \cdot 2)$ \\
\hline Poultry and game, not preserved, excl. offal & $18 \cdot 5$ & $27 \cdot 3$ & $0 \cdot 0$ & $72 \cdot 6$ & $103 \cdot 7$ & 492 & $39 \cdot 5$ & $46 \cdot 8$ & $23 \cdot 6$ & $39 \cdot 5$ & $98 \cdot 9$ & $(122 \cdot 1)$ \\
\hline Other meats, not preserved, excl. offal & $4 \cdot 8$ & $16 \cdot 6$ & $0 \cdot 0$ & $41 \cdot 7$ & $82 \cdot 3$ & 119 & $9 \cdot 6$ & $50 \cdot 7$ & $24 \cdot 1$ & $41 \cdot 8$ & $(101 \cdot 4)$ & $(127 \cdot 1)$ \\
\hline $\begin{array}{l}\text { Ham, salami, sausages and other preserved meats, } \\
\text { excl. offal }\end{array}$ & $23 \cdot 3$ & $23 \cdot 2$ & $20 \cdot 0$ & $66 \cdot 3$ & $98 \cdot 2$ & 991 & $79 \cdot 6$ & $29 \cdot 3$ & $22 \cdot 4$ & $23 \cdot 7$ & $72 \cdot 0$ & $107 \cdot 8$ \\
\hline Offal, blood and their products & $1 \cdot 0$ & $7 \cdot 5$ & $0 \cdot 0$ & 0.0 & $37 \cdot 3$ & 35 & $2 \cdot 8$ & $36 \cdot 2$ & $27 \cdot 0$ & $33 \cdot 3$ & $(82 \cdot 3)$ & $(128 \cdot 7)$ \\
\hline Meat substitutes & 0.1 & $2 \cdot 1$ & 0.0 & 0.0 & 0.0 & 4 & $0 \cdot 3$ & $35 \cdot 4$ & $10 \cdot 5$ & $33 \cdot 3$ & $(50 \cdot 0)$ & $(50 \cdot 0)$ \\
\hline Fish, seafood and their products & $44 \cdot 7$ & $49 \cdot 2$ & $36 \cdot 5$ & $142 \cdot 6$ & $199 \cdot 0$ & 852 & $68 \cdot 4$ & $65 \cdot 4$ & $46 \cdot 7$ & 57.5 & $157 \cdot 4$ & $214 \cdot 4$ \\
\hline Fish and seafood, fresh and frozen & $38 \cdot 7$ & $47 \cdot 8$ & $15 \cdot 2$ & $137 \cdot 2$ & $184 \cdot 6$ & 680 & $54 \cdot 6$ & $70 \cdot 9$ & $43 \cdot 6$ & $57 \cdot 5$ & $158 \cdot 4$ & $(209 \cdot 0)$ \\
\hline Fish and seafood, preserved & $6 \cdot 0$ & $12 \cdot 4$ & $0 \cdot 0$ & $31 \cdot 9$ & $58 \cdot 4$ & 414 & $33 \cdot 3$ & $18 \cdot 2$ & $15 \cdot 4$ & $13 \cdot 5$ & $50 \cdot 0$ & $(67 \cdot 1)$ \\
\hline Milk, milk products and substitutes & 192.9 & $117 \cdot 8$ & $183 \cdot 3$ & $405 \cdot 0$ & $504 \cdot 5$ & 1235 & $99 \cdot 2$ & $194 \cdot 5$ & $117 \cdot 0$ & $184 \cdot 5$ & $407 \cdot 5$ & $504 \cdot 5$ \\
\hline $\begin{array}{l}\text { Milk, milk-based beverages, human milk, infant formula } \\
\text { and substitutes }\end{array}$ & $110 \cdot 5$ & $96 \cdot 4$ & $112 \cdot 5$ & $260 \cdot 9$ & $350 \cdot 0$ & 993 & $79 \cdot 8$ & $138 \cdot 5$ & $88 \cdot 1$ & $135 \cdot 0$ & $277 \cdot 7$ & $357 \cdot 1$ \\
\hline Yoghurt and fermented milk & $26 \cdot 8$ & $52 \cdot 4$ & 0.0 & $125 \cdot 0$ & $211 \cdot 7$ & 386 & $31 \cdot 0$ & $86 \cdot 6$ & $60 \cdot 8$ & $83 \cdot 3$ & $200 \cdot 0$ & $(346 \cdot 7)$ \\
\hline Cheese and substitutes & $54 \cdot 5$ & $39 \cdot 0$ & $50 \cdot 0$ & $123 \cdot 9$ & $167 \cdot 5$ & 1202 & $96 \cdot 5$ & $56 \cdot 4$ & $38 \cdot 3$ & $51 \cdot 7$ & $124 \cdot 3$ & $167 \cdot 5$ \\
\hline Milk-based desserts and substitutes & $1 \cdot 1$ & $8 \cdot 7$ & $0 \cdot 0$ & $5 \cdot 1$ & $33 \cdot 3$ & 75 & $6 \cdot 0$ & $18 \cdot 5$ & $30 \cdot 7$ & $5 \cdot 1$ & $(60 \cdot 0)$ & $(175 \cdot 0)$ \\
\hline
\end{tabular}




\begin{tabular}{|c|c|c|c|c|c|c|c|c|c|c|c|c|}
\hline \multirow[b]{2}{*}{ Food categories } & \multicolumn{5}{|c|}{ Total population ( $n 1245$ ) } & \multicolumn{7}{|c|}{ Consumers } \\
\hline & Mean & SD & Median & 95th* & 99th* & $n$ & $\%$ & Mean & SD & Median & 95th* & 99th* \\
\hline Oils and fats & $38 \cdot 8$ & $14 \cdot 3$ & $37 \cdot 7$ & $64 \cdot 0$ & $78 \cdot 0$ & 1245 & $100 \cdot 0$ & $38 \cdot 8$ & $14 \cdot 3$ & $37 \cdot 7$ & $64 \cdot 0$ & $78 \cdot 0$ \\
\hline Olive oil & $31 \cdot 4$ & $12 \cdot 4$ & $30 \cdot 2$ & $52 \cdot 6$ & $63 \cdot 2$ & 1245 & $100 \cdot 0$ & $31 \cdot 4$ & $12 \cdot 4$ & $30 \cdot 2$ & $52 \cdot 6$ & $63 \cdot 2$ \\
\hline Other vegetable oils & $2 \cdot 6$ & $3 \cdot 7$ & 0.0 & $9 \cdot 4$ & $14 \cdot 5$ & 553 & $44 \cdot 4$ & $5 \cdot 9$ & 3.5 & $6 \cdot 1$ & $12 \cdot 0$ & $(16 \cdot 8)$ \\
\hline Butter and creams & $3 \cdot 9$ & $7 \cdot 0$ & $0 \cdot 0$ & $16 \cdot 4$ & $31 \cdot 5$ & 569 & $45 \cdot 7$ & 8.5 & $8 \cdot 1$ & $6 \cdot 1$ & 23.9 & $(42 \cdot 2)$ \\
\hline Other fats & 0.8 & $2 \cdot 6$ & 0.0 & $5 \cdot 3$ & $12 \cdot 2$ & 219 & $17 \cdot 6$ & $4 \cdot 8$ & $4 \cdot 3$ & $3 \cdot 4$ & $14 \cdot 0$ & $(16 \cdot 8)$ \\
\hline Eggs & $18 \cdot 7$ & $21 \cdot 5$ & $11 \cdot 5$ & $61 \cdot 0$ & $89 \cdot 9$ & 906 & $72 \cdot 8$ & $25 \cdot 7$ & $21 \cdot 3$ & $19 \cdot 9$ & $67 \cdot 5$ & 94.0 \\
\hline Alcoholic beverages and substitutes & $58 \cdot 2$ & $95 \cdot 0$ & 0.1 & $253 \cdot 3$ & $406 \cdot 7$ & 895 & $71 \cdot 9$ & $80 \cdot 9$ & $103 \cdot 6$ & $40 \cdot 1$ & $293 \cdot 3$ & $480 \cdot 0$ \\
\hline Regular wine and substitutes & $38 \cdot 4$ & $68 \cdot 7$ & 0.0 & $200 \cdot 0$ & $266 \cdot 7$ & 820 & $65 \cdot 9$ & $58 \cdot 3$ & $77 \cdot 4$ & $20 \cdot 1$ & $220 \cdot 0$ & $293 \cdot 3$ \\
\hline Beer, cider and substitutes & $17 \cdot 9$ & $55 \cdot 2$ & $0 \cdot 0$ & $133 \cdot 3$ & $266 \cdot 7$ & 200 & $16 \cdot 1$ & $111 \cdot 6$ & $92 \cdot 3$ & $80 \cdot 0$ & $276 \cdot 7$ & $(441 \cdot 7)$ \\
\hline $\begin{array}{l}\text { Sweet wine, spumante, wine-based appetizers, } \\
\text { spirits and liquors }\end{array}$ & $1 \cdot 8$ & $8 \cdot 5$ & 0.0 & $13 \cdot 3$ & $40 \cdot 1$ & 156 & $12 \cdot 5$ & $14 \cdot 5$ & $20 \cdot 0$ & $1 \cdot 5$ & $(53 \cdot 3)$ & $(80 \cdot 0)$ \\
\hline Sweet products and substitutes & $31 \cdot 2$ & $29 \cdot 9$ & $22 \cdot 8$ & $87 \cdot 7$ & $143 \cdot 8$ & 1166 & $93 \cdot 7$ & $33 \cdot 3$ & $29 \cdot 7$ & 24.5 & 89.9 & $144 \cdot 4$ \\
\hline Ice cream, ice lolly and substitutes & $8 \cdot 8$ & $22 \cdot 8$ & $0 \cdot 0$ & $58 \cdot 3$ & $113 \cdot 3$ & 253 & $20 \cdot 3$ & $43 \cdot 4$ & $32 \cdot 4$ & $33 \cdot 3$ & $113 \cdot 3$ & $(166 \cdot 7)$ \\
\hline Chocolate and substitutes & $2 \cdot 0$ & $6 \cdot 0$ & $0 \cdot 0$ & $11 \cdot 7$ & $30 \cdot 7$ & 253 & $20 \cdot 3$ & $9 \cdot 8$ & $10 \cdot 1$ & $6 \cdot 7$ & $30 \cdot 7$ & $(51 \cdot 1)$ \\
\hline Candies, jam and other sweet products (incl. sugar-free) & $3 \cdot 6$ & $8 \cdot 0$ & $0 \cdot 0$ & $20 \cdot 0$ & $37 \cdot 3$ & 366 & $29 \cdot 4$ & $12 \cdot 1$ & $10 \cdot 8$ & $9 \cdot 9$ & $31 \cdot 6$ & $(47 \cdot 7)$ \\
\hline Sugar, fructose, honey and other nutritious sweeteners & $16 \cdot 3$ & $13 \cdot 9$ & $13 \cdot 9$ & $42 \cdot 7$ & $61 \cdot 2$ & 1074 & $86 \cdot 3$ & $18 \cdot 9$ & $13 \cdot 2$ & $16 \cdot 0$ & $45 \cdot 3$ & $61 \cdot 3$ \\
\hline Cocoa and cocoa-based powder & 0.5 & $2 \cdot 4$ & 0.0 & $2 \cdot 6$ & $12 \cdot 1$ & 101 & $8 \cdot 1$ & $5 \cdot 9$ & $6 \cdot 2$ & $4 \cdot 6$ & $(17 \cdot 0)$ & $(21 \cdot 7)$ \\
\hline Artificial sweeteners & $0 \cdot 1$ & 0.4 & 0.0 & $0 \cdot 1$ & $2 \cdot 3$ & 72 & $5 \cdot 8$ & $1 \cdot 2$ & $1 \cdot 1$ & 0.8 & $(4 \cdot 2)$ & $(4 \cdot 7)$ \\
\hline Meal substitutes & 0.0 & 0.7 & 0.0 & 0.0 & 0.0 & 1 & $0 \cdot 1$ & $26 \cdot 0$ & - & $26 \cdot 0$ & $(26 \cdot 0)$ & $(26 \cdot 0)$ \\
\hline Water and other non-alcoholic beverages & $882 \cdot 6$ & $423 \cdot 9$ & $812 \cdot 2$ & $1680 \cdot 0$ & $2195 \cdot 8$ & 1245 & $100 \cdot 0$ & $882 \cdot 6$ & $423 \cdot 9$ & $812 \cdot 2$ & $1680 \cdot 0$ & $2195 \cdot 8$ \\
\hline Tap water (as such, in beverages or recipes) & $192 \cdot 9$ & $308 \cdot 3$ & $53 \cdot 3$ & $893 \cdot 3$ & $1333 \cdot 3$ & 678 & $54 \cdot 5$ & $354 \cdot 2$ & $342 \cdot 6$ & $250 \cdot 0$ & $1053 \cdot 3$ & $(1485 \cdot 4)$ \\
\hline Bottled water & $498 \cdot 1$ & $429 \cdot 4$ & $480 \cdot 0$ & $1246 \cdot 7$ & $1900 \cdot 0$ & 984 & $79 \cdot 0$ & $630 \cdot 2$ & $387 \cdot 3$ & $586 \cdot 7$ & $1346 \cdot 7$ & $1946 \cdot 7$ \\
\hline Coffee, tea, herbal tea and substitutes & $138 \cdot 3$ & $113 \cdot 3$ & $115 \cdot 8$ & $355 \cdot 0$ & $536 \cdot 7$ & 1188 & $95 \cdot 4$ & 144.9 & $111 \cdot 7$ & $120 \cdot 0$ & $360 \cdot 8$ & $540 \cdot 0$ \\
\hline Fruit and vegetable juices & $29 \cdot 7$ & $68 \cdot 0$ & 1.5 & $170 \cdot 0$ & $309 \cdot 7$ & 698 & $56 \cdot 1$ & $52 \cdot 9$ & $83 \cdot 8$ & $6 \cdot 8$ & $203 \cdot 5$ & $(334 \cdot 8)$ \\
\hline Other soft drinks & $23 \cdot 7$ & $61 \cdot 6$ & 0.0 & $141 \cdot 7$ & $266 \cdot 7$ & 259 & $20 \cdot 8$ & 113.9 & $89 \cdot 5$ & $106 \cdot 7$ & $266 \cdot 7$ & $(480 \cdot 0)$ \\
\hline Miscellaneous & $3 \cdot 3$ & 3.9 & $2 \cdot 4$ & $10 \cdot 6$ & $17 \cdot 3$ & 982 & $78 \cdot 9$ & $4 \cdot 2$ & 3.9 & 2.9 & $11 \cdot 2$ & $20 \cdot 4$ \\
\hline Total amount of foods and beveragest & $2087 \cdot 2$ & $587 \cdot 1$ & $2039 \cdot 3$ & $3133 \cdot 8$ & $3607 \cdot 7$ & 1245 & $100 \cdot 0$ & $2087 \cdot 2$ & $587 \cdot 1$ & $2039 \cdot 3$ & $3133 \cdot 8$ & $3607 \cdot 7$ \\
\hline Total amount of liquid foods & $1053 \cdot 8$ & $430 \cdot 5$ & $990 \cdot 8$ & $1912 \cdot 0$ & $2375 \cdot 3$ & 1245 & $100 \cdot 0$ & $1053 \cdot 8$ & $430 \cdot 5$ & $990 \cdot 8$ & $1912 \cdot 0$ & $2375 \cdot 3$ \\
\hline Total amount of solid foods & $1033 \cdot 9$ & $278 \cdot 7$ & $1029 \cdot 1$ & $1459 \cdot 3$ & $1846 \cdot 2$ & 1245 & $100 \cdot 0$ & $1033 \cdot 9$ & $278 \cdot 7$ & $1029 \cdot 1$ & $1459 \cdot 3$ & $1846 \cdot 2$ \\
\hline
\end{tabular}

*High percentiles of consumption assessed on the basis of a $3 \mathrm{~d}$ survey provide an overestimate of long-term high levels of consumption. Values are enclosed in parentheses when the number of subjects to which they

refer is lower than 160 and 800 for the 95th and 99th percentile, respectively, since these values bear a large uncertainty and provide only a rough indication of high levels of consumption. 
Table 10 Mean, standard deviation, median and high percentiles of individual daily consumption ( $3 \mathrm{~d}$ average) by food category in the total population and in consumers ( $\mathrm{g} / \mathrm{d})$ - elderly ( $\geq 65$ years), males: Italian National Food Consumption Survey INRAN-SCAI 2005-06

\begin{tabular}{|c|c|c|c|c|c|c|c|c|c|c|c|c|}
\hline \multirow[b]{2}{*}{ Food categories } & \multicolumn{5}{|c|}{ Total population $(n$ 202) } & \multicolumn{7}{|c|}{ Consumers } \\
\hline & Mean & SD & Median & $95 \mathrm{th}^{*}$ & 99 th $^{*}$ & $n$ & $\%$ & Mean & SD & Median & 95th* & 99th* \\
\hline Cereals, cereal products and substitutes & $283 \cdot 2$ & $101 \cdot 7$ & $269 \cdot 9$ & $466 \cdot 0$ & $(597 \cdot 4)$ & 202 & $100 \cdot 0$ & $283 \cdot 2$ & $101 \cdot 7$ & $269 \cdot 9$ & $466 \cdot 0$ & $(597 \cdot 4)$ \\
\hline Bread & $137 \cdot 6$ & $90 \cdot 8$ & $120 \cdot 0$ & 298.9 & $(447 \cdot 6)$ & 195 & $96 \cdot 5$ & $142 \cdot 5$ & 88.5 & $120 \cdot 0$ & $300 \cdot 0$ & $(455 \cdot 0)$ \\
\hline Pasta and pasta substitutes & $61 \cdot 1$ & $31 \cdot 1$ & $61 \cdot 2$ & $109 \cdot 6$ & $(129 \cdot 8)$ & 192 & $95 \cdot 0$ & $64 \cdot 3$ & $28 \cdot 5$ & $62 \cdot 7$ & $116 \cdot 5$ & $(131 \cdot 2)$ \\
\hline Pizza & $4 \cdot 6$ & $17 \cdot 5$ & 0.0 & $33 \cdot 3$ & $(112 \cdot 5)$ & 18 & 8.9 & $51 \cdot 9$ & 31.9 & $39 \cdot 6$ & $(116 \cdot 7)$ & $(116 \cdot 7)$ \\
\hline Rice & $17 \cdot 2$ & $25 \cdot 1$ & $0 \cdot 0$ & $67 \cdot 8$ & $(97 \cdot 1)$ & 90 & $44 \cdot 6$ & $38 \cdot 7$ & $24 \cdot 2$ & 33.9 & $(86 \cdot 8)$ & $(156 \cdot 6)$ \\
\hline Wheat, other cereals and flours & $27 \cdot 2$ & $30 \cdot 4$ & $16 \cdot 9$ & $79 \cdot 3$ & $(109 \cdot 9)$ & 158 & $78 \cdot 2$ & $34 \cdot 8$ & $30 \cdot 4$ & $28 \cdot 0$ & $(87 \cdot 7)$ & $(167 \cdot 7)$ \\
\hline Breakfast cereals & 0.5 & $3 \cdot 3$ & 0.0 & $0 \cdot 0$ & $(21 \cdot 0)$ & 6 & $3 \cdot 0$ & $17 \cdot 6$ & $8 \cdot 3$ & $17 \cdot 5$ & $(30 \cdot 0)$ & $(30 \cdot 0)$ \\
\hline Biscuits & $10 \cdot 4$ & $18 \cdot 9$ & $0 \cdot 0$ & $50 \cdot 7$ & $(73 \cdot 7)$ & 84 & $41 \cdot 6$ & $24 \cdot 9$ & $22 \cdot 3$ & $18 \cdot 7$ & $(63 \cdot 3)$ & $(133 \cdot 3)$ \\
\hline Savoury fine bakery products & $10 \cdot 3$ & $18 \cdot 7$ & $0 \cdot 0$ & $40 \cdot 0$ & $(100 \cdot 0)$ & 81 & $40 \cdot 1$ & $25 \cdot 6$ & $21 \cdot 8$ & $16 \cdot 7$ & $(72 \cdot 0)$ & $(108 \cdot 3)$ \\
\hline Cakes and sweet snacks & $14 \cdot 3$ & $28 \cdot 9$ & $0 \cdot 0$ & $62 \cdot 5$ & $(114 \cdot 6)$ & 56 & $27 \cdot 7$ & $51 \cdot 6$ & $33 \cdot 0$ & $41 \cdot 7$ & $(114 \cdot 6)$ & $(208 \cdot 3)$ \\
\hline Pulses, fresh and processed & $14 \cdot 1$ & $27 \cdot 5$ & 0.0 & $59 \cdot 7$ & (133.7) & 80 & $39 \cdot 6$ & $35 \cdot 6$ & 33.9 & $23 \cdot 2$ & $(79 \cdot 7)$ & $(205 \cdot 0)$ \\
\hline Vegetables, fresh and processed & $243 \cdot 5$ & $120 \cdot 8$ & $221 \cdot 9$ & $440 \cdot 8$ & $(605 \cdot 2)$ & 202 & $100 \cdot 0$ & 243.5 & $120 \cdot 8$ & $221 \cdot 9$ & $440 \cdot 8$ & $(605 \cdot 2)$ \\
\hline Leafy vegetables, fresh & $57 \cdot 2$ & $68 \cdot 8$ & $38 \cdot 7$ & $178 \cdot 6$ & $(243 \cdot 3)$ & 184 & $91 \cdot 1$ & $62 \cdot 8$ & $69 \cdot 6$ & $41 \cdot 7$ & $181 \cdot 0$ & $(261 \cdot 6)$ \\
\hline Tomatoes, fresh & $41 \cdot 5$ & $44 \cdot 4$ & $30 \cdot 3$ & $122 \cdot 3$ & $(167 \cdot 6)$ & 164 & $81 \cdot 2$ & $51 \cdot 1$ & 43.9 & $39 \cdot 5$ & $133 \cdot 3$ & $(253 \cdot 1)$ \\
\hline Other fruiting vegetables, fresh & $32 \cdot 0$ & $52 \cdot 0$ & $7 \cdot 5$ & $153 \cdot 7$ & $(194 \cdot 0)$ & 145 & $71 \cdot 8$ & $44 \cdot 6$ & $56 \cdot 7$ & $20 \cdot 0$ & $(167 \cdot 4)$ & $(206 \cdot 0)$ \\
\hline Roots and onions, fresh & $23 \cdot 9$ & $24 \cdot 2$ & $15 \cdot 1$ & $77 \cdot 6$ & $(99 \cdot 8)$ & 201 & $99 \cdot 5$ & $24 \cdot 1$ & $24 \cdot 2$ & $15 \cdot 3$ & $77 \cdot 6$ & $(99 \cdot 8)$ \\
\hline Other vegetables, fresh & $45 \cdot 0$ & $49 \cdot 3$ & $28 \cdot 9$ & $148 \cdot 8$ & $(185 \cdot 8)$ & 175 & $86 \cdot 6$ & $51 \cdot 9$ & $49 \cdot 4$ & $42 \cdot 6$ & $153 \cdot 2$ & $(231 \cdot 1)$ \\
\hline Vegetables, processed & $41 \cdot 7$ & $36 \cdot 9$ & $37 \cdot 8$ & $110 \cdot 7$ & $(154 \cdot 6)$ & 166 & $82 \cdot 2$ & $50 \cdot 8$ & $34 \cdot 6$ & $41 \cdot 7$ & $118 \cdot 1$ & $(191 \cdot 7)$ \\
\hline Spices and herbs & $2 \cdot 2$ & $3 \cdot 2$ & $1 \cdot 2$ & $6 \cdot 7$ & $(12 \cdot 8)$ & 171 & $84 \cdot 7$ & $2 \cdot 5$ & $3 \cdot 4$ & $1 \cdot 6$ & $7 \cdot 4$ & $(23.9)$ \\
\hline Potatoes, tubers and their products & $61 \cdot 3$ & $57 \cdot 1$ & $56 \cdot 6$ & $165 \cdot 1$ & $(222 \cdot 3)$ & 155 & $76 \cdot 7$ & $79 \cdot 9$ & $52 \cdot 5$ & $65 \cdot 4$ & $(166 \cdot 1)$ & $(232 \cdot 8)$ \\
\hline Fruit, fresh and processed & $260 \cdot 3$ & $193 \cdot 9$ & $241 \cdot 1$ & $519 \cdot 3$ & $(996 \cdot 7)$ & 195 & 96.5 & $269 \cdot 7$ & $190 \cdot 8$ & $244 \cdot 7$ & $522 \cdot 7$ & (1384.0) \\
\hline Citrus fruit, fresh & $52 \cdot 0$ & $84 \cdot 4$ & $2 \cdot 0$ & $242 \cdot 7$ & $(368 \cdot 0)$ & 107 & $53 \cdot 0$ & $98 \cdot 2$ & $94 \cdot 6$ & $60 \cdot 7$ & $(303 \cdot 3)$ & $(384 \cdot 7)$ \\
\hline Exotic fruit, fresh & $14 \cdot 3$ & $27 \cdot 0$ & $0 \cdot 0$ & $66 \cdot 7$ & $(117 \cdot 9)$ & 69 & $34 \cdot 2$ & $41 \cdot 9$ & $31 \cdot 3$ & $33 \cdot 3$ & $(100 \cdot 0)$ & $(166 \cdot 7)$ \\
\hline Other fruit, fresh & $189 \cdot 3$ & $181 \cdot 0$ & $150 \cdot 0$ & $460 \cdot 0$ & $(908 \cdot 0)$ & 180 & $89 \cdot 1$ & $212 \cdot 4$ & $178 \cdot 4$ & $168 \cdot 8$ & $482 \cdot 2$ & $(996 \cdot 7)$ \\
\hline Nuts, seeds, olives and their products, dried fruit & $3 \cdot 7$ & $10 \cdot 6$ & $0 \cdot 0$ & $19 \cdot 7$ & $(42 \cdot 3)$ & 58 & $28 \cdot 7$ & $12 \cdot 8$ & $16 \cdot 6$ & $7 \cdot 4$ & $(42 \cdot 3)$ & $(112 \cdot 0)$ \\
\hline Other processed fruit (in syrup, in purée, etc.) & $1 \cdot 0$ & $9 \cdot 8$ & $0 \cdot 0$ & 0.0 & $(25 \cdot 0)$ & 5 & $2 \cdot 5$ & $41 \cdot 4$ & $52 \cdot 3$ & $25 \cdot 0$ & $(126 \cdot 7)$ & $(126 \cdot 7)$ \\
\hline Meat, meat products and substitutes & $117 \cdot 8$ & $62 \cdot 3$ & $113 \cdot 9$ & $225 \cdot 2$ & $(265 \cdot 8)$ & 202 & $100 \cdot 0$ & $117 \cdot 8$ & $62 \cdot 3$ & $113 \cdot 9$ & $225 \cdot 2$ & $(265 \cdot 8)$ \\
\hline Beef and veal, not preserved, excl. offal & $51 \cdot 9$ & $44 \cdot 5$ & $43 \cdot 0$ & $136 \cdot 0$ & $(169 \cdot 6)$ & 157 & $77 \cdot 7$ & $66 \cdot 8$ & $39 \cdot 3$ & $62 \cdot 2$ & $(145 \cdot 2)$ & $(184 \cdot 8)$ \\
\hline Pork, not preserved, excl. offal & $11 \cdot 5$ & $24 \cdot 5$ & 0.0 & $46 \cdot 9$ & $(121 \cdot 7)$ & 71 & $35 \cdot 1$ & $32 \cdot 6$ & $32 \cdot 0$ & $25 \cdot 0$ & $(82 \cdot 1)$ & $(195 \cdot 9)$ \\
\hline Poultry and game, not preserved, excl. offal & $20 \cdot 5$ & $30 \cdot 7$ & $0 \cdot 0$ & $83 \cdot 3$ & $(114 \cdot 2)$ & 79 & $39 \cdot 1$ & $52 \cdot 4$ & $27 \cdot 1$ & $48 \cdot 8$ & $(111 \cdot 2)$ & $(168 \cdot 7)$ \\
\hline Other meats, not preserved, excl. offal & $7 \cdot 2$ & $24 \cdot 0$ & $0 \cdot 0$ & $52 \cdot 0$ & $(131 \cdot 6)$ & 22 & $10 \cdot 9$ & $66 \cdot 3$ & $37 \cdot 4$ & $51 \cdot 3$ & $(131 \cdot 6)$ & $(134 \cdot 3)$ \\
\hline $\begin{array}{l}\text { Ham, salami, sausages and other preserved meats, } \\
\text { excl. offal }\end{array}$ & $24 \cdot 7$ & $24 \cdot 3$ & $20 \cdot 0$ & $71 \cdot 2$ & $(104 \cdot 2)$ & 162 & $80 \cdot 2$ & $30 \cdot 7$ & $23 \cdot 5$ & $26 \cdot 5$ & $73 \cdot 7$ & $(107 \cdot 9)$ \\
\hline Offal, blood and their products & $1 \cdot 9$ & $9 \cdot 9$ & $0 \cdot 0$ & $0 \cdot 0$ & $(57 \cdot 5)$ & 10 & $5 \cdot 0$ & $38 \cdot 6$ & $24 \cdot 7$ & $35 \cdot 3$ & $(83 \cdot 3)$ & $(83 \cdot 3)$ \\
\hline Meat substitutes & $0 \cdot 1$ & $1 \cdot 8$ & $0 \cdot 0$ & $0 \cdot 0$ & $(0 \cdot 0)$ & 1 & 0.5 & $25 \cdot 0$ & - & $25 \cdot 0$ & $(25 \cdot 0)$ & $(25 \cdot 0)$ \\
\hline Fish, seafood and their products & $48 \cdot 0$ & $52 \cdot 0$ & 37.5 & $150 \cdot 0$ & $(201 \cdot 2)$ & 144 & $71 \cdot 3$ & $67 \cdot 4$ & $49 \cdot 9$ & $57 \cdot 2$ & $(165 \cdot 1)$ & $(206 \cdot 7)$ \\
\hline Fish and seafood, fresh and frozen & $42 \cdot 2$ & $51 \cdot 0$ & $30 \cdot 0$ & $141 \cdot 9$ & $(200 \cdot 3)$ & 121 & $59 \cdot 9$ & $70 \cdot 5$ & $48 \cdot 4$ & $57 \cdot 5$ & $(150 \cdot 0)$ & $(201 \cdot 2)$ \\
\hline Fish and seafood, preserved & $5 \cdot 8$ & $11 \cdot 5$ & $0 \cdot 0$ & $31 \cdot 3$ & $(45 \cdot 2)$ & 60 & $29 \cdot 7$ & $19 \cdot 5$ & $13 \cdot 4$ & $17 \cdot 8$ & $(45 \cdot 1)$ & $(52 \cdot 1)$ \\
\hline Milk, milk products and substitutes & $188 \cdot 0$ & $129 \cdot 0$ & $176 \cdot 7$ & $405 \cdot 7$ & $(568 \cdot 3)$ & 200 & $99 \cdot 0$ & $189 \cdot 8$ & $128 \cdot 2$ & $178 \cdot 0$ & $412 \cdot 8$ & $(581 \cdot 7)$ \\
\hline $\begin{array}{l}\text { Milk, milk-based beverages, human milk, infant formula } \\
\text { and substitutes }\end{array}$ & $119 \cdot 7$ & $110 \cdot 5$ & $112 \cdot 5$ & $281 \cdot 9$ & $(407 \cdot 5)$ & 158 & $78 \cdot 2$ & $153 \cdot 0$ & $102 \cdot 4$ & $150 \cdot 0$ & $(323 \cdot 7)$ & $(500 \cdot 0)$ \\
\hline Yoghurt and fermented milk & $10 \cdot 6$ & $31 \cdot 0$ & $0 \cdot 0$ & $83 \cdot 3$ & $(125 \cdot 0)$ & 26 & $12 \cdot 9$ & $82 \cdot 4$ & $39 \cdot 9$ & $83 \cdot 3$ & $(125 \cdot 0)$ & $(150 \cdot 0)$ \\
\hline Cheese and substitutes & $57 \cdot 0$ & $39 \cdot 9$ & $51 \cdot 7$ & $133 \cdot 0$ & $(170 \cdot 7)$ & 195 & $96 \cdot 5$ & $59 \cdot 0$ & $39 \cdot 1$ & $52 \cdot 5$ & $134 \cdot 8$ & $(181 \cdot 1)$ \\
\hline Milk-based desserts and substitutes & $0 \cdot 7$ & $4 \cdot 8$ & $0 \cdot 0$ & $0 \cdot 0$ & $(31 \cdot 3)$ & 8 & $4 \cdot 0$ & $17 \cdot 9$ & $17 \cdot 3$ & $7 \cdot 6$ & $(50 \cdot 0)$ & $(50 \cdot 0)$ \\
\hline
\end{tabular}




\begin{tabular}{|c|c|c|c|c|c|c|c|c|c|c|c|c|}
\hline \multirow[b]{2}{*}{ Food categories } & \multicolumn{5}{|c|}{ Total population ( $n$ 202) } & \multicolumn{7}{|c|}{ Consumers } \\
\hline & Mean & SD & Median & 95 th $^{*}$ & 99th* & $n$ & $\%$ & Mean & SD & Median & 95th* & 99th* \\
\hline Oils and fats & $43 \cdot 8$ & $15 \cdot 6$ & $43 \cdot 8$ & $67 \cdot 8$ & $(90 \cdot 2)$ & 202 & $100 \cdot 0$ & $43 \cdot 8$ & $15 \cdot 6$ & $43 \cdot 8$ & $67 \cdot 8$ & $(90 \cdot 2)$ \\
\hline Olive oil & $37 \cdot 9$ & $14 \cdot 7$ & $36 \cdot 9$ & $62 \cdot 2$ & $(72 \cdot 9)$ & 202 & $100 \cdot 0$ & $37 \cdot 9$ & $14 \cdot 7$ & $36 \cdot 9$ & $62 \cdot 2$ & $(72 \cdot 9)$ \\
\hline Other vegetable oils & $1 \cdot 6$ & $3 \cdot 6$ & 0.0 & $7 \cdot 3$ & $(15 \cdot 0)$ & 52 & $25 \cdot 7$ & $6 \cdot 2$ & $4 \cdot 6$ & $5 \cdot 0$ & $(15 \cdot 0)$ & $(30 \cdot 0)$ \\
\hline Butter and creams & $3 \cdot 9$ & $5 \cdot 9$ & $0 \cdot 0$ & $16 \cdot 0$ & $(23 \cdot 7)$ & 99 & $49 \cdot 0$ & $7 \cdot 9$ & $6 \cdot 3$ & $6 \cdot 3$ & $(20 \cdot 5)$ & $(34 \cdot 6)$ \\
\hline Other fats & $0 \cdot 4$ & $1 \cdot 6$ & $0 \cdot 0$ & $3 \cdot 3$ & $(6 \cdot 7)$ & 21 & $10 \cdot 4$ & $4 \cdot 2$ & $3 \cdot 1$ & $3 \cdot 3$ & $(10 \cdot 7)$ & $(13 \cdot 1)$ \\
\hline Eggs & $23 \cdot 2$ & $26 \cdot 5$ & $16 \cdot 6$ & $72 \cdot 2$ & $(114 \cdot 6)$ & 151 & $74 \cdot 8$ & $31 \cdot 1$ & $26 \cdot 4$ & $23 \cdot 3$ & $(80 \cdot 1)$ & (133.7) \\
\hline Alcoholic beverages and substitutes & $177 \cdot 7$ & $172 \cdot 9$ & $160 \cdot 0$ & 480.0 & $(666 \cdot 8)$ & 177 & 87.6 & $202 \cdot 9$ & $170 \cdot 3$ & $180 \cdot 0$ & $560 \cdot 0$ & $(680 \cdot 1)$ \\
\hline Regular wine and substitutes & $164 \cdot 2$ & $158 \cdot 7$ & $160 \cdot 0$ & $480 \cdot 0$ & $(560 \cdot 0)$ & 174 & $86 \cdot 1$ & $190 \cdot 6$ & $155 \cdot 6$ & $166 \cdot 7$ & $480 \cdot 0$ & $(600 \cdot 0)$ \\
\hline Beer, cider and substitutes & $12 \cdot 1$ & $47 \cdot 0$ & $0 \cdot 0$ & $110 \cdot 0$ & $(220 \cdot 0)$ & 17 & $8 \cdot 4$ & $143 \cdot 8$ & $87 \cdot 6$ & $133 \cdot 3$ & $(400 \cdot 0)$ & $(400 \cdot 0)$ \\
\hline $\begin{array}{l}\text { Sweet wine, spumante, wine-based appetizers, } \\
\text { spirits and liquors }\end{array}$ & $1 \cdot 4$ & $6 \cdot 3$ & 0.0 & $13 \cdot 3$ & $(40 \cdot 0)$ & 26 & $12 \cdot 9$ & $11 \cdot 1$ & $14 \cdot 5$ & $3 \cdot 8$ & $(40 \cdot 0)$ & $(40 \cdot 0)$ \\
\hline Sweet products and substitutes & $28 \cdot 9$ & $29 \cdot 8$ & $22 \cdot 8$ & $82 \cdot 3$ & $(143 \cdot 3)$ & 183 & $90 \cdot 6$ & 31.9 & $29 \cdot 7$ & $24 \cdot 5$ & $82 \cdot 7$ & $(174 \cdot 7)$ \\
\hline Ice cream, ice lolly and substitutes & $5 \cdot 3$ & $21 \cdot 3$ & $0 \cdot 0$ & $33 \cdot 3$ & $(100 \cdot 0)$ & 20 & $9 \cdot 9$ & $53 \cdot 7$ & $45 \cdot 5$ & $33 \cdot 3$ & $(172 \cdot 9)$ & $(179 \cdot 2)$ \\
\hline Chocolate and substitutes & $1 \cdot 0$ & $3 \cdot 4$ & $0 \cdot 0$ & $6 \cdot 0$ & $(16 \cdot 7)$ & 29 & $14 \cdot 4$ & 6.9 & $6 \cdot 6$ & $4 \cdot 2$ & $(18 \cdot 0)$ & $(33 \cdot 3)$ \\
\hline Candies, jam and other sweet products (incl. sugar-free) & $3 \cdot 6$ & $10 \cdot 6$ & $0 \cdot 0$ & $25 \cdot 7$ & $(46 \cdot 7)$ & 37 & $18 \cdot 3$ & $19 \cdot 7$ & $17 \cdot 3$ & $15 \cdot 0$ & $(63 \cdot 3)$ & $(72 \cdot 9)$ \\
\hline Sugar, fructose, honey and other nutritious sweeteners & $18 \cdot 7$ & $16 \cdot 9$ & $16 \cdot 0$ & $46 \cdot 8$ & $(64 \cdot 7)$ & 170 & $84 \cdot 2$ & $22 \cdot 2$ & $16 \cdot 1$ & $18 \cdot 7$ & $49 \cdot 6$ & $(77 \cdot 3)$ \\
\hline Cocoa and cocoa-based powder & $0 \cdot 2$ & $2 \cdot 1$ & $0 \cdot 0$ & $0 \cdot 0$ & $(5 \cdot 1)$ & 9 & $4 \cdot 5$ & $5 \cdot 2$ & 8.9 & 1.9 & $(28 \cdot 0)$ & $(28 \cdot 0)$ \\
\hline Artificial sweeteners & $0 \cdot 1$ & 0.5 & $0 \cdot 0$ & $0 \cdot 1$ & $(2 \cdot 9)$ & 13 & $6 \cdot 4$ & $1 \cdot \overline{6}$ & 1.5 & $1 \cdot 3$ & $(4 \cdot 2)$ & $(4 \cdot 2)$ \\
\hline Meal substitutes & \multicolumn{5}{|c|}{ Not consumed } & 0 & $0 \cdot 0$ & \multicolumn{5}{|c|}{ Not consumed } \\
\hline Water and other non-alcoholic beverages & $762 \cdot 2$ & $388 \cdot 3$ & $722 \cdot 2$ & $1507 \cdot 9$ & $(2026 \cdot 7)$ & 202 & $100 \cdot 0$ & $762 \cdot 2$ & $388 \cdot 3$ & $722 \cdot 2$ & $1507 \cdot 9$ & $(2026 \cdot 7)$ \\
\hline Tap water (as such, in beverages or recipes) & $226 \cdot 5$ & $349 \cdot 8$ & $95 \cdot 0$ & $872 \cdot 1$ & $(1677 \cdot 9)$ & 126 & $62 \cdot 4$ & $363 \cdot 1$ & 383.0 & $238 \cdot 7$ & $(1260 \cdot 0)$ & $(1680 \cdot 0)$ \\
\hline Bottled water & $383 \cdot 8$ & $375 \cdot 1$ & $326 \cdot 7$ & $1013 \cdot 3$ & $(1333 \cdot 3)$ & 140 & $69 \cdot 3$ & $553 \cdot 7$ & $329 \cdot 6$ & $533 \cdot 3$ & $(1066 \cdot 7)$ & $(1653 \cdot 3)$ \\
\hline Coffee, tea, herbal tea and substitutes & $130 \cdot 1$ & $142 \cdot 4$ & $100 \cdot 0$ & $333 \cdot 3$ & $(540 \cdot 0)$ & 193 & $95 \cdot 5$ & $136 \cdot 2$ & $142 \cdot 8$ & $100 \cdot 0$ & $346 \cdot 7$ & $(560 \cdot 0)$ \\
\hline Fruit and vegetable juices & $15 \cdot 7$ & $50 \cdot 0$ & $0 \cdot 0$ & $133 \cdot 3$ & $(202 \cdot 9)$ & 92 & $45 \cdot 5$ & $34 \cdot 5$ & $69 \cdot 8$ & $4 \cdot 5$ & $(200 \cdot 0)$ & $(466 \cdot 7)$ \\
\hline Other soft drinks & $6 \cdot 1$ & $25 \cdot 4$ & $0 \cdot 0$ & $53 \cdot 3$ & $(120 \cdot 0)$ & 15 & $7 \cdot 4$ & $82 \cdot 7$ & $49 \cdot 7$ & $53 \cdot 3$ & $(213 \cdot 3)$ & $(213 \cdot 3)$ \\
\hline Miscellaneous & $3 \cdot 2$ & $4 \cdot 0$ & $2 \cdot 2$ & $10 \cdot 0$ & $(16 \cdot 0)$ & 159 & $78 \cdot 7$ & $4 \cdot 1$ & $4 \cdot 1$ & $2 \cdot 9$ & $(12 \cdot 0)$ & $(20 \cdot 4)$ \\
\hline Total amount of foods and beveragest & $2255 \cdot 7$ & $587 \cdot 6$ & $2165 \cdot 3$ & $3231 \cdot 2$ & $(3817 \cdot 3)$ & 202 & $100 \cdot 0$ & $2255 \cdot 7$ & $587 \cdot 6$ & $2165 \cdot 3$ & $3231 \cdot 2$ & $(3817 \cdot 3)$ \\
\hline Total amount of liquid foods & $1061 \cdot 5$ & 394.9 & $1010 \cdot 3$ & $1843 \cdot 8$ & $(2143 \cdot 3)$ & 202 & $100 \cdot 0$ & $1061 \cdot 5$ & 394.9 & $1010 \cdot 3$ & $1843 \cdot 8$ & $(2143 \cdot 3)$ \\
\hline Total amount of solid foods & $1194 \cdot 2$ & $313 \cdot 1$ & $1176 \cdot 0$ & $1662 \cdot 0$ & $(2230 \cdot 1)$ & 202 & $100 \cdot 0$ & $1194 \cdot 2$ & $313 \cdot 1$ & $1176 \cdot 0$ & $1662 \cdot 0$ & $(2230 \cdot 1)$ \\
\hline
\end{tabular}

*High percentiles of consumption assessed on the basis of a $3 \mathrm{~d}$ survey provide an overestimate of long-term high levels of consumption. Values are enclosed in parentheses when the number of subjects to which they

refer is lower than 160 and 800 for the 95th and 99th percentile, respectively, since these values bear a large uncertainty and provide only a rough indication of high levels of consumption. 
Table 11 Mean, standard deviation, median and high percentiles of individual daily consumption ( $3 \mathrm{~d}$ average) by food category in the total population and in consumers ( $\mathrm{g} / \mathrm{d}$ ) - elderly ( $\geq 65$ years), females: Italian National Food Consumption Survey INRAN-SCAI 2005-06

\begin{tabular}{|c|c|c|c|c|c|c|c|c|c|c|c|c|}
\hline \multirow[b]{2}{*}{ Food categories } & \multicolumn{5}{|c|}{ Total population $(n 316)$} & \multicolumn{7}{|c|}{ Consumers } \\
\hline & Mean & SD & Median & 95th* & 99th* & $n$ & $\%$ & Mean & SD & Median & 95th* & 99th* \\
\hline Cereals, cereal products and substitutes & $228 \cdot 3$ & $91 \cdot 6$ & $220 \cdot 7$ & $386 \cdot 9$ & $(519 \cdot 8)$ & 316 & $100 \cdot 0$ & $228 \cdot 3$ & $91 \cdot 6$ & $220 \cdot 7$ & $386 \cdot 9$ & $(519 \cdot 8)$ \\
\hline Bread & $102 \cdot 1$ & $73 \cdot 6$ & $90 \cdot 0$ & $240 \cdot 0$ & $(315 \cdot 0)$ & 292 & $92 \cdot 4$ & $110 \cdot 5$ & $70 \cdot 2$ & $90 \cdot 3$ & $240 \cdot 0$ & $(320 \cdot 0)$ \\
\hline Pasta and pasta substitutes & $50 \cdot 7$ & $28 \cdot 6$ & $49 \cdot 9$ & $100 \cdot 6$ & $(117 \cdot 4)$ & 294 & $93 \cdot 0$ & $54 \cdot 5$ & $25 \cdot 9$ & $52 \cdot 5$ & $100 \cdot 9$ & $(121 \cdot 5)$ \\
\hline Pizza & $4 \cdot 1$ & $17 \cdot 5$ & $0 \cdot 0$ & $25 \cdot 0$ & $(116 \cdot 7)$ & 25 & $7 \cdot 9$ & $52 \cdot 3$ & $37 \cdot 6$ & $33 \cdot 3$ & $(125 \cdot 0)$ & $(150 \cdot 0)$ \\
\hline Rice & $17 \cdot 9$ & $30 \cdot 0$ & $0 \cdot 0$ & $83 \cdot 3$ & $(166 \cdot 7)$ & 136 & $43 \cdot 0$ & $41 \cdot 6$ & $33 \cdot 2$ & $30 \cdot 4$ & $(104 \cdot 2)$ & $(166 \cdot 7)$ \\
\hline Wheat, other cereals and flours & $18 \cdot 9$ & $22 \cdot 2$ & $9 \cdot 8$ & $61 \cdot 6$ & $(90 \cdot 0)$ & 232 & $73 \cdot 4$ & $25 \cdot 8$ & $22 \cdot 2$ & $19 \cdot 3$ & $64 \cdot 3$ & $(97 \cdot 7)$ \\
\hline Breakfast cereals & $0 \cdot 7$ & $3 \cdot 9$ & $0 \cdot 0$ & $0 \cdot 0$ & $(25 \cdot 0)$ & 14 & $4 \cdot 4$ & $16 \cdot 9$ & $9 \cdot 1$ & $15 \cdot 0$ & $(30 \cdot 0)$ & $(30 \cdot 0)$ \\
\hline Biscuits & $12 \cdot 3$ & $16 \cdot 3$ & $5 \cdot 2$ & $46 \cdot 3$ & $(66 \cdot 7)$ & 163 & $51 \cdot 6$ & $23 \cdot 9$ & $15 \cdot 4$ & $20 \cdot 0$ & $53 \cdot 3$ & $(78 \cdot 8)$ \\
\hline Savoury fine bakery products & $11 \cdot 7$ & $18 \cdot 6$ & $0 \cdot 0$ & $43 \cdot 3$ & $(83 \cdot 3)$ & 147 & $46 \cdot 5$ & $25 \cdot 2$ & $20 \cdot 1$ & $20 \cdot 9$ & $(66 \cdot 7)$ & $(102 \cdot 7)$ \\
\hline Cakes and sweet snacks & $9 \cdot 7$ & $19 \cdot 8$ & $0 \cdot 0$ & $52 \cdot 1$ & $(83 \cdot 3)$ & 85 & $26 \cdot 9$ & $36 \cdot 1$ & $22 \cdot 6$ & $34 \cdot 1$ & $(73 \cdot 6)$ & $(136 \cdot 1)$ \\
\hline Pulses, fresh and processed & $9 \cdot 9$ & $18 \cdot 9$ & 0.0 & $52 \cdot 5$ & $(77 \cdot 1)$ & 104 & 32.9 & $30 \cdot 2$ & $21 \cdot 7$ & $23 \cdot 2$ & $(73.5)$ & $(97 \cdot 2)$ \\
\hline Vegetables, fresh and processed & $210 \cdot 6$ & $108 \cdot 9$ & $192 \cdot 8$ & $413 \cdot 7$ & $(499 \cdot 8)$ & 314 & $99 \cdot 4$ & $211 \cdot 9$ & $107 \cdot 9$ & $193 \cdot 8$ & $413 \cdot 7$ & $(499 \cdot 8)$ \\
\hline Leafy vegetables, fresh & $50 \cdot 8$ & $56 \cdot 1$ & $33 \cdot 8$ & $153 \cdot 6$ & $(263 \cdot 3)$ & 281 & 88.9 & $57 \cdot 1$ & $56 \cdot 4$ & $40 \cdot 5$ & $166 \cdot 7$ & $(272 \cdot 2)$ \\
\hline Tomatoes, fresh & $36 \cdot 6$ & $38 \cdot 3$ & $25 \cdot 0$ & $116 \cdot 3$ & $(149 \cdot 0)$ & 244 & $77 \cdot 2$ & $47 \cdot 4$ & $37 \cdot 2$ & $39 \cdot 1$ & $117 \cdot 7$ & $(150 \cdot 0)$ \\
\hline Other fruiting vegetables, fresh & $32 \cdot 4$ & $50 \cdot 0$ & $8 \cdot 1$ & $147 \cdot 0$ & $(231 \cdot 3)$ & 200 & $63 \cdot 3$ & $51 \cdot 2$ & $54 \cdot 7$ & $34 \cdot 3$ & $172 \cdot 5$ & $(249 \cdot 7)$ \\
\hline Roots and onions, fresh & $20 \cdot 8$ & $24 \cdot 5$ & $11 \cdot 6$ & $77 \cdot 6$ & $(108 \cdot 8)$ & 308 & $97 \cdot 5$ & $21 \cdot 3$ & $24 \cdot 6$ & $12 \cdot 4$ & $77 \cdot 6$ & $(108 \cdot 8)$ \\
\hline Other vegetables, fresh & $36 \cdot 9$ & $48 \cdot 9$ & $15 \cdot 8$ & $131 \cdot 4$ & $(229 \cdot 2)$ & 264 & $83 \cdot 5$ & $44 \cdot 2$ & $50 \cdot 4$ & $26 \cdot 2$ & $141 \cdot 3$ & $(235 \cdot 3)$ \\
\hline Vegetables, processed & $31 \cdot 5$ & $29 \cdot 6$ & $31 \cdot 5$ & $84 \cdot 0$ & $(128.9)$ & 240 & $75 \cdot 9$ & $41 \cdot \overline{4}$ & $27 \cdot 2$ & $33 \cdot \overline{4}$ & $92 \cdot 8$ & $(137 \cdot 1)$ \\
\hline Spices and Hherbs & $1 \cdot 6$ & $2 \cdot 6$ & $0 \cdot 8$ & $6 \cdot 1$ & $(8 \cdot 9)$ & 248 & $78 \cdot 5$ & $2 \cdot 1$ & $2 \cdot 8$ & $1 \cdot 2$ & $6 \cdot 5$ & $(8 \cdot 9)$ \\
\hline Potatoes, tubers and their products & $50 \cdot 8$ & $55 \cdot 0$ & $41 \cdot 5$ & $155 \cdot 8$ & $(238 \cdot 3)$ & 228 & $72 \cdot 2$ & $70 \cdot 3$ & $53 \cdot 1$ & $58 \cdot 6$ & $166 \cdot 7$ & $(244 \cdot 7)$ \\
\hline Fruit, fresh and processed & $268 \cdot 3$ & $165 \cdot 9$ & $241 \cdot 6$ & $564 \cdot 0$ & $(753 \cdot 3)$ & 305 & 96.5 & $278 \cdot 0$ & $160 \cdot 7$ & $250 \cdot 0$ & $564 \cdot 0$ & $(753 \cdot 3)$ \\
\hline Citrus fruit, fresh & $54 \cdot 5$ & $97 \cdot 2$ & $0 \cdot 0$ & $242 \cdot 7$ & $(421 \cdot 3)$ & 156 & $49 \cdot 4$ & $110 \cdot 4$ & 113.9 & $68 \cdot 7$ & $(366 \cdot 0)$ & $(640 \cdot 0)$ \\
\hline Exotic fruit, fresh & $11 \cdot 9$ & $22 \cdot 2$ & $0 \cdot 0$ & $66 \cdot 7$ & $(100 \cdot 0)$ & 96 & $30 \cdot 4$ & $39 \cdot 0$ & $23 \cdot 6$ & $33 \cdot 3$ & $(100 \cdot 0)$ & $(106 \cdot 7)$ \\
\hline Other fruit, fresh & $199 \cdot 7$ & $150 \cdot 5$ & $168 \cdot 0$ & $476 \cdot 0$ & $(695 \cdot 3)$ & 290 & $91 \cdot 8$ & $217 \cdot 7$ & $144 \cdot 2$ & $178 \cdot 7$ & $489 \cdot 3$ & $(723 \cdot 0)$ \\
\hline Nuts, seeds, olives and their products, dried fruit & $2 \cdot 2$ & 6.5 & 0.0 & $13 \cdot 3$ & $(21 \cdot 4)$ & 72 & $22 \cdot 8$ & $9 \cdot 7$ & $10 \cdot 7$ & $7 \cdot 4$ & $(21 \cdot 4)$ & $(64 \cdot 0)$ \\
\hline Other processed fruit (in syrup, in purée, etc.) & 0.0 & $0 \cdot 1$ & $0 \cdot 0$ & $0 \cdot 0$ & $(0 \cdot 0)$ & 3 & 0.9 & $1 \cdot 3$ & $0 \cdot 6$ & $1 \cdot 3$ & $(1.9)$ & $(1.9)$ \\
\hline Meat, meat products and substitutes & $88 \cdot 0$ & $46 \cdot 0$ & $83 \cdot 2$ & $171 \cdot 6$ & $(224 \cdot 9)$ & 308 & 97.5 & $90 \cdot 3$ & $44 \cdot 3$ & $84 \cdot 2$ & $171 \cdot 6$ & $(224 \cdot 9)$ \\
\hline Beef and veal, not preserved, excl. offal & $35 \cdot 2$ & $31 \cdot 6$ & $33 \cdot 3$ & 96.9 & $(124 \cdot 1)$ & 228 & $72 \cdot 2$ & $48 \cdot 8$ & $26 \cdot 8$ & 41.9 & $99 \cdot 0$ & $(133.0)$ \\
\hline Pork, not preserved, excl. offal & $7 \cdot 2$ & $16 \cdot 4$ & $0 \cdot 0$ & $46 \cdot 9$ & $(58 \cdot 6)$ & 76 & $24 \cdot 1$ & $30 \cdot 0$ & $21 \cdot 0$ & $33 \cdot 5$ & $(58 \cdot 6)$ & $(93 \cdot 8)$ \\
\hline Poultry and game, not preserved, excl. offal & $22 \cdot 0$ & $28 \cdot 8$ & $0 \cdot 0$ & $83 \cdot 4$ & $(103 \cdot 7)$ & 145 & $45 \cdot 9$ & $47 \cdot 9$ & $23 \cdot 8$ & 38.9 & $(98.9)$ & $(135 \cdot 4)$ \\
\hline Other meats, not preserved, excl. offal & $5 \cdot 2$ & $18 \cdot 5$ & 0.0 & $49 \cdot 4$ & $(105 \cdot 2)$ & 29 & $9 \cdot 2$ & $57 \cdot 0$ & $28 \cdot 6$ & $49 \cdot 4$ & $(115 \cdot 2)$ & $(120 \cdot 8)$ \\
\hline $\begin{array}{l}\text { Ham, salami, sausages and other preserved meats, } \\
\text { excl. offal }\end{array}$ & $17 \cdot 6$ & $20 \cdot 4$ & $13 \cdot 3$ & $58 \cdot 7$ & $(91 \cdot 7)$ & 218 & $69 \cdot 0$ & $25 \cdot 6$ & $20 \cdot 0$ & $20 \cdot 0$ & $71 \cdot 8$ & $(100 \cdot 0)$ \\
\hline Offal, blood and their products & 0.7 & 4.9 & $0 \cdot 0$ & 0.0 & $(25 \cdot 0)$ & 8 & $2 \cdot 5$ & $25 \cdot 8$ & $18 \cdot 0$ & $25 \cdot 0$ & $(50 \cdot 0)$ & $(50 \cdot 0)$ \\
\hline Meat substitutes & $0 \cdot 1$ & 1.9 & $0 \cdot 0$ & $0 \cdot 0$ & $(0.0)$ & 1 & $0 \cdot 3$ & $33 \cdot 3$ & - & $33 \cdot 3$ & $(33 \cdot 3)$ & $(33.3)$ \\
\hline Fish, seafood and their products & 32.9 & $39 \cdot 6$ & $16 \cdot 7$ & $110 \cdot 0$ & $(137 \cdot 7)$ & 185 & $58 \cdot 5$ & $56 \cdot 2$ & $37 \cdot 1$ & $50 \cdot 0$ & $120 \cdot 9$ & $(200 \cdot 3)$ \\
\hline Fish and seafood, fresh and frozen & $28 \cdot 6$ & $37 \cdot 8$ & 0.0 & $108 \cdot 0$ & $(132 \cdot 0)$ & 152 & $48 \cdot 1$ & $59 \cdot \overline{5}$ & $33 \cdot 6$ & $50 \cdot 0$ & $(120 \cdot 9)$ & $(196 \cdot 3)$ \\
\hline Fish and seafood, preserved & $4 \cdot 3$ & $9 \cdot 8$ & 0.0 & $26 \cdot 5$ & $(47 \cdot 8)$ & 78 & $24 \cdot 7$ & $17 \cdot 4$ & $12 \cdot 8$ & $16 \cdot 7$ & $(47 \cdot 8)$ & $(58.4)$ \\
\hline Milk, milk products and substitutes & $199 \cdot 6$ & $125 \cdot 4$ & $195 \cdot 0$ & $421 \cdot 1$ & $(480 \cdot 1)$ & 313 & $99 \cdot 1$ & $201 \cdot 5$ & $124 \cdot 4$ & $196 \cdot 3$ & $421 \cdot 1$ & $(480 \cdot 1)$ \\
\hline $\begin{array}{l}\text { Milk, milk-based beverages, human milk, infant formula } \\
\text { and substitutes }\end{array}$ & $129 \cdot 9$ & $106 \cdot 0$ & $135 \cdot 0$ & $314 \cdot 0$ & $(377 \cdot 6)$ & 254 & $80 \cdot 4$ & $161 \cdot 6$ & $94 \cdot 0$ & $165 \cdot 0$ & $330 \cdot 0$ & $(395 \cdot 4)$ \\
\hline Yoghurt and fermented milk & $18 \cdot 9$ & $48 \cdot 5$ & $0 \cdot 0$ & $125 \cdot 0$ & $(166 \cdot 7)$ & 68 & $21 \cdot 5$ & $87 \cdot 9$ & $69 \cdot 9$ & $83 \cdot 3$ & $(166 \cdot 7)$ & $(531 \cdot 7)$ \\
\hline Cheese and substitutes & $49 \cdot 9$ & $40 \cdot 1$ & $42 \cdot 6$ & $122 \cdot 2$ & $(171 \cdot 2)$ & 305 & $96 \cdot 5$ & $51 \cdot 7$ & $39 \cdot 7$ & $43 \cdot 7$ & $122 \cdot 2$ & $(171 \cdot 2)$ \\
\hline Milk-based desserts and substitutes & 0.9 & $6 \cdot 7$ & $0 \cdot 0$ & $0 \cdot 0$ & $(33 \cdot 3)$ & 10 & 3.2 & $27 \cdot 8$ & $26 \cdot 9$ & $20 \cdot 8$ & $(83 \cdot 3)$ & $(83 \cdot 3)$ \\
\hline
\end{tabular}




\begin{tabular}{|c|c|c|c|c|c|c|c|c|c|c|c|c|}
\hline \multirow[b]{2}{*}{ Food categories } & \multicolumn{5}{|c|}{ Total population ( $n$ 316) } & \multicolumn{7}{|c|}{ Consumers } \\
\hline & Mean & SD & Median & $95 t^{\star}$ & 99th* & $n$ & $\%$ & Mean & SD & Median & 95th* & 99th* \\
\hline Oils and fats & $34 \cdot 3$ & $14 \cdot 1$ & $31 \cdot 9$ & $59 \cdot 3$ & $(77 \cdot 9)$ & 315 & $99 \cdot 7$ & $34 \cdot 4$ & $14 \cdot 0$ & $32 \cdot 0$ & $59 \cdot 3$ & $(77 \cdot 9)$ \\
\hline Olive oil & $29 \cdot 3$ & $12 \cdot \cdot 4$ & $28 \cdot 2$ & $51 \cdot 9$ & $(62 \cdot 8)$ & 315 & $99 \cdot 7$ & $29 \cdot 4$ & $12 \cdot 3$ & $28 \cdot 2$ & $51 \cdot 9$ & $(62 \cdot 8)$ \\
\hline Other vegetable oils & 0.9 & $2 \cdot 5$ & 0.0 & $6 \cdot 1$ & $(10 \cdot 0)$ & 55 & $17 \cdot 4$ & $5 \cdot 4$ & $3 \cdot 5$ & $4 \cdot 7$ & $(10 \cdot 0)$ & $(25 \cdot 5)$ \\
\hline Butter and creams & $3 \cdot 6$ & $5 \cdot 8$ & 0.0 & $15 \cdot 6$ & $(25 \cdot 6)$ & 146 & $46 \cdot 2$ & $7 \cdot 8$ & $6 \cdot 4$ & $5 \cdot 7$ & $(21 \cdot 3)$ & $(30 \cdot 6)$ \\
\hline Other fats & $0 \cdot 4$ & $2 \cdot 1$ & $0 \cdot 0$ & $2 \cdot 7$ & $(12 \cdot 2)$ & 24 & $7 \cdot 6$ & $5 \cdot 7$ & $5 \cdot 2$ & $2 \cdot 8$ & $(16 \cdot 0)$ & $(16 \cdot 4)$ \\
\hline Eggs & $18 \cdot 8$ & $19 \cdot 7$ & $13 \cdot 1$ & $54 \cdot 3$ & $(71 \cdot 6)$ & 224 & $70 \cdot 9$ & $26 \cdot 5$ & $18 \cdot 5$ & $20 \cdot 4$ & $58 \cdot 6$ & $(77 \cdot 7)$ \\
\hline Alcoholic beverages and substitutes & $60 \cdot 2$ & $98 \cdot 1$ & $0 \cdot 0$ & $240 \cdot 1$ & $(420 \cdot 0)$ & 210 & $66 \cdot 5$ & $90 \cdot 6$ & $108 \cdot 4$ & $60 \cdot 0$ & $300 \cdot 0$ & $(453 \cdot 3)$ \\
\hline Regular wine and substitutes & $56 \cdot 6$ & $95 \cdot 5$ & 0.0 & $240 \cdot 1$ & $(420 \cdot 0)$ & 203 & $64 \cdot 2$ & $88 \cdot 1$ & $106 \cdot 9$ & $60 \cdot 0$ & $280 \cdot 0$ & $(453 \cdot 3)$ \\
\hline Beer, cider and substitutes & $3 \cdot 2$ & $20 \cdot 7$ & $0 \cdot 0$ & $0 \cdot 0$ & $(110 \cdot 0)$ & 9 & $2 \cdot 8$ & $110 \cdot 7$ & $59 \cdot 1$ & $110 \cdot 0$ & $(240 \cdot 0)$ & $(240 \cdot 0)$ \\
\hline $\begin{array}{l}\text { Sweet wine, spumante, wine-based appetizers, } \\
\text { spirits and liquors }\end{array}$ & 0.4 & $2 \cdot 9$ & 0.0 & $0 \cdot 1$ & $(13 \cdot 3)$ & 23 & $7 \cdot 3$ & $5 \cdot 6$ & $9 \cdot 4$ & $0 \cdot 7$ & $(16 \cdot 7)$ & $(40 \cdot 0)$ \\
\hline Sweet products and substitutes & $26 \cdot 8$ & $26 \cdot 1$ & $21 \cdot 2$ & $81 \cdot 8$ & $(121 \cdot 7)$ & 281 & $88 \cdot 9$ & $30 \cdot 2$ & $25 \cdot 7$ & $24 \cdot 0$ & $82 \cdot 3$ & $(127 \cdot 7)$ \\
\hline Ice cream, ice lolly and substitutes & $5 \cdot 8$ & $18 \cdot 7$ & $0 \cdot 0$ & $44 \cdot 0$ & $(83 \cdot 3)$ & 39 & $12 \cdot 3$ & $47 \cdot 0$ & $30 \cdot 0$ & $33 \cdot 3$ & $(123 \cdot 3)$ & $(153 \cdot 3)$ \\
\hline Chocolate and substitutes & 0.9 & $5 \cdot 1$ & 0.0 & $5 \cdot 1$ & $(17 \cdot 4)$ & 34 & $10 \cdot 8$ & $8 \cdot 4$ & $13 \cdot 6$ & $4 \cdot 3$ & $(26 \cdot 5)$ & $(78 \cdot 0)$ \\
\hline $\begin{array}{l}\text { Candies, jam and other sweet products } \\
\text { (incl. sugar-free) }\end{array}$ & $2 \cdot 9$ & $7 \cdot 5$ & 0.0 & $20 \cdot 0$ & $(33 \cdot 3)$ & 74 & $23 \cdot 4$ & $12 \cdot 4$ & $11 \cdot 0$ & $9 \cdot 7$ & $(33 \cdot 3)$ & $(50 \cdot 0)$ \\
\hline $\begin{array}{l}\text { Sugar, fructose, honey and other nutritious } \\
\text { sweeteners }\end{array}$ & $16 \cdot 8$ & $14 \cdot 1$ & $16 \cdot 0$ & $40 \cdot 0$ & $(61 \cdot 3)$ & 257 & $81 \cdot 3$ & $20 \cdot 6$ & $12 \cdot 8$ & $18 \cdot 7$ & $44 \cdot 0$ & $(64 \cdot 3)$ \\
\hline Cocoa and cocoa-based powder & 0.4 & $2 \cdot 0$ & 0.0 & $0 \cdot 8$ & $(11 \cdot 1)$ & 18 & $5 \cdot 7$ & $7 \cdot 0$ & $5 \cdot 2$ & $5 \cdot 8$ & $(19 \cdot 3)$ & $(19 \cdot 3)$ \\
\hline Artificial sweeteners & $0 \cdot 0$ & $0 \cdot 3$ & 0.0 & $0 \cdot 1$ & $(1 \cdot 3)$ & 21 & $6 \cdot 6$ & 0.7 & $0 \cdot 7$ & 0.4 & $(2 \cdot 0)$ & $(2 \cdot 5)$ \\
\hline Meal substitutes & \multicolumn{2}{|c|}{ Not consumed } & & & & 0 & $0 \cdot 0$ & & & & & \\
\hline Water and other non-alcoholic beverages & $807 \cdot 9$ & $339 \cdot 2$ & $771 \cdot 1$ & $1450 \cdot 0$ & $(1864 \cdot 4)$ & 316 & $100 \cdot 0$ & $807 \cdot 9$ & $339 \cdot 2$ & $771 \cdot 1$ & $1450 \cdot 0$ & $(1864 \cdot 4)$ \\
\hline Tap water (as such, in beverages or recipes) & $267 \cdot 9$ & $329 \cdot 9$ & $142 \cdot 5$ & $949 \cdot 3$ & $(1405 \cdot 0)$ & 221 & $69 \cdot 9$ & $383 \cdot 1$ & 333.9 & $285 \cdot 0$ & $1013 \cdot 3$ & $(1440 \cdot 0)$ \\
\hline Bottled water & 374.9 & $353 \cdot 0$ & $360 \cdot 0$ & $960 \cdot 0$ & $(1280 \cdot 0)$ & 213 & $67 \cdot 4$ & $556 \cdot 2$ & $289 \cdot 5$ & $533 \cdot 3$ & $1120 \cdot 0$ & $(1333 \cdot 3)$ \\
\hline Coffee, tea, herbal tea and substitutes & $130 \cdot 3$ & $111 \cdot 3$ & $102 \cdot 5$ & $360 \cdot 0$ & $(500 \cdot 0)$ & 296 & $93 \cdot 7$ & $139 \cdot 1$ & $109 \cdot 6$ & $112 \cdot 5$ & $365 \cdot 0$ & $(506 \cdot 7)$ \\
\hline Fruit and vegetable juices & $26 \cdot 3$ & $67 \cdot 5$ & 0.0 & $166 \cdot 7$ & $(268 \cdot 8)$ & 150 & $47 \cdot 5$ & $55 \cdot 5$ & $89 \cdot 5$ & $8 \cdot 0$ & $(250 \cdot 0)$ & $(473 \cdot 1)$ \\
\hline Other soft drinks & $8 \cdot 5$ & $41 \cdot 3$ & 0.0 & $53 \cdot 3$ & $(240 \cdot 0)$ & 20 & $6 \cdot 3$ & $134 \cdot 2$ & $102 \cdot 9$ & $106 \cdot 7$ & $(353 \cdot 3)$ & $(386 \cdot 7)$ \\
\hline Miscellaneous & $2 \cdot 6$ & $3 \cdot 3$ & $1 \cdot 3$ & $8 \cdot 4$ & $(15 \cdot 3)$ & 259 & $82 \cdot 0$ & $3 \cdot 2$ & $3 \cdot 4$ & $2 \cdot 3$ & 8.6 & $(19 \cdot 5)$ \\
\hline Total amount of foods and beverages $t$ & $2039 \cdot 6$ & $521 \cdot 9$ & $2011 \cdot 5$ & 3028.5 & $(3302 \cdot 6)$ & 316 & $100 \cdot 0$ & $2039 \cdot 6$ & $521 \cdot 9$ & $2011 \cdot 5$ & $3028 \cdot 5$ & $(3302 \cdot 6)$ \\
\hline Total amount of liquid foods & $1000 \cdot 0$ & $354 \cdot 2$ & $963 \cdot 6$ & $1683 \cdot 7$ & $(2058 \cdot 7)$ & 316 & $100 \cdot 0$ & $1000 \cdot 0$ & $354 \cdot 2$ & $963 \cdot 6$ & $1683 \cdot 7$ & $(2058 \cdot 7)$ \\
\hline Total amount of solid foods & $1039 \cdot 2$ & $293 \cdot 7$ & $1012 \cdot 2$ & $1551 \cdot 7$ & $(1895 \cdot 1)$ & 316 & $100 \cdot 0$ & $1039 \cdot 2$ & $293 \cdot 7$ & $1012 \cdot 2$ & $1551 \cdot 7$ & $(1895 \cdot 1)$ \\
\hline
\end{tabular}

${ }^{*}$ High percentiles of consumption assessed on the basis of a $3 \mathrm{~d}$ survey provide an overestimate of long-term high levels of consumption. Values are enclosed in parentheses when the number of subjects to which they refer is lower than 160 and 800 for the 95th and 99th percentile, respectively, since these values bear a large uncertainty and provide only a rough indication of high levels of consumption.

tAll beverages and milk in liquid state were classified as liquid foods; all other items were classified as solid foods. 




Fig. 2 Consumption pattern by age and sex: percentage contribution (in weight) of each food category ${ }^{*}$ to the total amount of food and beverages in the Italian National Food Consumption Survey INRAN-SCAI 2005-06.

*The contribution of 'Meal substitutes' and of 'Miscellaneous' is not reported. It was less than $0.2 \%$ in all age and sex classes. Statistically significant differences $(P<0.05$; Kruskal-Wallis test) were found among age classes in all reported food categories, except for 'Fish, seafood and their products' in males and for 'Pulses, fresh and processed' in males and females. Statistically significant differences between sex $(P<0.05$; Kruskal-Wallis test) were found only among adults and the elderly: in adults for 'Cereals, cereals products and substitutes', 'Fruit, fresh and processed', 'Meat, meat products and substitutes', 'Milk, milk products and substitutes', 'Oils and fats', 'Alcoholic beverages and substitutes', 'Eggs' and 'Water and other non alcoholic beverages'; in the elderly for 'Cereals, cereals products and substitutes', 'Fruit, fresh and processed', 'Meat, meat products and substitutes', 'Fish, seafood and their products', 'Milk, milk products and substitutes', 'Oils and fats', 'Alcoholic beverages and substitutes' and 'Water and other non-alcoholic beverages'

under-reporting. Individual dietary surveys conducted in European countries from 1980 to 2001 show participation rates varying from $36 \%$ to $86 \%{ }^{(13)}$. More recent surveys performed among adults in Ireland ${ }^{(14)}$, The Netherlands ${ }^{(15)}$ and the $\mathrm{UK}^{(16)}$ showed a participation rate of respectively $63 \%, 42 \%$ and $63 \%$.

The low participation rate could have affected the representativity of the study sample. However, the low percentage of subjects who declared to follow a specific diet during the food survey (6\%) suggests that no important selection of health-conscious subjects occurred. Moreover, the distribution in age and sex classes compares well with the segmentation of the Italian population in 2006 as described by ISTAT $^{(17)}$. Females are slightly over-represented ( $55 \% v .51 \%$ according to ISTAT), whereas children aged less than 3 years of age ( $2 \% v .4 \%$ according to ISTAT) and elderly subjects ( $16 \% v$ v. 20\% according to ISTAT) are slightly under-represented. The education level of the study sample is high, with $56 \%$ of subjects aged 11 years or more having a high school or university degree $v .35 \%$ the Italian population as described by $\operatorname{ISTAT}^{(18)}$.

\section{Dietary assessment technique}

The INRAN-DIARIO3.1 ${ }^{(9)}$ management system permitted a multi-operator data entry and food codification and at the same time ensured reliable data and a high level of standardization of the field workers' procedures.

The fact that the field workers met each subject three times and carefully checked every single entry helped to reduce errors such as misreporting and omissions. How-

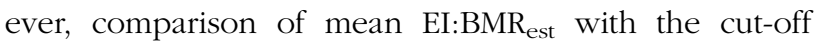
values derived by Goldberg et al. ${ }^{(19)}$ for adults $(99 \cdot 7 \%$ confidence interval) suggests that a certain level of underreporting occurred in this sample and in particular in males. It is noteworthy that these cut-off values are based on the assumption of a PAL of 1.55 , which includes desirable physical activity for sedentary adults ${ }^{(10)}$. It may therefore overestimate effective PAL of the adult Italian population. Moreover, EI:BMR ${ }_{\text {est }}$ was lower in adult males, where a higher proportion of obese subjects were found. This may be due to the notorious higher degree of under-reporting in obese individuals but also to an overestimate of their BMR, leading to an overestimate of under-reporting. In fact, $\mathrm{BMR}_{\mathrm{est}}$ based on current body weight may overestimate true BMR in obese individuals since fat body mass is less metabolically active.

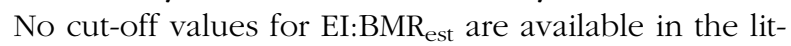
erature for the other age classes of the study sample. The observed EI:EE in elderly males and in infants, children and teenagers of both sexes ranged from 0.98 to 1.04 
(Table 2), suggesting that no gross under-reporting occurred in these groups. The highest apparent level of under-reporting was in adult males and females and in elderly females.

In the absence of information on the individual activity level of subjects that would allow us to identify underreporters, no subject was excluded from further analysis. The same decision was taken in the national survey conducted in Ireland in 1997-99(20), in which a comparable mean

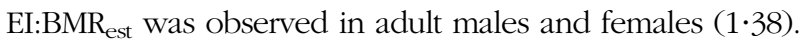

\section{Food classification}

A detailed list of food items included in each category is provided in the Appendix to ensure a correct interpretation of data.

A number of cereal products such as plain pizza with tomato and biscuits were classified as cereal products, whereas they also contain other ingredients (fats, eggs, tomatoes, sugar, etc.). Other cereal products (e.g. pizza with a variety of ingredients, most cakes, some fresh pasta and gnocchi) were handled as recipes and disaggregated into flour and other ingredients. Therefore, consumption values reported in the subcategories 'Pizza', 'Pasta' and 'Cakes and sweet snacks' are underestimates. On the other hand, when food items are classified in a subcategory such as 'Biscuits', this leads to an underestimate of the consumption of single ingredients such as flour and sugar in their respective food categories.

The subcategory 'Sugar' is related to discretionary sugar (e.g. added by the respondents in coffee) plus sugar from home-made recipes. It does not include sugar present in composite foods such as soft drinks.

Data reported in the category 'Oils and fats' include discretionary fats added in a large number of recipes but do not include fats present in a number of composite foods. In Italy, each recipe is traditionally prepared with one specific fat: risotto would be prepared with butter, whereas most sauces used with pasta would be prepared with olive oil. Subjects were asked to report the main ingredients of the recipes on the diaries and standard recipes would generally be used. The proportion of olive oil among vegetable oils in the present survey (93\%) has probably been slightly overestimated. According to data from the Institute for Agricultural Market Studies (ISMEA) $^{(21)}$, in the year 2004 , olive oil represented $84 \%$ of the total household purchases of vegetable fats in Italy.

\section{Food consumption pattern}

Detailed analyses of the present survey in relation to public health nutrition issues will be performed in the future. As a first step, some of the results can be compared with those population goals established for the prevention of chronic diseases which are expressed in terms of food.

The overall individual consumption of fruit and vegetables in the whole study sample was $208 \mathrm{~g} / \mathrm{d}$ and $210 \mathrm{~g} / \mathrm{d}$, respectively, meeting the minimum population goal of $400 \mathrm{~g}$ of fruit and vegetables daily established by $\mathrm{FAO} / \mathrm{WHO}^{(22)}$.

A goal was recently set for the population average consumption of red meat (beef, pork, lamb and goat from domesticated animals, including that contained in processed foods): it should be less than $300 \mathrm{~g} /$ week as cooked meat (approximately $400-450 \mathrm{~g}$ as raw weight) for the prevention of colorectal cancer ${ }^{(23)}$. Overall consumption of red meat in the study sample was obtained by adding up fresh beef and veal $(42.7 \mathrm{~g} / \mathrm{d})$, fresh pork $(12 \cdot 7 \mathrm{~g} / \mathrm{d})$, other red meats such as lamb and horse $(\sim 5 \mathrm{~g} / \mathrm{d})$ and preserved pork and beef $(28 \mathrm{~g} / \mathrm{d}$, corresponding to approximately $40 \mathrm{~g}$ of raw weight). Overall, the estimated consumption of red meat as raw weight was approximately $700 \mathrm{~g}$ /week in the study sample, i.e. significantly higher than the goal.

\section{Choice of descriptive statistics}

Food consumption data are often used to characterize average and high levels of consumption within the population. In the case of food categories which are rarely consumed (either consumed by a limited number of individuals in the population or consumed infrequently by all individuals), due to the large number of non-consumers, very low mean values are obtained and the median can be 0 . It is therefore important to complement the description of food consumption with that of consumption in the selected sample of 'consumers' only.

High levels of consumption were described by providing the 95th and the 99th percentile of the distribution, both in the total population and in consumers only. High percentiles are often needed to estimate dietary exposure to a specific chemical or agent present in food within the process of risk analysis, but caution is needed in the interpretation and use of data due to the short duration of the survey $(3 \mathrm{~d})$. In fact, the observed high percentiles of consumption provide an overestimate of long-term high levels of consumption for many food categories whereas the percentage of consumers provides an underestimate of the long-term percentage of consumers.

The reliability of high percentiles is related to the number of subjects used to calculate them. According to Kroes et al. ${ }^{(24)}$, a high percentile $\mathrm{P}$ (expressed as fraction) can be assessed with sufficient precision if the sample size $n$ satisfies the rule $n(1-\mathrm{P}) \geq 8$. Thus, in Tables $3-11$, high percentiles are reported in parentheses when the number of subjects was lower than 160 (for the 95th percentile) or lower than 800 (for the 99th percentile) to spot the high percentile values which bear a high uncertainty.

\section{Conclusion}

The present paper provides the main results of the Italian National Food Consumption Survey INRAN-SCAI 2005-06 for use both at national and international level. The database obtained from this survey will be the key reference for 
Italian food consumption in the coming years and will be utilized for a variety of purposes including the assessment of nutrient intake and risk analysis.

Some specific aspects of the Italian food consumption pattern are confirmed: a very large contribution from olive oil to fats, a large contribution from wine to alcoholic beverages and a large contribution from bread, pasta and pizza to cereals. The observed high level of consumption of red meat deserves attention.

\section{Acknowledgements}

Sources of funding: The INRAN-SCAI 2005-06 survey was funded by the Italian Ministry of Agriculture, project 'Qualità alimentare'. Conflict of interest declaration: The authors declare that they have no conflicts of interest. Authorship responsibilities: A.T. was the coordinator of INRAN-SCAI 2005-06; C.L. was responsible for the data management system of food diaries and wrote the manuscript; D.A. was responsible for the design of the dietary survey; R.P. was responsible for the management of diaries; S.S. carried out the descriptive data analyses; and C.L.D. developed the dietary assessment tools. All co-authors were involved in the interpretation of results and manuscript revision, and all co-authors approved the final version. The whole INRAN-SCAI 2005-06 Study Group was involved in the preparation of dietary assessment tools, in the training and day-to-day support of field workers, in the data checking and in updating the databases. Acknowledgments: The authors are most thankful to the Italian households who participated to the study and to the field workers: C. Aceto, A. Amoroso, L. Berardini, A. Bertolini, F. Bozzo, M.T. Caprile, E. Cravea, F. Del Greco, N. Donati, P. Gasperoni, R. Gaviglia, T. Gaviglia, E. Giorgeri, R. Ienco, E. Innocenti, U. Margiotta, E. Milesi, S. Mollichelli, E. Moratti, S. Notarnicola, G. Parrino, M. Pasi, R. Pastorini, E. Perrelli, P. Perrucci, C. Sedini, S. Silvestri, F. Simonetti, P. Succi, J. Tabacchi and $\mathrm{P}$. Zaganelli. The authors also express their gratitude to Ager-Agro Ambiente Italia and in particular to G. Massimiliani and E. Sauda for the very positive collaboration. The great competence and availability of $\mathrm{D}$. Berardi (DASC sas) who developed the data management system was highly appreciated. P. Buonocore was helpful in the preparation of the manuscript.

\section{References}

1. Istituto di Servizi per il Mercato Agricolo Alimentare (2007) Consumi Extra Domestici dei prodotti alimentari: Indagine qualitativa II semestre 2006. Rome: ISMEA; available at http://www.ismea.it/flex/cm/pages/ServeBLOB.php/L/IT/ IDPagina/2064

2. Branca F, Nikogosian H \& Lobstein T (editors) (2007) The Challenge of Obesity in the WHO European Region and Strategies for Response. Copenhagen: WHO Regional
Office for Europe; available at http://www.euro.who.int/ document/E90711.pdf

3. Saba A, Turrini A, Mistura G, Cialfa E \& Vichi M (1990) Indagine nazionale sui consumi alimentari delle famiglie 1980-84: alcuni principali risultati (Nation-wide survey on Italian households food consumption 1980-84: main results). J It Soc Food Sci 19, 53-65.

4. Turrini A, Saba A, Perrone D, Cialfa E \& D'Amicis A (2001) Food consumption patterns in Italy: the INN-CA Study 1994-96. Eur J Clin Nutr 55, 571-588.

5. Istituto Nazionale di Statistica (2001) 14 Censimento Generale della Popolazione e delle Abitazioni del 2001. Rome: ISTAT; available at http://dawinci.istat.it/MD/

6. Leclercq C, Berardi D, Sorbillo MR \& Lambe J (1999) Intake of saccharin, aspartame, acesulfame $\mathrm{K}$ and cyclamate in Italian teenagers: present levels and projections. Food Addit Contam 16, 99-109.

7. Leclercq C, Piccinelli R, Arcella D \& Le Donne C (2004) Food consumption and nutrient intake in a sample of Italian secondary school students. Results from the INRANRM-2001 food survey. Int J Food Sci Nutr 55, 265-277.

8. Van Kappel AL, Amoyel J, Slimani N, Vozar B \& Riboli E (1994) EPIC-SOFT Picture Book For Estimating Portion Sizes. Lyon: IARC Press.

9. Le Donne C, Arcella D, Piccinelli R, Sette S, Berardi D, Leclercq C (2006) Benefits of the use of a multi-operator data management system for the collection of consumption data through food diaries. Sixth International Conference on Dietary Assessment Methods, Copenhagen, 27-29 April 2006. Book of Abstracts, p. 121, P12-07. http://www. icdam6.dk/filer/Abstracts.pdf (accessed July 2008).

10. Commission of the European Communities (1993) Nutrient and Energy Intakes for the European Community (opinion expressed on 11 December 1992). Report of the Scientific Committee for Food Thirty-first series. Luxembourg: Office for Official Publications of the European Communities; available at http://ec.europa.eu/food/fs/sc/scf/out89.pdf.

11. European Food Safety Authority (2005) Opinion of the Scientific Committee on a request from EFSA related to Exposure Assessments (adopted on 22 June 2005). The EFSA Journal 249, 1-26; available at http://www.efsa. europa.eu/EFSA/efsa_locale-1178620753812_1178620763345. htm

12. Istituto Nazionale di Statistica (2007) Le imprese di telecomunicazioni Anno 2005. Rome: ISTAT; available at http://www.istat.it/salastampa/comunicati/non_calendario/ 20070214_00/

13. Verger P, Ireland J, Møller A, Abravicius JA, De Henauw S \& Naska A for the EFCOSUM Group (2002) Improvement of comparability of dietary intake assessment using currently available individual food consumption surveys. Eur J Clin Nutr 56, Suppl. 2, S18-S24.

14. Kiely M, Flynn A, Harrington KE, Robson PJ \& Cran G (2001) Sampling description and procedures used to conduct the North/South Ireland Food Consumption Survey. Public Health Nutr 4, 1029-1035.

15. Ocké MC, Hulshof KFAM \& van Rossum CTM (2005) The Dutch national food consumption survey 2003. Methodological issues. Arch Public Health 63, 227-241.

16. Hoare J, Henderson L, Bates CJ, Prentice A, Birch M, Swan G \& Farron M (2004) The National Diet and Nutrition Survey: Adults Aged 19 to 64 Years. Summary Report. London: TSO; available at http://www.foodstandards. gov.uk/multimedia/pdfs/ndns5full.pdf

17. Istituto Nazionale di Statistica (2006) Bilancio demografico anno 2006 e popolazione residente al 31 dicembre. Rome: ISTAT; available at http://demo.istat.it/pop2006/ index.html

18. Istituto Nazionale di Statistica (2005) L'istruzione della popolazione al 2001. Dati definitivi del censimento. Rome: ISTAT; 
available at http://www.istat.it/salastampa/comunicati/non_ calendario/20050121_00/testointegrale.pdf

19. Goldberg GR, Black AE, Jebb SA, Cole TJ, Murgatroyd PR, Coward WA \& Prentice AM (1991) Critical evaluation of energy intake data using fundamental principles of energy physiology: 1 . Derivation of cut-off limits to identify underrecording. Eur J Clin Nutr 45, 569-581.

20. McGowan MJ, Harrington KE, Kiely M, Robson PJ, Livingstone MBE \& Gibney MJ (2001) An evaluation of energy intakes and the ratio of energy intake to estimated basal metabolic rate $\left(\mathrm{EI} / \mathrm{BMR}_{\mathrm{est}}\right)$ in the North/South Ireland Food Consumption Survey. Public Health Nutr 4, 1043-1050.

21. Istituto di Servizi per il Mercato Agricolo Alimentare (2005) Consumi - Indagini speciali. Acquisti domestici: oli e grassi. La tendenza degli acquisti in volume 2000-2004 http://www.ismea.it/flex/cm/pages/ServeBLOB.php/L/IT/ IDPagina/1266 (accessed July 2008).

22. Food and Agriculture Organization of the United Nations/ World Health Organization (2002) Diet, Nutrition and the Prevention of Chronic Diseases. Joint WHO/FAO Expert Consultation. WHO Technical Report Series no. 916. Geneva: WHO.

23. World Cancer Research Fund/American Institute for Cancer Research (2007) Food, Nutrition, Physical Activity, and the Prevention of Cancer: A Global Perspective. Washington, DC: AICR.

24. Kroes R, Müller D, Lambe J et al. (2002) Assessment of intake from the diet. Food Chem Toxicol 40, 327-385.

\section{Appendix}

Food items included in food categories: Italian National Food Consumption Survey INRAN-SCAI 2005-06

Most frequent minor ingredients from other food categories present in composite foods

\begin{tabular}{|c|c|}
\hline Food categories & $\begin{array}{l}\text { List of single food items and of composite food items } \\
\text { classified in the food category }\end{array}$ \\
\hline \multicolumn{2}{|l|}{$\begin{array}{l}\text { Cereals, cereal products and } \\
\text { substitutes }\end{array}$} \\
\hline Bread & $\begin{array}{l}\text { All types of bread prepared with any type of flour } \\
\text { (wheat, whole wheat, soya, maize, etc.), speciality } \\
\text { breads, sponge bread, excl. breadcrumbs and } \\
\text { toasted bread }\end{array}$ \\
\hline Pasta and pasta substitutes & $\begin{array}{l}\text { All types of pasta, incl. rice noodles, fresh pasta with } \\
\text { eggs, tortellini (fresh pasta with meat filling) and } \\
\text { pasta for infants, puff pastry dough }\end{array}$ \\
\hline Pizza & $\begin{array}{l}\text { Plain white pizza, plain tomato pizza, excl. other pizzas } \\
\text { which were codified through recipes }\end{array}$ \\
\hline Rice & $\begin{array}{l}\text { All types of rice, white and brown, excl. rice in } \\
\text { commercial rice pudding }\end{array}$ \\
\hline $\begin{array}{l}\text { Wheat, other cereals } \\
\text { and flours }\end{array}$ & $\begin{array}{l}\text { All types of flour (wheat, corn, oat, couscous, millet, } \\
\text { barley, rice, etc.) used in recipes (polenta, pizza, } \\
\text { fresh pasta, cakes), breadcrumbs, baby cereals }\end{array}$ \\
\hline Breakfast cereals & $\begin{array}{l}\text { All types of ready-to-eat cereals (generally used at } \\
\text { breakfast with milk): corn flakes, puffed wheat, dried } \\
\text { and toasted rice, muesli, etc. }\end{array}$ \\
\hline Biscuits & $\begin{array}{l}\text { All types of sweet biscuits, incl. sugar-free biscuits, } \\
\text { granulated biscuits for infants, gluten-free biscuits }\end{array}$ \\
\hline $\begin{array}{l}\text { Savoury fine bakery } \\
\text { products }\end{array}$ & $\begin{array}{l}\text { All types of crackers, breadsticks, rusk, popcorn, } \\
\text { salty appetizers and savoury biscuits }\end{array}$ \\
\hline Cakes and sweet snacks & $\begin{array}{l}\text { Sweet rolls, all types of cakes (plain, with chocolate, } \\
\text { with fruit, with cream, etc.), sweet cereal-based } \\
\text { snacks (e.g. bars) }\end{array}$ \\
\hline Pulses, fresh and processed & $\begin{array}{l}\text { All types of pulses (fresh and processed): lentils, peas, } \\
\text { chickpeas, all types of beans excl. green beans, } \\
\text { soyabeans }\end{array}$ \\
\hline \multicolumn{2}{|l|}{$\begin{array}{l}\text { Vegetables, fresh and } \\
\text { processed }\end{array}$} \\
\hline Leafy vegetables, fresh & Chicory, lettuce, spinach, etc. \\
\hline Tomatoes, fresh & $\begin{array}{l}\text { Fresh tomatoes used in salad and other recipes (e.g. } \\
\text { tomato sauce) }\end{array}$ \\
\hline $\begin{array}{l}\text { Other fruiting vegetables, } \\
\text { fresh }\end{array}$ & Aubergine, pepper, cucumber, pumpkin, courgette, etc \\
\hline Roots and onions, fresh & Onion, garlic, turnip-rooted celery, beetroot, daikon \\
\hline Other vegetables, fresh & $\begin{array}{l}\text { All other types of fresh vegetables: broccoli, cabbage, } \\
\text { cauliflower, artichokes, asparagus, fennel, } \\
\text { mushroom, green beans and palm heart }\end{array}$ \\
\hline Vegetables, processed & $\begin{array}{l}\text { Vegetable preserves (in vinegar, oil or brine), canned } \\
\text { tomatoes, vegetable soup dehydrated, industrial } \\
\text { mixed salad with mayonnaise, homogenized } \\
\text { vegetables for infants }\end{array}$ \\
\hline Spices and herbs & $\begin{array}{l}\text { Parsley, basil, rosemary, oregano, sesame seed, } \\
\text { curry, ginseng, etc. }\end{array}$ \\
\hline
\end{tabular}

Cereals, cereal products and substitutes

Bread

All types of bread prepared with any type of flour breads, sponge bread, excl. breadcrumbs and pastry dough

lain white pizza, plain tomato pizza, excl. other pizzas which were codified through recipes

commercial rice pudding

types of flour (wheat, corn, oat, couscous, millet, ): corn flakes, puffed wheat, dried and toasted rice, muesli, etc. All types of crackers, breadsticks, rusk, popcorn, salty appetizers and savoury biscuits , with cream, etc), sweet cereal-based All types of pulses (fresh and processed): lentils, peas, chickpeas, all types of beans excl. green beans, soyabeans

Chicory, lettuce, spinach, etc.

tomato sauce)

Onion, garlic, turnip-rooted celery, beetroot, daikon cauliflower, artichokes, asparagus, fennel, mushroom, green beans and palm heart getable preserves (in vinegar, oil or brine), canned vegetables for infants curry, ginseng, etc.

Milk and fats in speciality breads; soya in soya bread

Meat in tortellini; eggs in fresh pasta; butter in puff pastry dough

Tomato in plain tomato pizza; oil in plain white pizza and plain tomato pizza

Fruit in some baby cereals

Dried fruit and nuts in muesli; sugar; cocoa

Sugar; eggs; fats; cocoa; dried fruit and nuts

Fats

Fats; eggs; fruit; milk

$-$

$-$

Eggs (in mayonnaise); oil (in oil preserves and mayonnaise); cereals and potatoes (in dehydrated soup); vinegar (in oil preserves) 
Appendix Continued

\begin{tabular}{|c|c|}
\hline Food categories & $\begin{array}{l}\text { List of single food items and of composite food items } \\
\text { classified in the food category }\end{array}$ \\
\hline $\begin{array}{l}\text { Potatoes, tubers and their } \\
\text { products }\end{array}$ & $\begin{array}{l}\text { Potatoes raw, sweet potatoes, tapioca, potato chips, } \\
\text { potato chips with cheese, potato croquettes, potato } \\
\text { flakes dehydrated }\end{array}$ \\
\hline \multicolumn{2}{|l|}{ Fruit, fresh and processed } \\
\hline Citrus fruit, fresh & Orange, lemon, grapefruit, tangerine, tangelo \\
\hline Exotic fruit, fresh & Banana, cherimoya, avocado, papaya, mango, lychee \\
\hline Other fruit, fresh & $\begin{array}{l}\text { All other types of fruit: apple, pear, peach, apricot, } \\
\text { grape, fig, melon, watermelon, strawberry, } \\
\text { raspberry, pomegranate, plum, cherry, etc. }\end{array}$ \\
\hline $\begin{array}{l}\text { Nuts, seeds, olives and } \\
\text { their products, dried fruit }\end{array}$ & $\begin{array}{l}\text { Nuts roasted, dried, in powder or in purée (almond, } \\
\text { chestnut, walnut, coconut, pine nut, peanut, } \\
\text { pistachio), seeds (pumpkin seed) and all dried fruit } \\
\text { (figs, plum, raisin), olives }\end{array}$ \\
\hline $\begin{array}{l}\text { Other processed fruit } \\
\quad \text { (in syrup, in purée, etc.) }\end{array}$ & $\begin{array}{l}\text { All types of fruit in syrup (peach, apricot, pear, fruit } \\
\text { cocktail. etc.), in syrup, fruit purée, minced fruit, incl. } \\
\text { homogenized fruit for infants }\end{array}$ \\
\hline
\end{tabular}

Most frequent minor ingredients from other food categories present in composite foods

Fats in all potato chips; cheese in some speciality potato chips; breading in potato croquettes

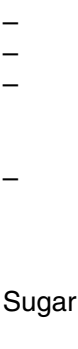

Meat, meat products and substitutes

Beef and veal, not preserved, excl. offal

Pork, not preserved, excl. offal

Poultry and game, not preserved, excl. offal

Other meats, not preserved excl. offal

Beef, veal, homogenized meat for infants, industrial meat sauce

Pork meat, pork meat roasted (porchetta), foot pork raw, excl. offals

Pheasant, chicken, roast chicken, goose, quail, turkey, ostrich, incl. homogenized products for infants

Lamb, goat, mutton, kid, horse, donkey, lean deer, boar, rabbit, frog, land snail, incl. homogenized products for infants, excl. offals

Ham, salami, sausages and Preserved meat from pork, chicken and turkey meat other preserved meats, excl. offal (ham, mortadella, sausages, wurstel, salami, etc.), roast beef, canned beef, dried beef or horse meat (bresaola), incl. homogenized products for infants

Offal, blood and their products

Meat substitutes

Liver, kidney, brain, trotter, heart, tripe, tongue, sweetbreads, blood and liver pâté

Seitan (wheat gluten), soya hamburger

Fish, seafood and their products

Fish and seafood, fresh and frozen

All types of fish, molluscs, crustaceans, raw (fresh or frozen) and fish fingers

Fish and seafood, preserved All types of preserved fish, molluscs, crustaceans and fish eggs (caviar, anchovies brined or in oil, tuna brined or in oil, smoked salmon, canned crab meat, cod dried and salted, smoked herring, etc.) incl. homogenized products for infants

Milk, milk products and

substitutes

Milk, milk-based beverages, All types of milk (liquid, condensed and powder form), human milk, infant formula and substitutes incl. infant formulae, human milk and milk substitutes (e.g. soya, rice, oat), flavoured milk (e.g. packaged chocolate-flavoured milk)

Yoghurt and fermented milk Yoghurt, drinkable yoghurt, yoghurt-based dessert with fruit for infants

Cheese and substitutes

All types of cheese (e.g. mozzarella, parmesan, edam, feta, fontina), flavoured sweet cheese for children, cheese substitutes (e.g. tofu), homogenized cheese products for infants

Milk-based desserts and substitutes

Commercial milk-based desserts (e.g. mousse chocolate, custard, chantilly), incl. commercial creamy rice pudding (risolatte)

Oils and fats

Olive oil

Other vegetable oils

Butter and creams

Other fats
Tomato in industrial meat sauce; fats, cereals and tubers in homogenized baby food

Fats, cereals and tubers in homogenized baby food

Fats, cereals and tubers in homogenized baby food

Fats, cereals and tubers in homogenized baby food

Fats in liver pâté

Soya, wheat

Breading on fish fingers

Oil in oil preserves

Sugar in flavoured milk beverages; cocoa in flavoured milk beverages; soya, rice in milk substitutes

Sugar in yoghurt; fruit or cereals in yoghurt

Fruit and sugar in flavoured cheese for children

Sugar; milk cream; cocoa; rice in rice pudding

$-$

Eggs in mayonnaise
Extra-virgin olive oil, olive oil, incl. enriched type

All type of vegetable oils, excl. olive oil

Butter, cream, heavy cream

Margarine all types, mayonnaise, lard, incl. soyabean butter 
Appendix Continued

\begin{tabular}{|c|c|c|}
\hline Food categories & $\begin{array}{l}\text { List of single food items and of composite food items } \\
\text { classified in the food category }\end{array}$ & $\begin{array}{l}\text { Most frequent minor ingredients from other food } \\
\text { categories present in composite foods }\end{array}$ \\
\hline Eggs & $\begin{array}{l}\text { All types of eggs (chicken, duck, ostrich, etc.) } \\
\text { excl. fish eggs }\end{array}$ & - \\
\hline \multicolumn{3}{|l|}{$\begin{array}{l}\text { Alcoholic beverages } \\
\text { and substitutes }\end{array}$} \\
\hline $\begin{array}{l}\text { Regular wine and } \\
\text { substitutes }\end{array}$ & $\begin{array}{l}\text { All types of wine (red, white), 'non-alcoholic' } \\
\text { wine, excl. sparkling wine }\end{array}$ & - \\
\hline Beer, cider and substitutes & Beer all types, non-alcoholic beer & - \\
\hline $\begin{array}{l}\text { Sweet wine, spumante, } \\
\text { wine-based appetizers, } \\
\text { spirits and liquors }\end{array}$ & $\begin{array}{l}\text { Sparkling wine, fortified wine (e.g. porto, cherry, } \\
\text { vermouth, dessert wine sweet or dry, appetizer), } \\
\text { spirits (e.g. grappa, brandy, whiskey), liquors } \\
\text { (amaro, limoncello, etc.) }\end{array}$ & - \\
\hline \multicolumn{3}{|l|}{$\begin{array}{l}\text { Sweet products and } \\
\text { substitutes }\end{array}$} \\
\hline $\begin{array}{l}\text { Ice cream, ice lolly and } \\
\text { substitutes }\end{array}$ & $\begin{array}{l}\text { All types of ice creams (with all possible flavours } \\
\text { and ingredients), ice lolly }\end{array}$ & $\begin{array}{l}\text { Milk in ice cream; soya in soya ice cream; fruit; } \\
\text { eggs }\end{array}$ \\
\hline Chocolate and substitutes & $\begin{array}{l}\text { All types of chocolate (milk, white, cocoa } 70-90 \% \text {, with } \\
\text { hazelnuts, etc.), spreadable chocolate cream with } \\
\text { or without hazelnuts, chocolate bars filled with mou }\end{array}$ & $\begin{array}{l}\text { Milk in milk chocolate; nuts in some chocolate } \\
\text { creams or bars; rice in some chocolate bars }\end{array}$ \\
\hline $\begin{array}{l}\text { Candies, jam and other } \\
\text { sweet products (incl. } \\
\text { sugar-free) }\end{array}$ & $\begin{array}{l}\text { All types of candies (e.g. mou, fudge, fondant, different } \\
\text { flavours), chewing gum, jam, marmalade, nougat } \\
\text { with almonds, glazed chestnuts (marrons glacés), } \\
\text { peanut brittle, sesame brittle, incl. sugar-free } \\
\text { products }\end{array}$ & Nuts; seeds \\
\hline $\begin{array}{l}\text { Sugar, fructose, honey and } \\
\text { other nutritious sweeteners }\end{array}$ & Honey, sugar, fructose, maple syrup, royal jelly & - \\
\hline $\begin{array}{l}\text { Cocoa and cocoa-based } \\
\text { powder }\end{array}$ & $\begin{array}{l}\text { Cocoa powder, mixed powder of cocoa with other } \\
\text { ingredients, with or without sugar, with or without } \\
\text { dehydrated milk }\end{array}$ & Sugar; milk \\
\hline Artificial sweeteners & $\begin{array}{l}\text { All types of table-top products containing artificial } \\
\text { sweeteners (e.g. aspartame, saccharin) in tablets, } \\
\text { powder or liquid }\end{array}$ & - \\
\hline Meal substitutes & Meal replacements in liquid form and bar form & - \\
\hline \multicolumn{3}{|l|}{$\begin{array}{l}\text { Water and other non-alcoholic } \\
\text { beverages }\end{array}$} \\
\hline $\begin{array}{l}\text { Tap water (as such, in } \\
\text { beverages or recipes) }\end{array}$ & Tap water & - \\
\hline Bottled water & All types of commercial bottled water & - \\
\hline $\begin{array}{l}\text { Coffee, tea, herbal tea and } \\
\text { substitutes }\end{array}$ & $\begin{array}{l}\text { All types of coffee (e.g. brewed, decaffeinated, } \\
\text { 'espresso', powdered), all types of tea (powdered, } \\
\text { brewed, deteinated) with or without sugar, herbal } \\
\text { tea (e.g. chamomille), pearled barley coffee }\end{array}$ & $\begin{array}{l}\text { Sugar in powders for the preparation of instant } \\
\text { beverages }\end{array}$ \\
\hline Fruit and vegetable juices & $\begin{array}{l}\text { All types of fruit and vegetable juices (e.g. nectar, } \\
\text { carrot juice, orange juice), with or without the } \\
\text { addition of water, sugar and intense sweeteners, } \\
\text { incl. fortified products }\end{array}$ & - \\
\hline Other soft drinks & $\begin{array}{l}\text { All types of carbonate beverages (e.g. cola, soda, } \\
\text { ginger ale, orange, tonic water) with sugar or sugar- } \\
\text { free, ice tea, ice herbal tea, energy drinks, sport } \\
\text { drinks, syrups to be diluted (incl. almond milk) }\end{array}$ & - \\
\hline Miscellaneous & $\begin{array}{l}\text { Vinegar, ketchup, mustard sauce, meat cubes, bouillon } \\
\text { cubes, yeast, leavening agents, soya sauce, sodium } \\
\text { bicarbonate (used for household production of fizzy } \\
\text { water from tap water) }\end{array}$ & $\begin{array}{l}\text { Tomato and sugar in ketchup; meat in meat } \\
\text { cubes }\end{array}$ \\
\hline Liquid foods & $\begin{array}{l}\text { All water and beverages, milk in liquid state, drinkable } \\
\text { yoghurt }\end{array}$ & \\
\hline Solid foods & $\begin{array}{l}\text { All other food items incl. oil, dehydrated items (milk), } \\
\text { powder for reconstitution of beverages, creamy } \\
\text { desserts (yoghurt), sauces, etc. }\end{array}$ & \\
\hline
\end{tabular}

